\title{
ON STABILITY OF STANDING WAVES OF NONLINEAR DIRAC EQUATIONS
}

\author{
NABILE BOUSSAID AND SCIPIO CUCCAGNA
}

\begin{abstract}
We consider the stability problem for standing waves of nonlinear Dirac models. Under a suitable definition of linear stability, and under some restriction on the spectrum, we prove at the same time orbital and asymptotic stability. We are not able to get the full result proved in [26] for the nonlinear Schrödinger equation, because of the strong indefiniteness of the energy.
\end{abstract}

\section{INTRODUCTION}

In this paper we study the stability of standing waves of a class of nonlinear Dirac equations (NLDE). We assume that these standing waves are smooth, have exponential decay to 0 at infinity and that they are smoothly dependent on a parameter. We then partially characterize, under a number of further technical hypotheses, their stability and their instability. We succeed partially in transposing to NLDE results proved for the nonlinear Schrödinger equations (NLS) in [26] and in previous references. We recall that $15,49,50,61,62,35,36$ contain a quite satisfactory characterization of the orbital stability of standing waves of the NLS. They do not apply to the Dirac equation, due to the strong indefiniteness of the energy. In this paper we initiate a theory of stability in the case of the NLDE, using ideas coming from the theory of asymptotic stability which are less sensitive to indefiniteness of the energy. This idea is explored also in [46] in a very special situation.

1.1. The nonlinear Dirac equation. We consider for $m>0$ a NLDE

$$
\left\{\begin{array}{c}
\mathrm{i} u_{t}-D_{m} u+g(u \bar{u}) \beta u=0 \\
u(0, x)=u_{0}(x)
\end{array}(t, x) \in \mathbb{R} \times \mathbb{R}^{3}\right.
$$

where $D_{m}=-\mathrm{i} \sum_{j=1}^{3} \alpha_{j} \partial_{x_{j}}+m \beta$, with for $j=1,2,3$

$$
\alpha_{j}=\left(\begin{array}{cc}
0 & \sigma_{j} \\
\sigma_{j} & 0
\end{array}\right), \quad \beta=\left(\begin{array}{cc}
I_{\mathbb{C}^{2}} & 0 \\
0 & -I_{\mathbb{C}^{2}}
\end{array}\right), \quad \sigma_{1}=\left(\begin{array}{cc}
0 & 1 \\
1 & 0
\end{array}\right), \sigma_{2}=\left(\begin{array}{cc}
0 & \mathrm{i} \\
-\mathrm{i} & 0
\end{array}\right), \sigma_{3}=\left(\begin{array}{cc}
1 & 0 \\
0 & -1
\end{array}\right) .
$$

The unknown $u$ is $\mathbb{C}^{4}$-valued. Given two vectors of $\mathbb{C}^{4}, u v:=u \cdot v$ is the inner product in $\mathbb{C}^{4}, v^{*}$ is the complex conjugate, $u \cdot v^{*}$ is the hermitian product in $\mathbb{C}^{4}$, which we write as $u v^{*}=u \cdot v^{*}$. We set $\bar{u}:=\beta u^{*}$, so that $u \bar{u}=u \cdot \beta u^{*}$. We have

$$
\alpha_{j} \alpha_{\ell}+\alpha_{\ell} \alpha_{j}=2 \delta_{j \ell} I_{\mathbb{C}^{4}}, \quad \alpha_{j} \beta+\beta \alpha_{j}=0, \quad \beta^{2}=I_{\mathbb{C}^{4}} .
$$

Thus the operator $D_{m}$ is self-adjoint on $L^{2}\left(\mathbb{R}^{3}, \mathbb{C}^{4}\right)$, with domain $H^{1}\left(\mathbb{R}^{3}, \mathbb{C}^{4}\right)$ and we have $D_{m}^{2}=-\Delta+m^{2}$. The spectrum is $\sigma\left(D_{m}\right)=(-\infty,-m] \cup[m,+\infty)$, see [59, Theorem 1.1].

1.2. State of the art. The equation in $\$ 1.1$ arises in Dirac models used to model either extended particles with self-interaction or particles in space-time with geometrical structure. In the latter case, physicists have shown that a relativistic theory sometimes imposes a fourth order nonlinear potential (i.e., a cubic nonlinearity) such as the square of a quadratic form on $\mathbb{C}^{4}$; see [4] and the references therein. The associated stationary equation is called the Soler model, 52, as it was proposed by Soler to model the elementary fermions.

In our study, we assume the existence of stationary solutions as well as a number of properties like the smooth dependence on a parameter, the smoothness and the fact that they are rapidly decaying. These are not well established properties. Stationary solutions were actively studied in the last thirty years. References 16, 42, 3, 4 used a dynamical systems approach. For the use of the variational structure of the stationary equation, see [32. For an approach yielding stationary solutions of the NLDE from solutions of the NLS, see [45, 37.

Turning to the question of stability, [55] discusses the Soler model within the framework of [50], without attempting a proof. Some partial results involving small standing waves obtained by bifurcation from linear ones, with $D_{m}$ replaced by $H:=D_{m}+V$ with $V$ a nice potential, are in [9, 10. 10] shows that if a resonance condition holds, there is a stable manifold outside which any initial condition leads 
to instability. If the resonance condition is not fulfilled, the stability problem is left open. The results we present here answer this question and can be used to clarify 9. 41] proves the existence of global attractors in a model involving a Dirac equation coupled to an harmonic oscillator. The stability problem for the 1 dimensional NLDE is discussed under very restrictive hypotheses in [46 which reproduces for the $1 \mathrm{D}$ NLDE an analogue of the result in 57 .

1.3. Hypotheses. We assume the following hypotheses (H:1) (H:12).

(H:1) $g(0)=0, g \in C^{\infty}(\mathbb{R}, \mathbb{R})$.

(H:2) There exists an open interval $\mathcal{O} \subseteq(m / 3, m)$ such that $D_{m} u-\omega u-g(u \bar{u}) \beta u=0$ admits a $C^{\infty}$ family of solutions $\omega \in \mathcal{O} \rightarrow \phi_{\omega} \in H^{k, \tau}\left(\mathbb{R}^{3}\right)$ for any $(k, \tau)$, see (1.4) for a definition. In spherical coordinates $x_{1}=\rho \cos (\vartheta) \sin (\varphi), x_{2}=\rho \sin (\vartheta) \sin (\varphi), x_{3}=\rho \cos (\varphi)$, these standing waves are of the form

$$
\phi_{\omega}(x)=\left[\begin{array}{c}
a(\rho)\left[\begin{array}{l}
1 \\
0
\end{array}\right] \\
\mathrm{i} b(\rho)\left[\begin{array}{c}
\cos \varphi \\
e^{\mathrm{i} \vartheta} \sin \varphi
\end{array}\right]
\end{array}\right]
$$

with $a(\rho)$ and $b(\rho)$ real valued and satisfying the following properties:

$$
\begin{aligned}
& a, b \in C^{\infty}([0, \infty), \mathbb{R}), \quad \forall \rho \geq 0, \quad a^{2}(\rho)-b^{2}(\rho) \geq 0, \\
& a^{(j)} \text { and } b^{(j)} \text { decay exponentially at infinity for all } j .
\end{aligned}
$$

Notice that $\phi_{\omega}(-x)=\beta \phi_{\omega}(x)$ and $\phi_{\omega}\left(-x_{1},-x_{2}, x_{3}\right)=S_{3} \phi_{\omega}\left(x_{1}, x_{2}, x_{3}\right)$ with $S_{3}:=\left(\begin{array}{cc}\sigma_{3} & 0 \\ 0 & \sigma_{3}\end{array}\right)$.

(H:3) Let $q(\omega)=\left\|\phi_{\omega}\right\|_{L^{2}}^{2}$. We assume $q^{\prime}(\omega) \neq 0$ for all $\omega \in \mathcal{O}$.

(H:4) For any $x \in \mathbb{R}^{3}$ we consider in (1.1) initial data s.t. $u_{0}(-x)=\beta u_{0}(x)$ and $u_{0}\left(-x_{1},-x_{2}, x_{3}\right)=$ $S_{3} u_{0}\left(x_{1}, x_{2}, x_{3}\right)$.

(H:5) Let $\mathcal{H}_{\omega}$ be the linearized operator around $e^{\mathrm{i} t \omega} \phi_{\omega}$, see Sect. 3. We assume that $\mathcal{H}_{\omega}$ satisfies the definition of linear stability in Definition 3.4.

(H:6) Consider $\mathbf{X}:=\left\{\left(\Upsilon_{1}, \Upsilon_{2}\right) \in L^{2}\left(\mathbb{R}^{3},\left(\mathbb{C}^{4}\right)^{2}\right):\left(\Upsilon_{1}(-x), \Upsilon_{2}\left(-x_{1},-x_{2}, x_{3}\right) \equiv\left(\beta \Upsilon_{1}(x),-\beta \Upsilon_{1}(x)\right.\right.\right.$, $\left(\Upsilon_{1}\left(-x_{1},-x_{2}, x_{3}\right), \Upsilon_{2}\left(-x_{1},-x_{2}, x_{3}\right) \equiv\left(S_{3} \Upsilon_{1}(x),-S_{3} \Upsilon_{1}(x)\right)\right\}$, see Sect. 3 and under Lemma 2.3. $\mathbf{X}$ is invariant for the action of $\mathcal{H}_{\omega}$. Consider the restriction of $\mathcal{H}_{\omega}$ in $\mathbf{X}$. Then $\mathcal{H}_{\omega}$ has $2 n$ nonzero eigenvalues, counted with multiplicity, all contained in $(\omega-m, m-\omega)$. The positive eigenvalues can be listed as $0<\lambda_{1}(\omega) \leq \ldots \leq \lambda_{n}(\omega)<m-\omega$, where we repeat each eigenvalue according to the multiplicity. For each $\lambda_{j}(\omega)$, also $-\lambda_{j}(\omega)$ is an eigenvalue (this symmetry follows from (2.9) $)$. There are no other eigenvalues except for 0 .

(H:7) The points and $\pm(m-\omega)$ and $\pm(m+\omega)$ are not resonances for $\mathcal{H}_{\omega}$, see (1.2)- (1.3) below.

(H:8) Suppose that $\lambda \in \mathbb{R}$ with $|\lambda|>m-\omega$ is a resonance for $\mathcal{H}_{\omega}$, that is one of the following two equations admits a nontrivial solution:

$$
\begin{aligned}
& \left(1+R_{\mathcal{H}_{\omega, 0}}^{+}(\lambda) V_{\omega}\right) u=0, \quad u \in L^{2,-\tau}\left(\mathbb{R}^{3}, \mathbb{C}^{8}\right) \text { for some } \tau>1 / 2 ; \\
& \left(1+R_{\mathcal{H}_{\omega, 0}}^{-}(\lambda) V_{\omega}\right) u=0, \quad u \in L^{2,-\tau}\left(\mathbb{R}^{3}, \mathbb{C}^{8}\right) \text { for some } \tau>1 / 2 .
\end{aligned}
$$

Then if $u$ satisfies either (1.2) or (1.3) we have $u \in L^{2}\left(\mathbb{R}^{3}, \mathbb{C}^{8}\right)$ and $\lambda$ is an eigenvalue of $\mathcal{H}_{\omega}$.

(H:9) There are natural numbers $N_{j}$ defined by the property $0<N_{j} \lambda_{j}(\omega)<m-\omega<\left(N_{j}+1\right) \lambda_{j}(\omega)$.

(H:10) There is no multi index $\mu \in \mathbb{Z}^{k}$ with $|\mu|:=\left|\mu_{1}\right|+\ldots+\left|\mu_{k}\right| \leq 2 N_{1}+3$ such that $\mu \cdot \lambda=m \pm \omega$.

(H:11) If $\lambda_{j_{1}}<\ldots<\lambda_{j_{k}}$ are $k$ distinct $\lambda$ 's, and $\mu \in \mathbb{Z}^{k}$ satisfies $|\mu| \leq 2 N_{1}+3$, then we have

$$
\mu_{1} \lambda_{j_{1}}+\cdots+\mu_{k} \lambda_{j_{k}}=0 \Longleftrightarrow \mu=0 .
$$

(H:12) The nonlinear Fermi golden rule (11.12) is true.

The space of functions satisfying (H:4) is invariant by (1.1). Except for the smoothness with respect to the parameter $\omega$, for some non-linearities (H:2) is a consequence of [32]. Continuous dependence on $\omega$ for some examples is proved in $[37$.

Remark 1.1. $2 \omega$ is always an eigenvalue of $\mathcal{H}_{\omega}$ in $L^{2}\left(\mathbb{R}^{3}, \mathbb{C}^{8}\right),[20$. So for $3 \omega>m$ we have $2 \omega \in$ $(m-\omega, m+\omega)$ is an embedded eigenvalue. We can avoid it thanks to the symmetry (H:4) since the eigenvectors do not belong to $\mathbf{X}$, see Lemma 3.1 below and subsequent comments. Reducing to the space $\mathbf{X}$ reduces the number of parameters, simplifying the problem. The parameters eliminated involve translation and orientation of the solutions. For work on moving ground states of the NLS see [27]. 
Remark 1.2. By $(\mathbf{H : 6})(\mathbf{H : 8})$ there are no resonances for the restriction of $\mathcal{H}_{\omega}$ in $\mathbf{X}$. $(\mathbf{H : 8})$ is proved in the case of the NLS assumption in [29]. In the case of the Dirac system we are not able to prove it except for resonances contained in $(-\omega+m, \omega-m)$ or for large energies. This is yet a consequence of the strong indefiniteness of the energy of the Dirac system. We expect that $(\mathbf{H : 8})$ can be eliminated.

1.4. Main results. The main result in this article is the following one.

Theorem 1.3. Suppose that $\mathcal{O} \subset(m / 3, m)$ and fix $k_{0} \geq 4, k_{0} \in \mathbb{Z}$. Pick $\omega_{1} \in \mathcal{O}$ and let $\phi_{\omega_{1}}(x)$ be a standing wave of (1.1). Let $u(t, x)$ be a solution to (1.1). Assume $(\mathbf{H : 1 )}(\mathbf{H : 1 2})$, Then, there exist an $\epsilon_{0}>0$ and $a C>0$ such that for any $\epsilon \in\left(0, \epsilon_{0}\right)$ and for any $u_{0}$ with $\inf _{\gamma \in \mathbb{R}}\left\|u_{0}-e^{\mathrm{i} \gamma} \phi_{\omega_{1}}\right\|_{H^{k_{0}}}<\epsilon$, there exist $\omega_{+} \in \mathcal{O}, \theta \in C^{1}(\mathbb{R} ; \mathbb{R})$ and $h_{+} \in H^{k_{0}}$ with $\left\|h_{+}\right\|_{H^{k_{0}}}+\left|\omega_{+}-\omega_{1}\right| \leq C \epsilon$ such that

$$
\lim _{t \rightarrow+\infty}\left\|u(t, \cdot)-e^{\mathrm{i} \theta(t)} \phi_{\omega_{+}}-e^{-\mathrm{i} t D_{m}} h_{+}\right\|_{H^{k_{0}}}=0 .
$$

Remark 1.4. The constraint $3 \omega>m$ allows to exploit the nonlinear Fermi Golden Rule (FGR) like for the NLS in 26] by circumventing the strong indefiniteness of the Dirac system. We expect that that the hypothesis $3 \omega>m$ can be eliminated. Specifically, it is used to guarantee that appropriate multiples of the eigenvalues belong to portions of the spectrum where there is no superposition of the continuous spectrum of distinct coordinates. This fact and our results continue to hold if $3 \omega<m$ and $\left(2 N_{j}+1\right) \omega>m$ for all $j=1, \ldots, n$, see Remark 11.3 .

Remark 1.5. Energy indefiniteness affects our methods because it results in superposition of the continuous spectrum of distinct coordinates. There are two points where our methods are affected. The first is discussed in Remark 1.4 The second point is when we take $(\mathbf{H : 8 )}$ as an hypothesis, see Remark 1.2 .

Remark 1.6. We do not know of examples of $g$ and $\omega$ satisfying our spectral assumptions. The situation is not very different from the case of the NLS where the spectrum is unknown except in few cases. Rigorous analysis of examples is certainly a difficult open problem. Like for the NLS, see [17, one can consider numerical analysis. For some example in 1-D see [6, 19. For NLDE, by a non relativistic bifurcation argument, see [45, 37, 31, it is possible to extend what is known for the NLS equation to the NLDE for $\omega$ close to $m$.

Remark 1.7. A partial justification of our hypotheses can be given using bifurcation theory from linear problem, see [9, 10, 46, 56, 57]. It is easy to prove the existence of "small solitons" for which (H:1) (H:11) hold. In this context the symmetry $\phi_{\omega}(-x)=\beta \phi_{\omega}(x)$ and in (H:4) are unnecessary. In particular (H:6) holds replacing $\mathbf{X}$ with $L^{2}\left(\mathbb{R}^{3}, \mathbb{C}^{8}\right)$. In the set up of small solitons, $(\mathbf{H : 6})$ and $(\mathbf{H : 8})$ are always true while (H:7) and (H:9) (H:11) hold generically. In the context of small solitons it is easy to prove existence of examples with just one eigenvalue $\lambda(\omega)$ with $N=1$ for which $(\mathbf{H : 1 2})$ holds, in fact is generic, thanks to the easy form the FGR takes, see formula (1.5) [60] for the NLS.

Remark 1.8. Under (H:1) (H:11) we prove that, in an appropriate coordinate system, some key coefficients of the discrete modes equations are non negative. If (H:12) holds, then these coefficients are positive and our proof tells us that the continuous modes disperse and the discrete ones decay to 0 . We expect (H:12) to hold generically. Our proof extends with minor modifications to the case of small solitons discussed in Remark 1.7, where even the case of just one eigenvalue $\lambda(\omega)$ with $N=1$ (in fact even the case with no eigenvalues) was an open problem.

Remark 1.9. One can envisage extending Theorem 1.3 to moving red and rotating solitons. This would require dropping (H:4) Then, since $3 \omega>m$, one would face the embedded eigenvalue $2 \omega$. Problems arising from the possible failure of the dispersive estimates in Sect. 10.1 might be solvable, considering that 29] proves smoothing estimates in the presence of embedded eigenvalues. However, looking at the nonlinear FGR (which considers multiples of the eigenvalues), we also have the problem that $4 \omega>m+\omega$. So $4 \omega$ belongs to a portion of the spectrum where there is superposition of continuous spectrum of distinct components and the hypothesis $3 \omega>m$ is of no help to avoid this.

Consider $\xi \in \operatorname{ker}\left(\mathcal{H}_{\omega}-\lambda_{j}(\omega)\right)$. One of the requirements for linear stability in Definition 3.4 is that if $\xi \neq 0$ then $\left\langle\xi, \Sigma_{3} \xi^{*}\right\rangle>0$. As it might seem artificial, we prove what follows.

Theorem 1.10. Suppose that $\mathcal{O} \subset(m / 3, m)$. Pick $\omega \in \mathcal{O}$ and let $\phi_{\omega}(x)$ be a standing wave of (1.1). Replace (H:5) with the following assumption: 
(H:5') We assume that $\mathcal{H}_{\omega}$ satisfies all the conditions of Definition 3.4 except for condition (4) which we restate as follows. That is, we assume that for any eigenvalue $\lambda>0$ the quadratic form $\xi \rightarrow\left\langle\xi, \Sigma_{3} \xi^{*}\right\rangle$ is non degenerate in $\operatorname{ker}\left(\mathcal{H}_{\omega}-\lambda\right)$. We assume that there exists at least one eigenvalue $\lambda>0$ such that the quadratic form is non positive in $\operatorname{ker}\left(\mathcal{H}_{\omega}-\lambda\right)$.

Assume $\left(\mathbf{H : 1 )}\left(\mathbf{H : 4 )},(\mathbf{H : 5})\right.\right.$ and $\left(\mathbf{H : 6 )}(\mathbf{H : 1 2})\right.$, Then $\phi_{\omega}(x)$ is orbitally unstable.

We will follow the argument developed in 26] for the NLS. The NLDE is harder than the NLS. For example, the regularity of $\phi_{\omega}$ in $\omega$ for NLDE is unknown. The classical methods to prove orbital stability in [15, 62, 35, 36], based as they are on the positivity of certain functionals, do not apply to NLDE because of the strong indefiniteness of the energy. We already mentioned some initial results for the Dirac equation in $9,10,46$. Like in these articles, we exploit the dispersive properties of the linearizations, adapting the methods used to prove asymptotic stability for the NLS initiated in [56, 157, 12, 13] and developed by a substantial number of authors, see the references in [26]. One of the difficult issues for the NLS, was, and still is, to prove that the energy of the discrete modes associated to the eigenvalues in (H:6) leaks either in the radiation part or in the standing wave. The solution to this problem was initiated in [13, where the eigenvalues are close to the continuous spectrum, and solved in quite general form in [26], see also [2, 25. We recall that there is leaking because, in appropriate coordinates, the nonlinear interaction between discrete and continuous modes yields some dissipative coefficients in the equations of the discrete modes, in a way similar to the classical Fermi Golden Rule (FGR). This phenomenon was first established in special cases for the NLS in [13]. The coefficients were identified generally in [28, which built on 33 . Their dissipative nature was established in 26. We refer to 26 for a discussion of the fact that it is essential to exploit the hamiltonian structure of the equation. For work 27] extending the result in $[26$, to moving ground states see Remark 1.1 .

In this article we follow the same framework of [26] obtaining similar results. In particular the key coefficients in the discrete modes equations are shown to be quadratic forms, see Lemma 11.2, By the energy indefiniteness, see Remark 1.5. the sign of these quadratic forms is unclear. We can overcome this uncertainty if we assume $3 \omega>m$, since in this case there is no superposition of continuous spectrum of distinct components and the quadratic forms are easily proved to be non negative.

We need to develop some of the linear theory of dispersion, which in the case of the NLS had been developed in the course of a decade, see [22, 29. Key to dispersion theory is the proof of smoothing estimates for Schrödinger operators with magnetic potentials in 30. There are two points in the article where the strong indefiniteness of the energy interferes with our method and they are discussed in Remark 1.5. We expects these difficulties to be technical and solvable. Notice that in in [9, 10, 46, these difficulties do not arise because smallness of solitons yields absence of resonances for free and the FGR is not addressed because of their restrictive hypotheses.

The instability result in Theorem 1.10 arises from our desire to justify Assumption (H:5) in our definition of linear stability, see Definition 3.4. The proof of Theorem 1.10 is similar to 24. That is, we show that orbital stability implies asymptotic stability, and we then show that this is incompatible with (H:5'), All the proofs are conditional on (H:12), that is that a certain non negative quantity is actually positive. Presumably this is true generically.

1.5. Notation and preliminaries. We consider spaces

$$
H^{k, s}\left(\mathbb{R}^{3}, \mathbb{C}^{4}\right)=\left\{f \in \mathcal{S}^{\prime}\left(\mathbb{R}^{3}\right),\left\|\langle x\rangle^{s}\langle\nabla\rangle^{k} f\right\|_{2}<\infty\right\}
$$

for $s, k \in \mathbb{R}$ with norm $\|f\|_{H^{k, s}}=\left\|\langle x\rangle^{s}\langle\nabla\rangle^{k} f\right\|_{2}$. Sometimes we will write $H_{x}^{k, s}$ to emphasize the independent variable $x$. If $k=0$, we write $L^{2, s}$ instead of $H^{0, s}$.

For $k \in \mathbb{R}$ and $1 \leq p, q \leq \infty$, the Besov space $B_{p, q}^{k}\left(\mathbb{R}^{3}, \mathbb{C}^{d}\right)$ is the space of all tempered distributions $f \in \mathcal{S}^{\prime}\left(\mathbb{R}^{3}, \mathbb{C}^{d}\right)$ such that

$$
\|f\|_{B_{p, q}^{k}}=\left(\sum_{j \in \mathbb{N}} 2^{j k q}\left\|\varphi_{j} * f\right\|_{p}^{q}\right)^{\frac{1}{q}}<+\infty
$$

with $\widehat{\varphi} \in \mathcal{C}_{0}^{\infty}\left(\mathbb{R}^{n} \backslash\{0\}\right)$ such that $\sum_{j \in \mathbb{Z}} \widehat{\varphi}\left(2^{-j} \xi\right)=1$ for all $\xi \in \mathbb{R}^{3} \backslash\{0\}, \widehat{\varphi}_{j}(\xi)=\widehat{\varphi}\left(2^{-j} \xi\right)$ for all $j \in \mathbb{N}^{*}$ and for all $\xi \in \mathbb{R}^{3}$, and $\widehat{\varphi_{0}}=1-\sum_{j \in \mathbb{N}^{*}} \widehat{\varphi}_{j}$. It is endowed with the norm $\|f\|_{B_{p, q}^{k}}$.

For $A$ a closed operator on a Hilbert space $X$ we will set $R_{A}(z):=(A-z)^{-1}$ for any $z$ in the resolvent set of $A$. 
1.6. Structure of the article. The paper is organized as follows. In Sections 2 4 , we study of the linearization of (1.1) at the stationary solution, we give some information on the spectrum and on symmetries of the linearization, we define the notion of linear stability and we introduce an appropriate coordinate system related to the spectral decomposition of the linearized operator. In Sect. 5 and in the Appendix we discuss estimates on such operators. In Sect. 6 we discuss we reframe the system in a hamiltonian form. In Sect. 7 we look for canonical coordinates. In Sect. 9.2 we reformulate the system in these coordinates. In Sect. 9 we apply the method of Birkhoff normal forms. The proofs of the analogous parts in [26] work almost unaltered. Having chosen an appropriate coordinate system, in Sect 10.1] we begin to prove nonlinear dispersion, in particular estimating the continuous modes. We finish with the closing up of the estimates in Sect. 11 where we prove the Fermi Golden Rule. Specifically we prove that appropriate coefficients are quadratic forms and that for $\omega>m / 3$ they are non negative. Finally, under hypothesis (H:12) which presumably holds generically, we close up the inequalities and we conclude the proof of asymptotic stability, Theorem 1.3. We also prove Theorems 1.10 using similar ideas. In the Appendix we proves smoothing estimates and scattering estimates.

\section{SET UP AND SYMMETRIES}

2.1. Set up. Since our ambient space is $H^{k_{0}}\left(\mathbb{R}^{3}, \mathbb{C}^{4}\right)$ with $k_{0} \geq 4$ and so in particular $k_{0}>3 / 2$, under (H:1) the functional $u \rightarrow g(u \bar{u}) \beta u$ is locally Lipschitz and (1.1) is locally well posed, see pp. 293-294 volume III [58. Consider the solution $u(t, x)$ of (1.1). Then by (H:4) we have $u(t,-x)=\beta u(t, x)$ and $u\left(t,-x_{1},-x_{2}, x_{3}\right)=S_{3} u(t, x)$. We write the ansatz

$$
u(t, x)=e^{\mathrm{i} \vartheta(t)}\left(\phi_{\omega(t)}(x)+r(t, x)\right) .
$$

Inserting (2.1) in (1.1) we get from the definition of $\phi_{\omega}$

$$
\begin{aligned}
& \mathrm{i} r_{t}=D_{m} r-\omega(t) r-g\left(\phi_{\omega(t)} \bar{\phi}_{\omega(t)}\right) \beta r-g^{\prime}\left(\phi_{\omega(t)} \bar{\phi}_{\omega(t)}\right)\left(r \bar{\phi}_{\omega(t)}\right) \beta \phi_{\omega(t)} \\
& -g^{\prime}\left(\phi_{\omega(t)} \bar{\phi}_{\omega(t)}\right)\left(\phi_{\omega(t)} \bar{r}\right) \beta \phi_{\omega(t)}+(\dot{\vartheta}(t)+\omega(t))\left(\phi_{\omega(t)}+r\right)-\mathrm{i} \dot{\omega}(t) \partial_{\omega} \phi_{\omega(t)}+n(r),
\end{aligned}
$$

where $n(r)=O\left(r^{2}\right)$ is defined by

$$
\begin{aligned}
n(r): & =g\left(\left(\phi_{\omega(t)}+r\right) \overline{\phi_{\omega(t)}+r}\right) \beta\left(\phi_{\omega(t)}+r\right)-g\left(\phi_{\omega(t)} \overline{\phi_{\omega(t)}}\right) \beta \phi_{\omega(t)} \\
& -g^{\prime}\left(\phi_{\omega(t)} \bar{\phi}_{\omega(t)}\right)\left(r \bar{\phi}_{\omega(t)}\right) \beta \phi_{\omega(t)}-g^{\prime}\left(\phi_{\omega(t)} \bar{\phi}_{\omega(t)}\right)\left(\phi_{\omega(t)} \bar{r}\right) \beta \phi_{\omega(t)} .
\end{aligned}
$$

We denote by $C: \mathbb{C}^{4} \rightarrow \mathbb{C}^{4}$ the charge conjugation operator $u^{c}:=C u:=\mathrm{i} \beta \alpha_{2} u^{*}$. We have $\alpha_{j} C=C \alpha_{j}$ and $\beta C=-C \beta$ for all $j \in\{1,2,3\}$, [59, Sect. 1.4.6]. Since it is anti-linear, for any $u \in \mathbb{C}^{4}, C\left(u^{*}\right)=(C u)^{*}$.

We state without proof the following simple lemma.

Lemma 2.1. For any vector $v \in \mathbb{C}^{4}$ we have $C^{2} v=v$. Moreover we have:

$$
C(\mathrm{i} v)=-\mathrm{i} v^{c}, \quad v \bar{v}=-C v \overline{C v}, \quad, \quad C(\beta v)=-\beta v^{c}, \quad C\left(D_{m} w\right)=-D_{m} w^{c} .
$$

For $u_{0}$ satisfying $\left(\mathbf{H : 4 )}\right.$ we have $u_{0}^{c}(-x)=-\beta u_{0}^{c}(x)$ and $u_{0}^{c}\left(-x_{1},-x_{2}, x_{3}\right)=-S_{3} u_{0}^{c}(x)$.

Applying $-C$ to (2.2), we obtain

$$
\begin{aligned}
& \mathrm{i} r_{t}^{c}=D_{m} r^{c}+\omega(t) r^{c}-g\left(\phi_{\omega(t)} \bar{\phi}_{\omega(t)}\right) \beta r^{c}+g^{\prime}\left(\phi_{\omega(t)} \bar{\phi}_{\omega(t)}\right)\left(r^{c} \bar{\phi}_{\omega(t)}^{c}\right) \beta \phi_{\omega(t)}^{c} \\
& \quad+g^{\prime}\left(\phi_{\omega(t)} \bar{\phi}_{\omega(t)}\right)\left(\phi_{\omega(t)}^{c} \overline{r^{c}}\right) \beta \phi_{\omega(t)}^{c}-(\dot{\vartheta}(t)+\omega(t))\left(\phi_{\omega(t)}^{c}+r^{c}\right)-\mathrm{i} \dot{\omega}(t) \partial_{\omega} \phi_{\omega(t)}^{c}-C n(r) .
\end{aligned}
$$

We set

$$
\begin{aligned}
& U=\left(\begin{array}{c}
u \\
u^{c}
\end{array}\right) \quad, \quad R=\left(\begin{array}{c}
r \\
r^{c}
\end{array}\right) \quad, \quad \Phi_{\omega}=\left(\begin{array}{c}
\phi_{\omega} \\
\phi_{\omega}^{c}
\end{array}\right) \quad, \quad N(R)=\left(\begin{array}{c}
n(r) \\
-C n(r)
\end{array}\right), \\
& \mathcal{H}_{\omega}=\mathcal{H}_{\omega, 0}+V_{\omega} \quad, \quad \mathcal{H}_{\omega, 0}=\left(\begin{array}{cc}
D_{m}-\omega & 0 \\
0 & D_{m}+\omega
\end{array}\right) \text {, } \\
& V_{\omega}=g\left(\phi_{\omega} \bar{\phi}_{\omega}\right) \beta+g^{\prime}\left(\phi_{\omega} \bar{\phi}_{\omega}\right)\left(\begin{array}{llll}
-\left(\beta \phi_{\omega}^{*}\right. & ) \beta \phi_{\omega} & \left(\beta\left(\phi_{\omega}^{c}\right)^{*}\right. & ) \beta \phi_{\omega} \\
-\left(\beta \phi_{\omega}^{*}\right. & ) \beta \phi_{\omega}^{c} & \left(\beta\left(\phi_{\omega}^{c}\right)^{*}\right. & ) \beta \phi_{\omega}^{c}
\end{array}\right)
\end{aligned}
$$

where the first $\beta$ in the last line is meant in the sense of (2.5) below and where $(\phi \quad)$ stands for the map $r \mapsto \phi r$. Then we have:

$$
\begin{gathered}
\mathrm{i} \dot{R}=\mathcal{H}_{\omega} R+(\dot{\vartheta}(t)+\omega(t))\left(\Sigma_{3} \Phi_{\omega}+\Sigma_{3} R\right)-\mathrm{i} \dot{\omega} \partial_{\omega} \Phi_{\omega}+N(R), \\
\text { where } \Sigma_{1}=\left(\begin{array}{cc}
0 & I_{\mathbb{C}^{4}} \\
I_{\mathbb{C}^{4}} & 0
\end{array}\right), \Sigma_{2}=\left(\begin{array}{cc}
0 & \mathrm{i} I_{\mathbb{C}^{4}} \\
-\mathrm{i} I_{\mathbb{C}^{4}} & 0
\end{array}\right), \Sigma_{3}=\left(\begin{array}{cc}
I_{\mathbb{C}^{4}} & 0 \\
0 & -I_{\mathbb{C}^{4}}
\end{array}\right) .
\end{gathered}
$$


Notice that by $\left(\mathbf{H : 4 )}\right.$ and Lemma 2.1 we have for $\Upsilon(x) \in\left\{\Phi_{\omega}(x), R(t, x)\right\}$

$$
\begin{aligned}
& \Upsilon \Upsilon(-x)=\beta \Sigma_{3} \Upsilon(x) \text { where } \beta=\left(\begin{array}{ll}
\beta & 0 \\
0 & \beta
\end{array}\right) \\
& \text { and } \Upsilon\left(-x_{1},-x_{2}, x_{3}\right)=S_{3} \Sigma_{3} \Upsilon(x) \text { where } S_{3}=\left(\begin{array}{cc}
S_{3} & 0 \\
0 & S_{3}
\end{array}\right) .
\end{aligned}
$$

2.2. Symmetries. We consider now the bilinear map

$$
\left\langle\left(\begin{array}{l}
r_{1} \\
r_{2}
\end{array}\right),\left(\begin{array}{c}
s_{1}^{*} \\
s_{2}^{*}
\end{array}\right)\right\rangle=\int_{\mathbb{R}^{3}}\left(r_{1} \cdot s_{1}^{*}+r_{2} \cdot s_{2}^{*}\right) d x
$$

By $\mathcal{H}_{\omega}^{*}$ we denote the adjoint of $\mathcal{H}_{\omega}$ with respect to this inner product. We have:

Lemma 2.2. We have

$$
\begin{gathered}
\mathcal{H}_{\omega}^{*}=\Sigma_{3} \mathcal{H}_{\omega} \Sigma_{3} \\
\mathcal{H}_{\omega}=-C \Sigma_{1} \mathcal{H}_{\omega} C \Sigma_{1} \text { where } C=\left(\begin{array}{cc}
C & 0 \\
0 & C
\end{array}\right) \\
V_{\omega}(-x)=\beta \Sigma_{3} V_{\omega}(x) \beta \Sigma_{3} \text { with } \beta \text { in the sense of (2.5) } \\
V_{\omega}\left(-x_{1},-x_{2}, x_{3}\right)=S_{3} \Sigma_{3} V_{\omega}(x) S_{3} \Sigma_{3} \text { with } S_{3} \text { in the sense of (2.6) . }
\end{gathered}
$$

Proof. First of all, (2.8)-2.9) hold with $\mathcal{H}_{\omega}$ replaced by $\mathcal{H}_{\omega, 0}$. It remains to check them with $\mathcal{H}_{\omega}$ replaced by $V_{\omega}$. We have $V_{\omega}^{*}=\Sigma_{3} V_{\omega} \Sigma_{3}$ by

$$
\Sigma_{3}\left(\begin{array}{llll}
-\left(\beta \phi_{\omega}^{*}\right. & ) \beta \phi_{\omega} & \left(\beta\left(\phi_{\omega}^{c}\right)^{*}\right. & ) \beta \phi_{\omega} \\
-\left(\beta \phi_{\omega}^{*}\right. & ) \beta \phi_{\omega}^{c} & \left(\beta\left(\phi_{\omega}^{c}\right)^{*}\right. & ) \beta \phi_{\omega}^{c}
\end{array}\right) \Sigma_{3}=\left(\begin{array}{cccc}
-\left(\beta \phi_{\omega}^{*}\right. & ) \beta \phi_{\omega} & -\left(\beta\left(\phi_{\omega}^{c}\right)^{*}\right. & ) \beta \phi_{\omega} \\
\left(\beta \phi_{\omega}^{*}\right. & ) \beta \phi_{\omega}^{c} & \left(\beta\left(\phi_{\omega}^{c}\right)^{*}\right. & ) \beta \phi_{\omega}^{c}
\end{array}\right)
$$

and from the fact that the matrix in rhs(2.12) is the adjoint of the matrix in lhs(2.12). (2.9) holds with $\mathcal{H}_{\omega}$ replaced by $\mathcal{H}_{\omega, 0}$ by Lemma 2.1. We have

$$
\begin{aligned}
& C \Sigma_{1}\left(\begin{array}{llll}
-\left(\beta \phi_{\omega}^{*}\right. & ) \beta \phi_{\omega} & \left(\beta\left(\phi_{\omega}^{c}\right)^{*}\right. & ) \beta \phi_{\omega} \\
-\left(\beta \phi_{\omega}^{*}\right. & ) \beta \phi_{\omega}^{c} & \left(\beta\left(\phi_{\omega}^{c}\right)^{*}\right. & ) \beta \phi_{\omega}^{c}
\end{array}\right)= \\
& -C\left(\begin{array}{llll}
-\left(\beta\left(\phi_{\omega}^{c}\right)^{*}\right. & ) \beta \phi_{\omega}^{c} & \left(\beta \phi_{\omega}^{*}\right. & ) \beta \phi_{\omega}^{c} \\
-\left(\beta\left(\phi_{\omega}^{c}\right)^{*}\right. & ) \beta \phi_{\omega} & \left(\beta \phi_{\omega}^{*}\right. & ) \beta \phi_{\omega}
\end{array}\right) \Sigma_{1}= \\
& -\left(\begin{array}{llll}
\left(\beta\left(\phi_{\omega}^{c}\right)^{*}\right. & )^{*} \beta \phi_{\omega} & -\left(\beta \phi_{\omega}^{*}\right. & )^{*} \beta \phi_{\omega} \\
\left(\beta\left(\phi_{\omega}^{c}\right)^{*}\right. & )^{*} \beta \phi_{\omega}^{c} & -\left(\beta \phi_{\omega}^{*}\right. & )^{*} \beta \phi_{\omega}^{c}
\end{array}\right) \Sigma_{1} .
\end{aligned}
$$

We have for $v \in \mathbb{C}^{4}$

$$
\begin{aligned}
& \left(\beta\left(\phi_{\omega}^{c}\right)^{*} v\right)^{*}=\beta\left(\mathrm{i} \beta \alpha_{2} \phi_{\omega}^{*}\right) v^{*}=-\beta \phi_{\omega}^{*} C(v), \\
& \left(\beta \phi_{\omega}^{*} v\right)^{*}=\beta \phi_{\omega} v^{*}=-\beta\left(\mathrm{i} \beta \alpha_{2} \phi_{\omega}\right)\left(\mathrm{i} \beta \alpha_{2} v^{*}\right)=-\beta\left(\phi_{\omega}^{c}\right)^{*} C(v) .
\end{aligned}
$$

Then

$$
\operatorname{rhs}(2.13)=-\left(\begin{array}{cccc}
-\left(\beta \phi_{\omega}^{*}\right. & ) \beta \phi_{\omega} & \left(\beta\left(\phi_{\omega}^{c}\right)^{*}\right. & ) \beta \phi_{\omega} \\
-\left(\beta \phi_{\omega}^{*}\right. & ) \beta \phi_{\omega}^{c} & \left(\beta\left(\phi_{\omega}^{c}\right)^{*}\right. & ) \beta \phi_{\omega}^{c}
\end{array}\right) C \Sigma_{1} .
$$

This yields (2.9). The proof of (2.10) goes as follows. Using $\phi(-x)=\beta \phi(x)$ and $\phi^{c}(-x)=-\beta \phi^{c}(x)$, where we omit the subindex $\omega$, we have

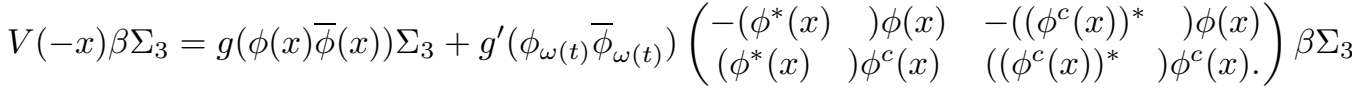

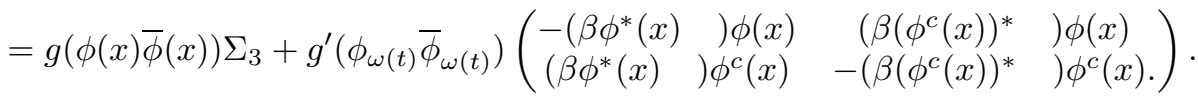

Similarly

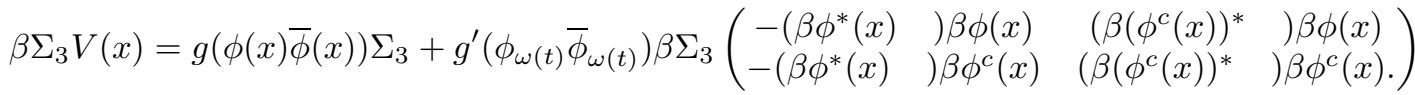

$$
\begin{aligned}
& =\text { second line of (2.14). }
\end{aligned}
$$

The last two formulas yield (2.10). Identity (2.11) is proved similarly. 
Lemma 2.3. For $\mathbf{A}$ the operator in $L^{2}\left(\mathbb{R}^{3}, \mathbb{C}^{8}\right)$ defined by $(\mathbf{A} X)(x):=\beta \Sigma_{3} X(-x)$, then $\mathbf{A}^{2}=I d$, $\mathbf{A}$ is selfadjoint and $\sigma(\mathbf{A})=\{1,-1\}$. We have $\left[\mathbf{A}, \mathcal{H}_{\omega}\right]=\left[\mathbf{A}, \mathcal{H}_{\omega 0}\right]=0$.

For $\mathbf{B}$ the operator in $L^{2}\left(\mathbb{R}^{3}, \mathbb{C}^{8}\right)$ defined by $(\mathbf{B} X)(x):=S_{3} \Sigma_{3} X\left(-x_{1},-x_{2}, x_{3}\right)$, then $\mathbf{B}^{2}=I d, \mathbf{B}$ is selfadjoint and $\sigma(\mathbf{B})=\{1,-1\}$. We have $\left[\mathbf{B}, \mathcal{H}_{\omega}\right]=\left[\mathbf{B}, \mathcal{H}_{\omega 0}\right]=0$.

Moreover, $[\boldsymbol{A}, \boldsymbol{B}]=0$.

Proof. The first sentence is elementary. The second follows by $[\mathbf{A}, A]=0$ for $A=D_{m}, \Sigma_{3}$ (straightforward) and $A=V_{\omega}$, from (2.10). The statements for $\mathbf{B}$ are obtained similarly. $[\mathbf{A}, \mathbf{B}]=0$ is elementary.

2.3. Energy and charge. We have the following elementary result.

Lemma 2.4. Let $U^{T}=(u, C u)$. Set for $G(0)=0$ and $G^{\prime}(s)=g(s)$

$$
\begin{aligned}
& E(U)=E_{K}(U)+E_{P}(U), E_{K}(U)=\int_{\mathbb{R}^{3}}\left(D_{m} u\right) u^{*} d x, E_{P}(U)=-\int_{\mathbb{R}^{3}} G(u \bar{u}) d x, \\
& Q(U)=\int_{\mathbb{R}^{3}} u u^{*} d x
\end{aligned}
$$

Then $E(U)$ and $Q(U)$ are invariants of motion for (1.1) and we have

$$
\begin{aligned}
& E(U)=\frac{1}{2}\left\langle\mathrm{i} \beta \alpha_{2} \Sigma_{3} \Sigma_{1} D_{m} U, U\right\rangle-\int_{\mathbb{R}^{3}} G\left(\frac{1}{2} U \cdot \mathrm{i} \alpha_{2} \Sigma_{3} \Sigma_{1} U\right) d x \\
& Q(U)=\frac{1}{2}\left\langle U, \mathrm{i} \beta \alpha_{2} \Sigma_{1} U\right\rangle,
\end{aligned}
$$

where for $\langle\cdot, \cdot\rangle$ see (2.7). U satisfies system

$$
\mathrm{i} \dot{U}=\mathrm{i} \beta \alpha_{2} \Sigma_{3} \Sigma_{1} \nabla E(U) .
$$

Proof. For any symmetric operator $A$ acting on $L^{2}\left(\mathbb{R}^{3}, \mathbb{C}^{4}\right)$ with the domain invariant by $C$ and anticommuting to $C$ and any $u \in D(A)$,

$$
\begin{aligned}
u \cdot(A u)^{*} & =\frac{u \cdot(A u)^{*}+u^{*} \cdot(A u)}{2}=\frac{u \cdot \mathrm{i} \beta \alpha_{2} C A u+\mathrm{i} \beta \alpha_{2} C u \cdot A u}{2} \\
& =\frac{-u \cdot \mathrm{i} \beta \alpha_{2} A u^{c}+\mathrm{i} \beta \alpha_{2} u^{c} \cdot A u}{2}=\frac{-u \cdot \mathrm{i} \beta \alpha_{2} A u^{c}+u^{c} \mathrm{i} \beta \alpha_{2} \cdot A u}{2} \\
& =\frac{\mathrm{i}}{2} U \cdot \beta \alpha_{2} \Sigma_{3} \Sigma_{1} A U, \text { where we write } A \text { for } A=\left(\begin{array}{cc}
A & 0 \\
0 & A
\end{array}\right) .
\end{aligned}
$$

If $A$ commutes with $C$, then a similar calculation shows $\left\langle u,(A u)^{*}\right\rangle=\frac{\mathrm{i}}{2}\left\langle U, \beta \alpha_{2} \Sigma_{1} A U\right\rangle$. These identities for $A=D_{m}, A=\beta$ or $A=I$ prove the lemma.

\section{Spectrum and Linear stability}

From now on we restrict attention to $\mathbf{X}=\left\{\Upsilon \in L^{2}\left(\mathbb{R}^{3}, \mathbb{C}^{8}\right): \Upsilon(-x) \equiv \beta \Sigma_{3} \Upsilon(x), \Upsilon\left(-x_{1},-x_{2}, x_{3}\right) \equiv\right.$ $\left.S_{3} \Sigma_{3} \Upsilon(x)\right\}:=\operatorname{ker}(\mathbf{A}-I d) \cap \operatorname{ker}(\mathbf{B}-I d) \subset L^{2}\left(\mathbb{R}^{3}, \mathbb{C}^{8}\right)$. It is invariant by $\mathcal{H}_{\omega, 0}$ and $\mathcal{H}_{\omega}$, see Lemma 2.3 We consider the spectrum

$$
\sigma\left(\mathcal{H}_{\omega}\right)=\left\{\lambda \in \mathbb{C}, \mathcal{H}_{\omega}-\lambda I d: \mathbf{X} \cap H^{1}\left(\mathbb{R}^{3}, \mathbb{C}^{8}\right) \mapsto \mathbf{X} \text { is not invertible }\right\}
$$

We summarize what we know about the spectrum.

Lemma 3.1. (1) For the essential spectrum we have, $\sigma_{\mathrm{ess}}\left(\mathcal{H}_{\omega}\right)=(-\infty, \omega-m] \cup[m-\omega,+\infty)$.

(2) For each $z \in \sigma_{p}\left(\mathcal{H}_{\omega}\right)$ the corresponding generalized eigenspace $N_{g}\left(\mathcal{H}_{\omega}-z\right)$ has finite dimension.

(3) If $z \in \sigma\left(\mathcal{H}_{\omega}\right)$ then also $-z \in \sigma\left(\mathcal{H}_{\omega}\right)$.

(4) For the generalized kernel we have $N_{g}\left(\mathcal{H}_{\omega}^{*}\right) \supseteq\left\{\Phi_{\omega}, \Sigma_{3} \partial_{\omega} \Phi_{\omega}\right\}$.

(5) $\partial_{\omega}\left\|\phi_{\omega}\right\|_{2}^{2} \neq 0$ implies that there are no $v$ such that $\mathcal{H}_{\omega} v=\partial_{\omega} \Phi_{\omega}$.

(6) We have $\mathcal{H}_{\omega} Y=-2 \omega Y$ and $\mathcal{H}_{\omega} C \Sigma_{1} Y=2 \omega C \Sigma_{1} Y$ for $Y:=\left(\begin{array}{c}\alpha_{1} \alpha_{2} \alpha_{3} \beta \phi_{\omega} \\ 0\end{array}\right)$. We have $\Upsilon(-x) \equiv$ $-\beta \Sigma_{3} \Upsilon(x)$ for $\Upsilon=Y, C \Sigma_{1} Y$.

Proof. We have that (1) and (2) are consequences of the above discussion. If $z \in \sigma_{\text {ess }}\left(\mathcal{H}_{\omega}\right)$ then $(3)$ is a consequence of (1). If $z$ is an eigenvalue, then (3) is a consequence of (2.9). (4) is a consequence of 
$N_{g}\left(\mathcal{H}_{\omega}\right) \supseteq\left\{\Sigma_{3} \Phi_{\omega}, \partial_{\omega} \Phi_{\omega}\right\}$ which can be seen as follows. By the gauge invariance of the nonlinearity, $G\left(\left(e^{\mathrm{i} \theta} u\right) \overline{\left(e^{\mathrm{i} \theta} u\right)}\right)=G(u \bar{u})$, where $G$ is a primitive of $g$, we have

$$
\mathcal{H}_{\omega}\left(\begin{array}{c}
\mathrm{i} \phi_{\omega} \\
C \mathrm{i} \phi_{\omega}
\end{array}\right)=0 \text { or } \mathcal{H}_{\omega} \Sigma_{3} \Phi_{\omega}=0
$$

Then differentiating with respect to $\omega$ (1.1) and taking its image by $C$, we obtain $\mathcal{H}_{\omega} \partial_{\omega} \Phi_{\omega}=-\Sigma_{3} \Phi_{\omega}$. (5) follows by the following argument, if we assume existence of $v$ s.t. $\mathcal{H}_{\omega} v=\partial_{\omega} \Phi_{\omega}$,

$$
\begin{aligned}
& 0=\left\langle v,\left(\mathcal{H}_{\omega}^{*} \Phi_{\omega}\right)^{*}\right\rangle=\left\langle\partial_{\omega} \Phi_{\omega}, \Phi_{\omega}^{*}\right\rangle=\left\langle\partial_{\omega} \phi_{\omega}, \phi_{\omega}^{*}\right\rangle+\left\langle\partial_{\omega} \mathrm{i} \beta \alpha_{2} \phi_{\omega}^{*}, \mathrm{i} \beta \alpha_{2} \phi_{\omega}\right\rangle \\
& =\left\langle\partial_{\omega} \phi_{\omega}, \phi_{\omega}^{*}\right\rangle+\left\langle\partial_{\omega} \phi_{\omega}^{*}, \phi_{\omega}\right\rangle=\partial_{\omega}\left\|\phi_{\omega}\right\|_{2}^{2} \neq 0 .
\end{aligned}
$$

(6) is obtained by a direct computation.

Remark 3.2. From (2.8), if $z \in \sigma\left(\mathcal{H}_{\omega}\right)$ then $\bar{z} \in \sigma\left(\mathcal{H}_{\omega}\right)$. So if $z \in \sigma\left(\mathcal{H}_{\omega}\right)$ then $\{z,-z, \bar{z},-\bar{z}\} \subseteq \sigma\left(\mathcal{H}_{\omega}\right)$.

Remark 3.3. The observation that $2 \omega$ is an eigenvalue of $\mathcal{H}_{\omega}$ in $L^{2}\left(\mathbb{R}^{3}, \mathbb{C}^{2}\right)$ is due to 20 . For $3 \omega>m$ the eigenvalue $2 \omega$ is embedded in the continuous spectrum. The fact that the vectors in Claim (6) Lemma 3.1 do not satisfy the symmetry (2.5) and are not in $\mathbf{X}$, shows that the existence of this eigenvalue does not interfere with our proof. Obviously the symmetry (H:4) is crucial.

We have the beginning of $\mathcal{H}_{\omega}$ invariant Jordan block decomposition $\mathbf{X}=N_{g}\left(\mathcal{H}_{\omega}\right) \oplus N_{g}^{\perp}\left(\mathcal{H}_{\omega}^{*}\right)$. Linear stability means to us what follows, see [24].

Definition 3.4 (Linear Stability). A standing wave $e^{\mathrm{it} \omega} \phi_{\omega}$ is linearly stable when the following hold:

(1) $\sigma\left(\mathcal{H}_{\omega}\right) \subset \mathbb{R}$

(2) $N_{g}(\mathcal{H})=\left\{\Sigma_{3} \Phi_{\omega}, \partial_{\omega} \Phi_{\omega}\right\}$

(3) for any eigenvalue $z \neq 0$ of $\mathcal{H}_{\omega}$ we have $N_{g}\left(\mathcal{H}_{\omega}-z\right)=\operatorname{ker}\left(\mathcal{H}_{\omega}-z\right)$;

(4) for any positive eigenvalue $\lambda>0$ and for any $\xi \in \operatorname{ker}\left(\mathcal{H}_{\omega}-\lambda\right)$, we have $\left\langle\xi, \Sigma_{3} \xi^{*}\right\rangle>0$.

As a consequence of (H:5), the Jordan decomposition can be continued as follows:

$$
\begin{aligned}
& \mathbf{X}=N_{g}\left(\mathcal{H}_{\omega}\right) \oplus\left(\oplus_{j, \pm} \operatorname{ker}\left(\mathcal{H}_{\omega} \mp \lambda_{j}(\omega)\right)\right) \oplus L_{c}^{2}\left(\mathcal{H}_{\omega}\right) \text { with } \mathbf{X}_{c}\left(\mathcal{H}_{\omega}\right)=\left\{\mathbf{X}_{d}\left(\mathcal{H}_{\omega}^{*}\right)\right\}^{\perp} \cap \mathbf{X} \\
& \text { where for } K=\mathcal{H}_{\omega}^{*}, \mathcal{H}_{\omega} \text { we set } \mathbf{X}_{d}(K):=N_{g}(K) \oplus \oplus_{j, \pm} \operatorname{ker}\left(K \mp \lambda_{j}(\omega)\right) .
\end{aligned}
$$

Let $\left(\xi_{j}(\omega, x)\right)_{j}$ be a basis of $\oplus_{j=1}^{n} \operatorname{ker}\left(\mathcal{H}_{\omega}-\lambda_{j}(\omega)\right)$ so that each vector is smooth in both variables, with $\left|\partial_{\omega x}^{\alpha} \xi_{j}(\omega, x)\right|<c_{\alpha} e^{-a_{\alpha}|x|}$ for some $c_{\alpha}>0$ and $a_{\alpha}>0$. This can be proved by the Combes-Thomas method [38] using (H:2). We normalize $\xi_{j}(\omega, x)$ so that $\varepsilon_{j}=\left\langle\xi_{j}, \Sigma_{3} \xi_{j}^{*}\right\rangle \in\{1,-1\}$ and $\left\langle\xi_{j}, \Sigma_{3} \xi_{i}^{*}\right\rangle=0$ for $j \neq i$. In Theorem 1.3 for all $\mathrm{j}$ we have $\varepsilon_{j}=1$ while for Theorem 1.10 we have $\varepsilon_{j}=-1$ for at least one $j$.

From the calculations of this section, we have built a dual basis. Hence, given any vector $X$, we have $(3.2)$

$$
\begin{aligned}
& X=\frac{\left\langle X,\left(e^{\mathrm{i} \Sigma_{3} \vartheta} \Sigma_{3} \partial_{\omega} \Phi\right)^{*}\right\rangle}{q^{\prime}(\omega)} e^{\mathrm{i} \Sigma_{3} \vartheta} \Sigma_{3} \Phi+\frac{\left\langle X,\left(e^{\mathrm{i} \Sigma_{3} \vartheta} \Phi\right)^{*}\right\rangle}{q^{\prime}(\omega)} e^{\mathrm{i} \Sigma_{3} \vartheta} \partial_{\omega} \Phi+ \\
& \sum_{j=1}^{n} \varepsilon_{j}\left\langle X,\left(e^{\mathrm{i} \Sigma_{3} \vartheta} \Sigma_{3} \xi_{j}\right)^{*}\right\rangle e^{\mathrm{i} \Sigma_{3} \vartheta} \xi_{j}+\sum_{j=1}^{n} \varepsilon_{j}\left\langle X,\left(e^{\mathrm{i} \Sigma_{3} \vartheta} \Sigma_{1} \Sigma_{3} C \xi_{j}\right)^{*}\right\rangle e^{\mathrm{i} \Sigma_{3} \vartheta} \Sigma_{1} C \xi_{j}+e^{\mathrm{i} \Sigma_{3} \vartheta} P_{c}\left(\mathcal{H}_{\omega}\right) e^{-\mathrm{i} \Sigma_{3} \vartheta} X,
\end{aligned}
$$

with $P_{c}\left(\mathcal{H}_{\omega}\right)$ the projector onto $\mathbf{X}_{c}\left(\mathcal{H}_{\omega}\right)$ with respect to decomposition (3.1). More generally, for $X \in$ $L^{2}\left(\mathbb{R}^{3}, \mathbb{C}^{8}\right)=\mathbf{X} \oplus \mathbf{X}^{\perp}$, see the simultaneous spectral decomposition of $\mathbf{A}$ and $\mathbf{B}$ in Lemma 2.3. we denote by $P_{c}\left(\mathcal{H}_{\omega}\right) X$ the vector obtained first projecting in $\mathbf{X}$ and then in $\mathbf{X}_{c}\left(\mathcal{H}_{\omega}\right)$. By duality, we have the following lemma.

Lemma 3.5. Suppose that for a given $\omega \in \mathcal{O}$ the conditions of Definition 3.4 are satisfied. Then

$$
\mathbf{X}=N_{g}\left(\mathcal{H}_{\omega}^{*}\right) \oplus\left(\oplus_{j, \pm} \operatorname{ker}\left(\mathcal{H}_{\omega}^{*} \mp \lambda_{j}(\omega)\right)\right) \oplus \mathbf{X}_{c}\left(\mathcal{H}_{\omega}^{*}\right) \text { with } \mathbf{X}_{c}\left(\mathcal{H}_{\omega}^{*}\right):=\left\{\mathbf{X}_{d}\left(\mathcal{H}_{\omega}\right)\right\}^{\perp}
$$

Any 1 form $\alpha=\left\langle\alpha^{\sharp}, \quad\right\rangle$ can be decomposed as follows:

$$
\begin{aligned}
& \alpha^{\sharp}=\frac{\left\langle\alpha^{\sharp}, e^{\mathrm{i} \Sigma_{3} \vartheta} \partial_{\omega} \Phi\right\rangle}{q^{\prime}(\omega)}\left(e^{\mathrm{i} \Sigma_{3} \vartheta} \Phi\right)^{*}+\frac{\left\langle\alpha^{\sharp}, e^{\mathrm{i} \Sigma_{3} \vartheta} \Sigma_{3} \Phi\right\rangle}{q^{\prime}(\omega)}\left(e^{\mathrm{i} \Sigma_{3} \vartheta} \Sigma_{3} \partial_{\omega} \Phi\right)^{*} \\
& +\sum_{j=1}^{n} \varepsilon_{j}\left\langle\alpha^{\sharp}, e^{\mathrm{i} \Sigma_{3} \vartheta} \xi_{j}\right\rangle\left(e^{\mathrm{i} \Sigma_{3} \vartheta} \Sigma_{3} \xi_{j}\right)^{*}-\sum_{j=1}^{n} \varepsilon_{j}\left\langle\alpha^{\sharp}, e^{\mathrm{i} \Sigma_{3} \vartheta} \Sigma_{1} C \xi_{j}\right\rangle\left(e^{\mathrm{i} \Sigma_{3} \vartheta} \Sigma_{3} \Sigma_{1} C \xi_{j}\right)^{*} \\
& +e^{-\mathrm{i} \Sigma_{3} \vartheta}\left(P_{c}\left(\mathcal{H}_{\omega}^{*}\right) e^{-\mathrm{i} \Sigma_{3} \vartheta}\left(\alpha^{\sharp}\right)^{*}\right)^{*} .
\end{aligned}
$$




\section{Modulation And COORDinates}

4.1. Modulation. Consider the $U$ in (2.3). Then, in the notation of (2.3), (2.1) can be written as

$$
U=e^{\mathrm{i} \Sigma_{3} \vartheta}\left(\Phi_{\omega}+R\right) .
$$

Consider the following two functions

$$
\mathcal{F}(U, \omega, \vartheta):=\left\langle e^{-\mathrm{i} \Sigma_{3} \vartheta} U-\Phi_{\omega}, \Phi_{\omega}^{*}\right\rangle, \quad \mathcal{G}(U, \omega, \vartheta):=\left\langle e^{-\mathrm{i} \Sigma_{3} \vartheta} U, \Sigma_{3} \partial_{\omega} \Phi_{\omega}^{*}\right\rangle
$$

Notice that $R \in N_{g}^{\perp}\left(\mathcal{H}_{\omega}^{*}\right)$ if and only if $\mathcal{F}(U, \omega, \vartheta)=\mathcal{G}(U, \omega, \vartheta)=0$. By (H:2) the map $\omega \in \mathcal{O} \rightarrow \phi_{\omega} \in$ $H^{1}\left(\mathbb{R}^{3}\right)$ is $C^{\infty}$. Then $\mathcal{F}$ and $\mathcal{G}$ are $C^{\infty}$ functions with partial derivatives

$$
\begin{aligned}
& \mathcal{F}_{\vartheta}(U, \omega, \vartheta)=-\mathrm{i}\left\langle\Sigma_{3} e^{-\mathrm{i} \Sigma_{3} \vartheta} U, \Phi_{\omega}^{*}\right\rangle \\
& \mathcal{F}_{\omega}(U, \omega, \vartheta)=-2 q^{\prime}(\omega)+\left\langle e^{-\mathrm{i} \Sigma_{3} \vartheta} U, \partial_{\omega} \Phi_{\omega}^{*}\right\rangle \\
& \mathcal{F}_{U}(U, \omega, \vartheta)=e^{-\mathrm{i} \Sigma_{3} \vartheta} \Phi_{\omega}^{*}, \quad \mathcal{G}_{U}(U, \omega, \vartheta)=e^{-\mathrm{i} \Sigma_{3} \vartheta} \Sigma_{3} \partial_{\omega} \Phi_{\omega}^{*} \\
& \mathcal{G}_{\vartheta}(U, \omega, \vartheta)=-\mathrm{i}\left\langle e^{-\mathrm{i} \Sigma_{3} \vartheta} U, \partial_{\omega} \Phi_{\omega}^{*}\right\rangle, \quad \mathcal{G}_{\omega}(U, \omega, \vartheta)=\left\langle e^{-\mathrm{i} \Sigma_{3} \vartheta} U, \Sigma_{3} \partial_{\omega}^{2} \Phi_{\omega}^{*}\right\rangle .
\end{aligned}
$$

We have $\mathcal{F}\left(e^{\mathrm{i} \Sigma_{3} \vartheta} \Phi_{\omega}, \omega, \vartheta\right)=\mathcal{G}\left(e^{\mathrm{i} \Sigma_{3} \vartheta} \Phi_{\omega}, \omega, \vartheta\right)=0$. For $U=e^{\mathrm{i} \Sigma_{3} \vartheta} \Phi_{\omega}$ in (4.2) we get

$$
\begin{aligned}
& \mathcal{F}_{\vartheta}\left(e^{\mathrm{i} \Sigma_{3} \vartheta} \Phi_{\omega}, \omega, \vartheta\right)=0 \quad, \quad \mathcal{F}_{\omega}(U, \omega, \vartheta)=-q^{\prime}(\omega), \\
& \mathcal{G}_{\vartheta}\left(e^{\mathrm{i} \Sigma_{3} \vartheta} \Phi_{\omega}, \omega, \vartheta\right)=-\mathrm{i} q^{\prime}(\omega) \quad, \quad \mathcal{G}_{\omega}\left(e^{\mathrm{i} \Sigma_{3} \vartheta} \Phi_{\omega}, \omega, \vartheta\right)=0 .
\end{aligned}
$$

Then by the implicit function theorem and $(\mathbf{H : 3})$ there is a unique choice of functions $\theta=\theta(U), \omega=\omega(U)$ which are $C^{\infty}$ and yield to the following lemma.

Lemma 4.1 (Modulation). For any $\omega_{1} \in \mathcal{O}$ there exist $\varepsilon>0$ and $C>0$ such that for any $u \in H^{1}\left(\mathbb{R}^{3}\right)$ with $\left\|u-e^{\mathrm{i} \vartheta_{1}} \phi_{\omega_{1}}\right\|<\epsilon<\varepsilon$, there exists a unique choice of $(\vartheta, \omega, r)$ such that $\left|\omega-\omega_{1}\right|+\left|\vartheta-\vartheta_{1}\right|<C \epsilon$ for a fixed $C, R \in N_{g}^{\perp}\left(\mathcal{H}_{\omega}^{*}\right)$ and (4.1) hold.

Consider the two $C^{\infty}$ functions $\vartheta, \omega: U \in B_{H^{1}}\left(e^{i \Sigma \vartheta_{0}} \Phi_{\omega_{1}}, \varepsilon\right) \rightarrow \mathbb{R}$. Inserting (4.1) in (4.2) we get

$$
\begin{aligned}
& \mathcal{F}_{\vartheta}=-\mathrm{i}\left\langle\Sigma_{3} R, \Phi_{\omega}^{*}\right\rangle ; \quad \mathcal{F}_{\omega}=-q^{\prime}(\omega)+\left\langle R, \partial_{\omega} \Phi_{\omega}^{*}\right\rangle ; \\
& \mathcal{F}_{U}=e^{-\mathrm{i} \Sigma_{3} \vartheta} \Phi_{\omega}^{*} ; \quad \mathcal{G}_{U}=e^{-\mathrm{i} \Sigma_{3} \vartheta} \Sigma_{3} \partial_{\omega} \Phi_{\omega}^{*} ; \\
& \mathcal{G}_{\vartheta}=-\mathrm{i}\left(q^{\prime}(\omega)+\left\langle R, \partial_{\omega} \Phi_{\omega}^{*}\right\rangle\right) ; \quad \mathcal{G}_{\omega}=\left\langle R, \Sigma_{3} \partial_{\omega}^{2} \Phi_{\omega}^{*}\right\rangle .
\end{aligned}
$$

Then, if we set

$$
\mathcal{A}:=\left(\begin{array}{cc}
-q^{\prime}(\omega)+\left\langle R, \partial_{\omega} \Phi_{\omega}^{*}\right\rangle & -\mathrm{i}\left\langle\Sigma_{3} R, \Phi_{\omega}^{*}\right\rangle \\
\left\langle R, \Sigma_{3} \partial_{\omega}^{2} \Phi_{\omega}^{*}\right\rangle & -\mathrm{i}\left(q^{\prime}(\omega)+\left\langle R, \partial_{\omega} \Phi_{\omega}^{*}\right\rangle\right)
\end{array}\right)
$$

we have the following equality

$$
\mathcal{A}\left(\begin{array}{c}
\nabla \omega \\
\nabla \vartheta
\end{array}\right)=\left(\begin{array}{c}
-e^{-\mathrm{i} \Sigma_{3} \vartheta} \Phi_{\omega}^{*} \\
-e^{-\mathrm{i} \Sigma_{3} \vartheta} \Sigma_{3} \partial_{\omega} \Phi_{\omega}^{*}
\end{array}\right)
$$

where given a vector field $X$ and a scalar valued function $F$, we have $X F=\langle\nabla F, X\rangle=d F(X)$, with $d F$ the exterior differential and $\nabla F$ the gradient.

By the above discussion we obtain the following lemma.

Lemma 4.2. We have the following formulas:

$$
\begin{aligned}
& \nabla \omega=\frac{\left(q^{\prime}(\omega)+\left\langle R, \partial_{\omega} \Phi_{\omega}^{*}\right\rangle\right)\left(e^{\mathrm{i} \Sigma_{3} \vartheta} \Phi\right)^{*}-\left\langle\Sigma_{3} R, \Phi_{\omega}^{*}\right\rangle\left(e^{\mathrm{i} \Sigma_{3} \vartheta} \Sigma_{3} \partial_{\omega} \Phi\right)^{*}}{\left(q^{\prime}(\omega)\right)^{2}-\left\langle R, \partial_{\omega} \Phi_{\omega}^{*}\right\rangle^{2}+\left\langle\Sigma_{3} R, \Phi_{\omega}^{*}\right\rangle\left\langle R, \Sigma_{3} \partial_{\omega}^{2} \Phi_{\omega}^{*}\right\rangle} \\
& \nabla \vartheta=\frac{\left\langle R, \Sigma_{3} \partial_{\omega}^{2} \Phi_{\omega}^{*}\right\rangle\left(e^{\mathrm{i} \Sigma_{3} \vartheta} \Phi\right)^{*}+\left(q^{\prime}(\omega)-\left\langle R, \partial_{\omega} \Phi_{\omega}^{*}\right\rangle\right)\left(e^{\mathrm{i} \Sigma_{3} \vartheta} \Sigma_{3} \partial_{\omega} \Phi\right)^{*}}{\left.\mathrm{i}\left[q^{\prime}(\omega)\right)^{2}-\left\langle R, \partial_{\omega} \Phi_{\omega}^{*}\right\rangle^{2}+\left\langle\Sigma_{3} R, \Phi_{\omega}^{*}\right\rangle\left\langle R, \Sigma_{3} \partial_{\omega}^{2} \Phi_{\omega}^{*}\right\rangle\right]} .
\end{aligned}
$$


4.2. Coordinates. For $\omega \in \mathcal{O}$ we consider decomposition (3.1). By $P_{c}\left(\mathcal{H}_{\omega}\right)$ (resp. $P_{d}\left(\mathcal{H}_{\omega}\right)$ ), or simply by $P_{c}(\omega)$ (resp. $\left.P_{d}(\omega)\right)$, we denote the projection on $\mathbf{X}_{c}\left(\mathcal{H}_{\omega}\right)$ (resp. $\left.\mathbf{X}_{d}\left(\mathcal{H}_{\omega}\right)\right)$. The space $\mathbf{X}_{c}\left(\mathcal{H}_{\omega}\right)$ "depends continuously" on $\omega$, as $P_{c}(\omega)=1-P_{d}(\omega)$ depends smoothly on $\omega$.

By Lemma 4.1] we specify the ansatz (4.1) imposing $\omega \in \mathcal{O}, \vartheta \in \mathbb{R}$ and $R \in N_{g}^{\perp}\left(\mathcal{H}_{\omega}^{*}\right)$. Fix $\omega_{0}$, where $q\left(\omega_{0}\right)=\left\|u_{0}\right\|_{L^{2}}^{2}$. For $\omega$ close to $\omega_{0}$ the map $P_{c}\left(\mathcal{H}_{\omega}\right)$ is an isomorphism from $\mathbf{X}_{c}\left(\mathcal{H}_{\omega_{0}}\right)$ to $\mathbf{X}_{c}\left(\mathcal{H}_{\omega}\right)$. In particular we write $\left(\bar{z}_{j}\right.$ is the complex conjugate of the scalar $\left.z_{j}\right)$

$$
N_{g}^{\perp}\left(\mathcal{H}_{\omega}^{*}\right) \text { э } R=\sum_{j=1}^{n} z_{j} \xi_{j}(\omega)+\sum_{j=1}^{n} \bar{z}_{j} \Sigma_{1} C \xi_{j}(\omega)+P_{c}\left(\mathcal{H}_{\omega}\right) f, \quad f \in \mathbf{X}_{c}\left(\mathcal{H}_{\omega_{0}}\right) .
$$

Setting $z \cdot \xi=\sum_{j=1}^{n} z_{j} \xi_{j}$ and $\bar{z} \cdot \Sigma_{1} C \xi=\sum_{j=1}^{n} \bar{z}_{j} \Sigma_{1} C \xi_{j}$, we write

$$
U=e^{\mathrm{i} \Sigma_{3} \vartheta}\left(\Phi_{\omega}+z \cdot \xi(\omega)+\bar{z} \cdot \Sigma_{1} C \xi(\omega)+P_{c}\left(\mathcal{H}_{\omega}\right) f\right)
$$

$\omega \in \mathcal{O}$ close to $\omega_{0},(z, f) \in \mathbb{C}^{n} \times \mathbf{X}_{c}\left(\mathcal{H}_{\omega_{0}}\right)$ close to 0 , are our coordinates. In the sequel, we set

$$
\partial_{\omega} R:=\sum_{j=1}^{n} z_{j} \partial_{\omega} \xi_{j}(\omega)+\sum_{j=1}^{n} \bar{z}_{j} \Sigma_{1} C \partial_{\omega} \xi_{j}(\omega)+\partial_{\omega} P_{c}\left(\mathcal{H}_{\omega}\right) f .
$$

Then we have the vector fields

$$
\begin{aligned}
& \frac{\partial}{\partial \omega}=e^{\mathrm{i} \Sigma_{3} \vartheta} \partial_{\omega}(\Phi+R), \frac{\partial}{\partial \vartheta}=\mathrm{i} e^{\mathrm{i} \Sigma_{3} \vartheta} \Sigma_{3}(\Phi+R), \\
& \frac{\partial}{\partial z_{j}}=e^{\mathrm{i} \Sigma_{3} \vartheta} \xi_{j}, \frac{\partial}{\partial \bar{z}_{j}}=e^{\mathrm{i} \Sigma_{3} \vartheta} \Sigma_{1} C \xi_{j} .
\end{aligned}
$$

In particular, given a scalar function $F$, we have

$$
\begin{aligned}
& \partial_{\omega} F=\left\langle\nabla F, e^{\mathrm{i} \Sigma_{3} \vartheta} \partial_{\omega}(\Phi+R)\right\rangle, \partial_{\vartheta} F=\mathrm{i}\left\langle\nabla F, e^{\mathrm{i} \Sigma_{3} \vartheta} \Sigma_{3}(\Phi+R)\right\rangle, \\
& \partial_{z_{j}} F=\left\langle\nabla F, e^{\mathrm{i} \Sigma_{3} \vartheta} \xi_{j}\right\rangle, \partial_{\bar{z}_{j}} F=\left\langle\nabla F, e^{\mathrm{i} \Sigma_{3} \vartheta} \Sigma_{1} C \xi_{j}\right\rangle .
\end{aligned}
$$

Lemma 4.3. We have the following formulas:

$$
\begin{aligned}
& \varepsilon_{j} \nabla z_{j}=-\left\langle\Sigma_{3} \xi_{j}^{*}, \partial_{\omega} R\right\rangle \nabla \omega-\mathrm{i}\left\langle\Sigma_{3} \xi_{j}^{*}, \Sigma_{3} R\right\rangle \nabla \vartheta+e^{-\mathrm{i} \Sigma_{3} \vartheta} \Sigma_{3} \xi_{j}^{*} \\
& \varepsilon_{j} \nabla \bar{z}_{j}=-\left\langle\Sigma_{1} \Sigma_{3}\left(C \xi_{j}\right)^{*}, \partial_{\omega} R\right\rangle \nabla \omega-\mathrm{i}\left\langle\Sigma_{1} \Sigma_{3}\left(C \xi_{j}\right)^{*}, \Sigma_{3} R\right\rangle \nabla \vartheta+e^{-\mathrm{i} \Sigma_{3} \vartheta} \Sigma_{1} \Sigma_{3}\left(C \xi_{j}\right)^{*} \\
& f^{\prime}(U)=\left(P_{c}(\omega) P_{c}\left(\omega_{0}\right)\right)^{-1} P_{c}(\omega)\left[-\partial_{\omega} R d \omega-\mathrm{i} \Sigma_{3} R d \vartheta+e^{-\mathrm{i} \Sigma_{3} \vartheta} \mathbb{1}\right],
\end{aligned}
$$

with $\left(P_{c}(\omega) P_{c}\left(\omega_{0}\right)\right)^{-1}: \mathbf{X}_{c}\left(\mathcal{H}_{\omega}\right) \rightarrow \mathbf{X}_{c}\left(\mathcal{H}_{\omega_{0}}\right)$ the inverse of $P_{c}(\omega) P_{c}\left(\omega_{0}\right): \mathbf{X}_{c}\left(\mathcal{H}_{\omega_{0}}\right) \rightarrow \mathbf{X}_{c}\left(\mathcal{H}_{\omega}\right)$ and $\varepsilon_{j}=\left\langle\xi_{j}, \Sigma_{3} \xi_{j}\right\rangle$.

Proof. The proof is similar to the proof of [26, Lemmas 4.1-4.2 ]. Let us see for example the proof of the first formula. Equalities $\frac{\partial z_{j}}{\partial z_{\ell}}=\delta_{j \ell}, \frac{\partial z_{j}}{\partial \bar{z}_{\ell}}=\frac{\partial z_{j}}{\partial \omega}=\frac{\partial z_{j}}{\partial \vartheta}=0$ and $\nabla_{f} z_{j}=0$ are equivalent to

$$
\begin{aligned}
& \left\langle\nabla z_{j}, e^{\mathrm{i} \Sigma_{3} \vartheta} \xi_{\ell}\right\rangle=\delta_{j \ell},\left\langle\nabla z_{j}, e^{\mathrm{i} \Sigma_{3} \vartheta} \Sigma_{1} C \xi_{\ell}\right\rangle \equiv 0=\left\langle\nabla z_{j}, e^{\mathrm{i} \Sigma_{3} \vartheta} \Sigma_{3}(\Phi+R)\right\rangle \\
& \left\langle\nabla z_{j}, e^{\mathrm{i} \Sigma_{3} \vartheta} \partial_{\omega}(\Phi+R)\right\rangle=0 \equiv\left\langle\nabla z_{j}, e^{\mathrm{i} \Sigma_{3} \vartheta} P_{c}(\omega) P_{c}\left(\omega_{0}\right) g\right\rangle \forall g \in \mathbf{X}_{c}\left(\mathcal{H}_{\omega_{0}}\right) .
\end{aligned}
$$

Notice that the last identity implies $P_{c}\left(\mathcal{H}_{\omega_{0}}^{*}\right) P_{c}\left(\mathcal{H}_{\omega}^{*}\right) e^{\mathrm{i} \Sigma_{3} \vartheta} \nabla z_{j}=0$ which in turn implies $P_{c}\left(\mathcal{H}_{\omega}^{*}\right) e^{\mathrm{i} \Sigma_{3} \vartheta} \nabla z_{j}=$ 0 . Then, applying (3.2) and using the product row column, we get for some pair of numbers $(a, b)$

$$
\begin{aligned}
& \nabla z_{j}=a e^{-\mathrm{i} \Sigma_{3} \vartheta} \Phi^{*}+b e^{-\mathrm{i} \Sigma_{3} \vartheta} \Sigma_{3} \partial_{\omega} \Phi^{*}+\varepsilon_{j} e^{-\mathrm{i} \Sigma_{3} \vartheta} \Sigma_{3} \xi_{j}^{*} \\
& =(a, b)\left(\begin{array}{c}
e^{-\mathrm{i} \Sigma_{3} \vartheta} \Phi^{*} \\
e^{-\mathrm{i} \Sigma_{3} \vartheta} \Sigma_{3} \partial_{\omega} \Phi^{*}
\end{array}\right)+\varepsilon_{j} e^{-\mathrm{i} \Sigma_{3} \vartheta} \Sigma_{3} \xi_{j}^{*}=-(a, b) \mathcal{A}\left(\begin{array}{c}
\nabla \omega \\
\nabla \vartheta
\end{array}\right)+\varepsilon_{j} e^{-\mathrm{i} \Sigma_{3} \vartheta} \Sigma_{3} \xi_{j}^{*},
\end{aligned}
$$

where in the last line we used (4.4). Equating the two extreme sides and applying to the formula $\left\langle\cdot, \frac{\partial}{\partial \omega}\right\rangle$ and $\left\langle\cdot, \frac{\partial}{\partial \vartheta}\right\rangle$, by $\left\langle\nabla z_{j}, \frac{\partial}{\partial \omega}\right\rangle=\left\langle\nabla z_{j}, \frac{\partial}{\partial \vartheta}\right\rangle=\left\langle\nabla \vartheta, \frac{\partial}{\partial \omega}\right\rangle=\left\langle\nabla \omega, \frac{\partial}{\partial \vartheta}\right\rangle=0$, by $\left\langle\nabla \vartheta, \frac{\partial}{\partial \vartheta}\right\rangle=\left\langle\nabla \omega, \frac{\partial}{\partial \omega}\right\rangle=1$ and by (4.7) and (4.8), we get

This implies

$$
\mathcal{A}^{*}\left(\begin{array}{l}
a \\
b
\end{array}\right)=\varepsilon_{j}\left(\begin{array}{c}
\left\langle\Sigma_{3} \xi_{j}^{*}, \partial_{\omega} R\right\rangle \\
\mathrm{i}\left\langle\Sigma_{3} \xi_{j}^{*}, \Sigma_{3} R\right\rangle
\end{array}\right)
$$

$$
\nabla z_{j}=-\varepsilon_{j}\left(\left\langle\Sigma_{3} \xi_{j}^{*}, \partial_{\omega} R\right\rangle, \mathrm{i}\left\langle\Sigma_{3} \xi_{j}^{*}, \Sigma_{3} R\right\rangle\right)\left(\begin{array}{c}
\nabla \omega \\
\nabla \vartheta
\end{array}\right)+\varepsilon_{j} e^{-\mathrm{i} \Sigma_{3} \vartheta} \Sigma_{3} \xi_{j}^{*} .
$$




\section{Smoothing AND DisPersive ESTIMATES}

We collect the statements on linear theory needed later to prove the nonlinear estimates.

Lemma 5.1. The following facts are true.

(i) For any $\tau \geq 1$ there exists $C$ independent of $\omega$ s.t.

$$
\begin{aligned}
\left\|R_{D_{m}}(z) \psi\right\|_{L^{2,-\tau}} & \leq C\|\psi\|_{L^{2, \tau}} \text { for all } z \notin \mathbb{R} \\
\left\|R_{\mathcal{H}_{\omega, 0}}(z) \psi\right\|_{L^{2,-\tau}} & \leq C\|\psi\|_{L^{2, \tau}} \text { for all } z \notin \mathbb{R} .
\end{aligned}
$$

(ii) For any $\tau>1$ the following limits

$$
R_{D_{m}}^{+}(\lambda)=\lim _{\varepsilon \searrow 0} R_{D_{m}}(\lambda \pm \mathrm{i} \varepsilon) \text { and } R_{\mathcal{H}_{\omega, 0}}^{+}(\lambda)=\lim _{\varepsilon \searrow 0} R_{\mathcal{H}_{\omega, 0}}(\lambda \pm \mathrm{i} \varepsilon)
$$

exist in $B\left(H_{x}^{1, \tau}, L_{x}^{2,-\tau}\right)$ and the convergence is uniform for $\lambda$ in compact sets.

Proof. Estimate (5.1) implies (5.2). Then (i) is the content of [39, Theorem 2.1] while (ii) is contained in [34, Theorem 1.6].

Lemma 5.2. We have $R_{\mathcal{H}_{\omega, 0}}(x, y, \lambda)=R_{\mathcal{H}_{\omega, 0}}(x-y, \lambda)=\left(\begin{array}{cc}R_{D_{m}}(x-y, \lambda+\omega) & 0 \\ 0 & R_{D_{m}}(x-y, \lambda-\omega)\end{array}\right)$ for $\lambda \notin \sigma\left(\mathcal{H}_{\omega, 0}\right)$ with

$$
R_{D_{m}}(x, \Lambda)=\left(\begin{array}{cc}
(\Lambda+m) I_{2} & \mathrm{i} \sqrt{m^{2}-\Lambda^{2}} \sigma \cdot \widehat{x} \\
\mathrm{i} \sqrt{m^{2}-\Lambda^{2}} \sigma \cdot \widehat{x} & (\Lambda-m) I_{2}
\end{array}\right) \frac{e^{-\sqrt{m^{2}-\Lambda^{2}}|x|}}{4 \pi|x|}+\mathrm{i} \frac{\alpha \cdot \widehat{x}}{4 \pi|x|^{2}} e^{-\sqrt{m^{2}-\Lambda^{2}}|x|}
$$

where $\widehat{x}=x /|x|$ and where for $\zeta=e^{\mathrm{i} \vartheta} r$ with $r \geq 0$ and $\vartheta \in(-\pi, \pi)$ we set $\sqrt{\zeta}=e^{\mathrm{i} \vartheta / 2} \sqrt{r}$.

Proof. This is [59, Identity (1.263) section 1.E].

Remark 5.3. $R_{D_{m}}^{+}(x, \Lambda)$ for $\Lambda>m$ (resp. $\left.\Lambda<-m\right)$ is obtained substituting $\sqrt{m^{2}-\Lambda^{2}}$ in (ㄷ.4) with $-\mathrm{i} \sqrt{\Lambda^{2}-m^{2}}=\lim _{\varepsilon \searrow 0} \sqrt{m^{2}-(\Lambda+\mathrm{i} \varepsilon)^{2}}$ (resp. $\left.\mathrm{i} \sqrt{\Lambda^{2}-m^{2}}=\lim _{\varepsilon \searrow 0} \sqrt{m^{2}-(\Lambda+\mathrm{i} \varepsilon)^{2}}\right)$.

Theorem 5.4. For any $\tau>1$ and $k \in \mathbb{R} \exists C$ s.t.

$$
\begin{aligned}
& \left\|e^{-\mathrm{i} t D_{m}} \psi\right\|_{L_{t}^{2}\left(\mathbb{R}, H^{k,-\tau}\right)} \leq C\|\psi\|_{H^{k}}, \\
& \left\|\int_{\mathbb{R}} e^{\mathrm{i} t D_{m}} F(t) d t\right\|_{H^{k}} \leq C\|F\|_{L_{t}^{2}\left(\mathbb{R}, H^{k, \tau}\right)}, \\
& \left\|\int_{t^{\prime}<t} e^{-\mathrm{i}\left(t-t^{\prime}\right) D_{m}} F\left(t^{\prime}\right) d t^{\prime}\right\|_{L_{t}^{2}\left(\mathbb{R}, H^{k,-\tau}\right)} \leq C\|F\|_{L_{t}^{2}\left(\mathbb{R}, H^{k, \tau}\right)} .
\end{aligned}
$$

The same estimates with the same constants hold when we replace $D_{m}$ with $\mathcal{H}_{\omega, 0}$.

Proof. This is [10, Theorem 1.1] in the free case. But can be easily deduced from Lemma 5.1 using tools in [48, Section XIII.7].

The following theorem is a special case of Theorem 1.1 [9].

Theorem 5.5. For any $\tau>5 / 2$ and $k \in \mathbb{R} \exists C$ s.t. $\left\|e^{-\mathrm{i} t D_{m}} \psi\right\|_{H^{k,-\tau}\left(\mathbb{R}^{3}\right)} \leq C\langle t\rangle^{-\frac{3}{2}}\|\psi\|_{H^{k, \tau}}$. The same estimates with the same constants hold when we replace $D_{m}$ with $\mathcal{H}_{\omega, 0}$.

Theorem 5.6. For any $2 \leq p, q \leq \infty, \theta \in[0,1]$, with $\left(1-\frac{2}{q}\right)\left(1 \pm \frac{\theta}{2}\right)=\frac{2}{p}$ and $(p, \theta) \neq(2,0)$, and for any reals $k, k^{\prime}$ with $k^{\prime}-k \geq \alpha(q)$, where $\alpha(q)=\left(1+\frac{\theta}{2}\right)\left(1-\frac{2}{q}\right)$, there exists a positive constant $C$ such that

$$
\begin{aligned}
& \left\|e^{-\mathrm{i} t D_{m}} \psi\right\|_{L_{t}^{p}\left(\mathbb{R}, B_{q, 2}^{k}\left(\mathbb{R}^{3}, \mathbb{C}^{4}\right)\right)} \leq C\|\psi\|_{H^{k^{\prime}}\left(\mathbb{R}^{3}, \mathbb{C}^{4}\right)}, \\
& \left\|\int e^{\mathrm{i} t D_{m}} F(t) d t\right\|_{H^{k}} \leq C\|F\|_{L_{t}^{p^{\prime}}\left(\mathbb{R}, B_{q^{\prime}, 2}^{k^{\prime}}\left(\mathbb{R}^{3}, \mathbb{C}^{4}\right)\right)}, \\
& \left\|\int_{t^{\prime}<t} e^{-\mathrm{i}\left(t-t^{\prime}\right) D_{m}} F\left(t^{\prime}\right) d t^{\prime}\right\|_{L_{t}^{p}\left(\mathbb{R}, B_{q, 2}^{k}\left(\mathbb{R}^{3}, \mathbb{C}^{4}\right)\right)} \leq C\|F\|_{L_{t}^{a^{\prime}}\left(\mathbb{R}, B_{b^{\prime}, 2}^{h}\left(\mathbb{R}^{3}, \mathbb{C}^{4}\right)\right)},
\end{aligned}
$$

for any $(a, b)$ chosen like $(p, q)$, and $h-k \geq \alpha(q)+\alpha(b)$. Exactly the same estimates hold with $D_{m}$ replaced by $\mathcal{H}_{\omega, 0}$. 
Proof. For $D_{m}$ see [10], see also [14 for the Klein-Gordon case. For $\mathcal{H}_{\omega, 0}$ the statement is an immediate consequence of the case $D_{m}$.

Lemma 5.7. Consider pairs $(p, q)$ as in Theorem 5.6 with $p>2, k \in \mathbb{R}$ arbitrary and $k^{\prime}-k \geq \alpha(q)$. Then for any $\tau>1$ there is a constant $C_{0}=C_{0}(\tau, k, p, q)$ such that

$$
\left\|\int_{0}^{t} e^{\mathrm{i} D_{m}\left(t^{\prime}-t\right)} F\left(t^{\prime}\right) d t^{\prime}\right\|_{L_{t}^{p} B_{q, 2}^{k}} \leq C_{0}\|F\|_{L_{t}^{2} H^{k^{\prime}, \tau}} .
$$

The same estimates hold with $D_{m}$ replaced by $\mathcal{H}_{\omega, 0}$

Proof. For $F(t, x) \in C_{0}^{\infty}\left(\mathbb{R} \times \mathbb{R}^{3}\right)$ set

$$
T F(t)=\int_{0}^{+\infty} e^{\mathrm{i}\left(t^{\prime}-t\right) D_{m}} F\left(t^{\prime}\right) d t^{\prime}, \quad f=\int_{0}^{+\infty} e^{\mathrm{i} t^{\prime} D_{m}} F\left(t^{\prime}\right) d t^{\prime} .
$$

Theorem 5.6 implies $\|T F\|_{L_{t}^{p} B_{q, 2}^{k}} \leq\|f\|_{H^{k^{\prime}}}$ for $k^{\prime}-k=\alpha(q)$. By Theorem 5.4 we have $\|f\|_{H^{k^{\prime}}} \leq$ $C\|F\|_{L_{t}^{2} H^{k^{\prime}, \tau}}$. Since $p>2$, by a well known lemma due to Christ and Kiselev [18], see Lemma 3.1 [53], the statement of Lemma 5.7 follows.

Lemma 5.8. Let $\tau_{1}>1, \mathcal{K}$ a compact subset of $\mathcal{O}$ and $I$ a compact subset of $\sigma_{e}\left(\mathcal{H}_{\omega}\right) \backslash\{ \pm(m \pm \omega)\}$.

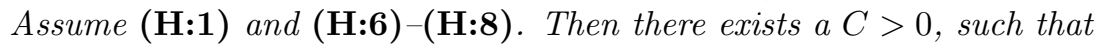

$$
\left\|e^{-\mathrm{i} t \mathcal{H}_{\omega, 0}} R_{\mathcal{H}_{\omega}}^{+}(\lambda) P_{c}(\omega) \psi_{0}\right\|_{L^{2,-\tau_{1}\left(\mathbb{R}^{3}\right)}} \leq C\langle t\rangle^{-\frac{3}{2}}\left\|\psi_{0}\right\|_{L^{2, \tau_{1}+1}\left(\mathbb{R}^{3}\right)}
$$

for every $t \geq 0, \lambda \in I, \omega \in \mathcal{K}$ and $\psi_{0} \in \mathcal{S}\left(\mathbb{R}^{3} ; \mathbb{C}^{2}\right)$.

Proof. We expand $R_{\mathcal{H}_{\omega}}^{+}(\lambda)=R_{\mathcal{H}_{\omega, 0}}^{+}(\lambda)-R_{\mathcal{H}_{\omega 0}}^{+}(\lambda) V_{\omega} R_{\mathcal{H}_{\omega}}^{+}(\lambda)$. We have from [8, Theorem 2 ]

$$
\left\|e^{-\mathrm{i} t \mathcal{H}_{\omega, 0}} R_{\mathcal{H}_{\omega, 0}}^{+}(\lambda) \psi_{0}\right\|_{L^{2,-\tau_{1}\left(\mathbb{R}^{3}\right)}} \leq C\langle t\rangle^{-\frac{3}{2}}\left\|R_{\mathcal{H}_{\omega, 0}}^{+}(\lambda) \psi_{0}\right\|_{L^{2, \tau_{1}\left(\mathbb{R}^{3}\right)}} \leq C_{1}\langle t\rangle^{-\frac{3}{2}}\left\|\psi_{0}\right\|_{L^{2, \tau_{1}+1}\left(\mathbb{R}^{3}\right)},
$$

with $C_{1}$ locally bounded in $\lambda$ and $\tau_{1}$. Hence, by exponential decay of $\phi_{\omega}$ and by (5.5) below,

$$
\begin{aligned}
& \left\|e^{-\mathrm{i} t \mathcal{H}_{\omega, 0}} R_{\mathcal{H}_{\omega, 0}}^{+}(\lambda) V_{\omega} R_{\mathcal{H}_{\omega}}^{+}(\lambda) P_{c}(\omega) \psi_{0}\right\|_{L^{2, \tau_{1}}} \\
& \leq C_{1}\langle t\rangle^{-\frac{3}{2}}\left\|V_{\omega}\right\|_{B\left(L^{\left.2,-\tau_{1}, L^{2, \tau_{1}+1}\right)}\right.}\left\|R_{\mathcal{H}_{\omega}}^{+}(\lambda) P_{c}(\omega)\right\|_{B\left(L^{\left.2, \tau_{1}, L^{2,-\tau_{1}}\right)}\right.}\left\|\psi_{0}\right\|_{L^{2, \tau_{1}}} \leq C^{\prime}\langle t\rangle^{-\frac{3}{2}} .
\end{aligned}
$$

Lemma 5.9. Assume the hypotheses of Lemma 5.8. Then for any $\tau>1$, for any $k \in \mathbb{Z}$ with $k \geq 0$, for a constant $C_{2}=C_{2}(\tau, \omega, k)$ semicontinuous in $\omega$, for any $T>0$ and for any $\forall g(t, x) \in S\left(\mathbb{R}^{4}\right)$, we have

$$
\left\|\int_{0}^{t} e^{-\mathrm{i}(t-s) \mathcal{H}_{\omega}} P_{c}\left(\mathcal{H}_{\omega}\right) g(s, \cdot) d s\right\|_{L_{t}^{2}\left([0, T], H_{x}^{k,-\tau}\right)} \leq C\|g\|_{L_{t}^{2}\left([0, T], H_{x}^{k, \tau}\right)} .
$$

Proof. It is not restrictive to focus only on $T=\infty$ and $k=0$. By Plancherel inequality we have

$$
\begin{aligned}
& \left\|\int_{0}^{t} e^{-\mathrm{i}(t-s) \mathcal{H}_{\omega}} P_{c}\left(\mathcal{H}_{\omega}\right) g(s, \cdot) d s\right\|_{L_{t}^{2} L_{x}^{2,-\tau}} \leq\left\|R_{\mathcal{H}_{\omega}}^{+}(\lambda) P_{c}\left(\mathcal{H}_{\omega}\right) \widehat{\chi}_{[0,+\infty)} *_{\lambda} \widehat{g}(\lambda, x)\right\|_{L_{\lambda}^{2} L_{x}^{2,-\tau}} \\
& \leq\|\| R_{\mathcal{H}_{\omega}}^{+}(\lambda) P_{c}\left(\mathcal{H}_{\omega}\right)\left\|_{B\left(L_{x}^{2, \tau}, L_{x}^{2,-\tau}\right)}\right\| \widehat{\chi}_{[0,+\infty)} *_{\lambda} \widehat{g}(\lambda, x)\left\|_{L_{x}^{2, \tau}}\right\|_{L_{\lambda}^{2}} \\
& \leq\left\|R_{\mathcal{H}_{\omega}}^{+}(\lambda) P_{c}\left(\mathcal{H}_{\omega}\right)\right\|_{L_{\lambda}^{\infty}\left(\mathbb{R}, B\left(L_{x}^{2, \tau}, L_{x}^{2,-\tau}\right)\right)}\|g\|_{L_{t}^{2} L_{x}^{2, \tau}}
\end{aligned}
$$

We are done if we can prove

$$
\left\|R_{\mathcal{H}_{\omega}}^{+}(\lambda) P_{c}\left(\mathcal{H}_{\omega}\right)\right\|_{L_{\lambda}^{\infty}\left(\mathbb{R}, B\left(L_{x}^{2, \tau}, L_{x}^{2,-\tau}\right)\right)} \leq C_{2}
$$

By A.3 and Lemma A.1 we have

$$
\left\|R_{\mathcal{H}_{\omega}}^{+}(\lambda) P_{c}\left(\mathcal{H}_{\omega}\right)\right\|_{B\left(L_{x}^{2, \tau}, L_{x}^{2,-\tau}\right)} \leq\left\|\left(1+A R_{\mathcal{H}_{\omega, 0}}^{+}(\lambda) B^{*}\right)^{-1}\right\|_{B(\mathbf{X}, \mathbf{X})}\left\|R_{\mathcal{H}_{\omega, 0}}^{+}(\lambda)\right\|_{B\left(L_{x}^{2, \tau}, L_{x}^{2,-\tau}\right)} .
$$

To prove (5.6) it is enough to consider $\lambda \in\left(\mathbb{R} \backslash\left[-m+\omega+\delta_{0}, m-\omega-\delta_{0}\right]\right)$ as in (A.4). Then we can exploit inequality (A.4) to bound uniformly in $\lambda$ the first factor in the rhs of (5.6). The proof that $\left\|R_{\mathcal{H}_{\omega, 0}}^{+}(\lambda)\right\|_{B\left(L_{x}^{2, \tau}, L_{x}^{2,-\tau}\right)} \leq C$ for a fixed $C$ is a consequence of $\left\|\lambda R_{-\Delta}^{+}\left(\lambda^{2}\right)\right\|_{B\left(L_{x}^{2, \tau}, L_{x}^{2,-\tau}\right)} \leq C^{\prime}$ and $\left\|\nabla R_{-\Delta}^{+}\left(\lambda^{2}\right)\right\|_{B\left(L_{x}^{2, \tau}, L_{x}^{2,-\tau}\right)} \leq C^{\prime}$ by (A.7) A.8. The last two inequalities are proved in [1] 


\section{Hamiltonian Structure}

The discussion in Sections 6 77 is almost the same of [26, rewritten in the context of the Dirac systems.

6.1. Symplectic structure. We recall that in view of Theorem 1.10 we set $\varepsilon_{j}=\left\langle\xi_{j}, \Sigma_{3} \xi_{j}^{*}\right\rangle$ where $\varepsilon_{j} \in\{1,-1\}$. Notice that in Theorem 1.3 and in [26], we have $\varepsilon_{j} \equiv 1$. Our ambient space is $\mathbf{X}$. We focus only on the subspace formed by the points which satisfy $\Sigma_{1} U=C U$. In view of (2.16), the natural symplectic structure is $\Omega(X, Y):=\left\langle X, \mathrm{i} \beta \alpha_{2} \Sigma_{1} \Sigma_{3} Y\right\rangle$.

The Hamiltonian vector field $X_{G}$ of a scalar function $G$ is defined by the equation $\Omega\left(X_{G}, Y\right)=$ $-\mathrm{i}\langle\nabla G, Y\rangle$ for any vector $Y$ and is $X_{G}=\beta \alpha_{2} \Sigma_{3} \Sigma_{1} \nabla G$.

We call Poisson bracket of a pair of scalar valued functions $F$ and $G$ the scalar valued function

$$
\{F, G\}=\left\langle\nabla F, X_{G}\right\rangle=\mathrm{i} \Omega\left(X_{F}, X_{G}\right)=\mathrm{i} \Omega(\nabla F, \nabla G) .
$$

This can be extended to vector valued function using 1-forms or equivalently defining the extension the following way.

Definition 6.1. Given a function $\mathcal{G}(U)$ with values in $\mathbf{X}_{c}\left(\mathcal{H}_{\omega_{0}}\right)$, a symplectic form $\Omega$ and a scalar function $F(U)$, we define $\{\mathcal{G}, F\}=\mathcal{G}^{\prime}(U) X_{F}(U)$, with $X_{F}$ the Hamiltonian vector field associated to $F$. We set $\{F, \mathcal{G}\}:=-\{\mathcal{G}, F\}$.

Lemma 6.2. Let $Q$ be the function defined by (2.15) and let $X_{Q}$ its Hamiltonian vectorfield of $Q$. Then $X_{Q}=-\frac{\partial}{\partial \vartheta}$. We have the following formulas :

$$
\{Q, \omega\}=0, \quad\{Q, \vartheta\}=1, \quad\left\{Q, z_{j}\right\}=\left\{Q, \bar{z}_{j}\right\}=0, \quad\{Q, f\}=0 .
$$

Proof. (6.2) follows from $X_{Q}=-\frac{\partial}{\partial \vartheta}$. The latter follows from (4.7):

$$
X_{Q}=\beta \alpha_{2} \Sigma_{3} \Sigma_{1} \nabla Q=\beta \alpha_{2} \Sigma_{3} \Sigma_{1} \mathrm{i} \beta \alpha_{2} \Sigma_{1} U=-\mathrm{i} \Sigma_{3} U=-\frac{\partial}{\partial \vartheta} .
$$

6.2. Hamiltonian reformulation of the system. For any scalar function $F$, the time derivative of $F(U(t))$ is $\langle\nabla F(U), \dot{U}\rangle$ and thus if $U$ satisfies (2.16) it is $\{F, E\}$. A similar identity holds for vector valued function and thus as in 26] we write our system as

$$
\dot{\omega}=\{\omega, E\}, \quad \dot{f}=\{f, E\}, \quad \dot{z}_{j}=\left\{z_{j}, E\right\}, \quad \dot{\vartheta}=\{\vartheta, E\} .
$$

For $u_{0}$ the initial datum in (1.1), we introduce a new Hamiltonian for which the stationary solution $\Phi_{\omega_{0}}$, with $q\left(\omega_{0}\right)=\left\|u_{0}\right\|_{L_{x}^{2}}^{2}$, is a critical point :

$$
K(U)=E(U)+\omega(U) Q(U)-\omega(U)\left\|u_{0}\right\|_{L_{x}^{2}}^{2} .
$$

By Lemma 6.2 and since $Q(U)$ is an invariant of the motion, see Lemma 2.4 the solution of the initial value problem in (1.1) solves also

$$
\dot{\omega}=\{\omega, K\}, \quad \dot{f}=\{f, K\}, \quad \dot{z}_{j}=\left\{z_{j}, K\right\}, \quad \dot{\vartheta}-\omega=\{\vartheta, K\} .
$$

By $\frac{\partial}{\partial \vartheta} K=0$ and (6.2) the right hand sides in the equations (6.5) do not depend on $\vartheta$. Hence, if we look at the new system

$$
\dot{\omega}=\{\omega, K\}, \quad \dot{f}=\{f, K\}, \quad \dot{z}_{j}=\left\{z_{j}, K\right\}, \quad \dot{\vartheta}=\{\vartheta, K\},
$$

the evolution of the crucial variables $(\omega, z, f)$ in (6.3) and (6.6) is the same. Therefore, to prove Theorem 1.3 it is sufficient to consider system (6.6).

\section{Application of the Darboux Theorem}

We will show that a resonance phenomenon is responsible for energy leaking from discrete to continuous spectrum. This will be seen in appropriate coordinates system, obtained by means of Birkhoff normal forms. Since the coordinates (4.6) are not canonical for the symplectic form $\Omega$, it is natural to apply Darboux theorem, moving to a different set of coordinates. It is key that our nonlinear Dirac equation remain semilinear. Hence we follow the argument of [26. Section 7], which takes care of this, and to which we refer for more details. 
Strategy of the proof. For $q=q(\omega)=\left\|\phi_{\omega}\right\|_{L^{2}}^{2}$, we introduce the 2-form

$$
\Omega_{0}=\mathrm{i} d \vartheta \wedge d q+\varepsilon_{j} d z_{j} \wedge d \bar{z}_{j}+\left\langle f^{\prime}(U) \cdot, \mathrm{i} \beta \alpha_{2} \Sigma_{3} \Sigma_{1} f^{\prime}(U) \cdot\right\rangle
$$

summing on repeated indexes, with $f(U)$ the function in Lemma $4.3 f^{\prime}(U)$ its Frechét derivative and the last term in (7.1) acting on pairs $(X, Y)$ like $\left\langle f^{\prime}(U) X, \mathrm{i} \beta \alpha_{2} \Sigma_{3} \Sigma_{1} f^{\prime}(U) Y\right\rangle$.

The proof of the Darboux Theorem goes as follows. First consider

$$
\Omega_{\tau}=(1-\tau) \Omega_{0}+\tau \Omega=\Omega_{0}+\tau \widetilde{\Omega} \text { with } \widetilde{\Omega}:=\Omega-\Omega_{0} .
$$

In Lemma 7.1, we check that $\Omega_{0}(U)=\Omega(U)$ at $U=e^{\mathrm{i} \Sigma_{3} \vartheta} \Phi_{\omega_{0}}$. Then $\Omega_{\tau}$ is non degenerate near $e^{\mathrm{i} \Sigma_{3} \vartheta} \Phi_{\omega_{0}}$. One considers a 1 - form $\gamma(\tau, U)$ such that $d \gamma(\tau, U)=\widetilde{\Omega}$ with $\gamma(U)=0$ at $U=e^{\mathrm{i} \Sigma_{3} \vartheta} \Phi_{\omega_{0}}$ (external differentiation will always be on the $U$ variable only) and the vector field $\mathcal{Y}^{\tau}$ such that $i_{\mathcal{Y}^{\tau}} \Omega_{\tau}=-\gamma$. The flow $\mathfrak{F}_{\tau}$ generated by $\mathcal{Y}^{\tau}$, close the points $e^{\mathrm{i} \Sigma_{3} \vartheta} \Phi_{\omega_{0}}$ is defined up to time 1 , and is such that $\mathfrak{F}_{1}^{*} \Omega=\Omega_{0}$ by

$$
\begin{aligned}
& \frac{d}{d \tau}\left(\mathfrak{F}_{\tau}^{*} \Omega_{\tau}\right)=\mathfrak{F}_{\tau}^{*}\left(L_{\mathcal{Y}^{\tau}} \Omega_{\tau}\right)+\mathfrak{F}_{\tau}^{*} \frac{d}{d \tau} \Omega_{\tau}= \\
& =\mathfrak{F}_{\tau}^{*} d\left(i_{\mathcal{Y}^{\tau}} \Omega_{\tau}\right)+\mathfrak{F}_{\tau}^{*} \widetilde{\Omega}=\mathfrak{F}_{\tau}^{*}(-d \gamma+\widetilde{\Omega})=0 .
\end{aligned}
$$

This procedure can be carried out abstractly. But here we need to be careful, choosing $\gamma$ appropriately, because we want the new Hamiltonian $\widetilde{K}=K \circ \mathfrak{F}_{1}$ to be $\vartheta$ invariant and yield a semilinear Dirac equation.

In the sequel of this section all the work is finalized to the correct choice if $\gamma$. In Lemma 7.2 we compute explicitly a differential form $\alpha$ and we make the preliminary choice $\gamma=\alpha$. This is not yet the right choice. By the computations in Lemma 7.3 we find the obstruction to the fact that $\widetilde{K}$ is of the desired type. Lemmas 7.5 7.8 are necessary to find an appropriate solution $F$ of a differential equation in Lemma 7.9. Then $\gamma=\alpha+\mathrm{i} d F$ is the right choice of $\gamma$. In Lemma 7.11 we collect a number of useful estimates for $\mathfrak{F}_{1}$. Lemma 7.12 contains information necessary for the reformulation of our system (8.1)-(8.2).

Preliminary remarks. Note that for $U$ in a sufficiently small neigborhood of $\Phi_{\omega}$, that is $R$ small, from (3.2) the vector fields defined in (4.7) can be completed into a basis of $T_{U} L^{2}$ (tangent space at $U$ ). For any vector $Y \in T_{U} L^{2}$, we have

$$
Y=Y_{\vartheta} \frac{\partial}{\partial \vartheta}+Y_{\omega} \frac{\partial}{\partial \omega}+\sum Y_{j} \frac{\partial}{\partial z_{j}}+\sum Y_{\bar{j}} \frac{\partial}{\partial \bar{z}_{j}}+e^{\mathrm{i} \Sigma_{3} \vartheta} P_{c}(\omega) Y_{f}
$$

and defining the dual basis we set

$$
\begin{gathered}
Y_{\vartheta}=d \vartheta(Y), \quad Y_{\omega}=d \omega(Y), \quad Y_{j}=d z_{j}(Y) \\
Y_{\bar{j}}=d \bar{z}_{j}(Y), \quad Y_{f}=f^{\prime}(U) Y .
\end{gathered}
$$

So similarly, a differential 1-form $\gamma$ decomposes as

$$
\gamma=\gamma^{\vartheta} d \vartheta+\gamma^{\omega} d \omega+\sum \gamma^{j} d z_{j}+\sum \gamma^{\bar{j}} d \bar{z}_{j}+\left\langle\gamma^{f}, f^{\prime} \cdot\right\rangle
$$

where $\left\langle\gamma^{f}, f^{\prime} \cdot\right\rangle$ acts on a vector $Y$ as $\left\langle\gamma^{f}, f^{\prime} Y\right\rangle$, with here $\gamma^{f} \in L_{c}^{2}\left(\mathcal{H}_{\omega_{0}}^{*}\right) ; \gamma^{\vartheta}, \gamma^{\omega}, \gamma^{j}$ and $\gamma^{\bar{j}}$ are in $\mathbb{C}$.

Notice that we are reversing the standard notation on super and subscripts for forms and vector fields.

In the sequel, given a differential 1-form $\gamma$ and a point $U$, we will denote by $\gamma_{U}$ the value of $\gamma$ at $U$.

Given a function $\chi$, denote its hamiltonian vector field with respect to $\Omega_{\tau}$ by $X_{\chi}^{\tau}: i_{X_{\chi}^{\tau}} \Omega_{\tau}=-\mathrm{i} d \chi$. By (7.1) we have $X_{q(\omega)}^{0}=-\frac{\partial}{\partial \vartheta}$.

The proof. We have the following preliminary observation ensuring that $\Omega_{\tau}$ is a non degenerate 2 -form in a neighborhood of $e^{\mathrm{i} \Sigma \Sigma_{3} \vartheta} \Phi_{\omega_{0}}$.

Lemma 7.1. At $U=e^{\mathrm{i} \Sigma_{3} \vartheta} \Phi_{\omega_{0}}$, for any $\vartheta$, we have $\Omega_{0}(U)=\Omega(U)$.

Proof. See also [26, Lemma 7.1]. Using (3.2) we get, summing on repeated indexes,

$$
\begin{aligned}
& \Omega(X, Y)=\left\langle X, \mathrm{i} \beta \alpha_{2} \Sigma_{3} \Sigma_{1} Y\right\rangle= \\
& \frac{1}{q^{\prime}}\left\langle\cdot, e^{-\mathrm{i} \Sigma_{3} \vartheta} \Sigma_{3} \partial_{\omega} \Phi^{*}\right\rangle \wedge\left\langle\cdot, e^{-\mathrm{i} \Sigma_{3} \vartheta} \Phi^{*}\right\rangle(X, Y)+ \\
& +\varepsilon_{j}\left\langle\cdot, e^{-\mathrm{i} \Sigma_{3} \vartheta} \Sigma_{3} \xi_{j}^{*}\right\rangle \wedge\left\langle\cdot, e^{-\mathrm{i} \Sigma_{3} \vartheta} \Sigma_{1} \Sigma_{3}\left(C \xi_{j}\right)^{*}\right\rangle(X, Y) \\
& +\left\langle P_{c}\left(\mathcal{H}_{\omega}\right) e^{-\mathrm{i} \Sigma_{3} \vartheta} X, \mathrm{i} \beta \alpha_{2} \Sigma_{3} \Sigma_{1} P_{c}\left(\mathcal{H}_{\omega}\right) e^{-\mathrm{i} \Sigma_{3} \vartheta} Y\right\rangle .
\end{aligned}
$$

Set

$$
a_{1}:=-\mathrm{i} q^{\prime}+\frac{\operatorname{det} \mathcal{A}}{q^{\prime}}+\left\langle P_{N_{g}^{\perp}\left(\mathcal{H}_{\omega}^{*}\right)} \mathrm{i} \Sigma_{1} R, \mathrm{i} \beta \alpha_{2} \Sigma_{3} \Sigma_{1} \partial_{\omega} R\right\rangle
$$


Then $a_{1}$ is smooth in the arguments $\omega \in \mathcal{O}, z \in \mathbb{C}^{n}$ and $f \in H^{-K^{\prime},-S^{\prime}}$ (see (1.4) for the definition) for any pair $\left(K^{\prime}, S^{\prime}\right)$ with, for $(z, f)$ near 0 ,

$$
\left|a_{1}\right| \leq C\left(K^{\prime}, S^{\prime}\right)\left(|z|+\|f\|_{H^{-K^{\prime},-S^{\prime}}}\right)^{2}
$$

by (4.3). Furthermore $a_{1}$ is imaginary valued. By Lemmas 4.2 and 4.3 , summing on repeated indexes we get

$$
\begin{aligned}
& \Omega=\left(\mathrm{i} q^{\prime}+a_{1}\right) d \vartheta \wedge d \omega+\varepsilon_{j} d z_{j} \wedge d \bar{z}_{j} \\
& +d z_{j} \wedge\left(\left\langle\Sigma_{1} \Sigma_{3}\left(C \xi_{j}\right)^{*}, \partial_{\omega} R\right\rangle d \omega+\mathrm{i}\left\langle\Sigma_{1} \Sigma_{3}\left(C \xi_{j}\right)^{*}, \Sigma_{3} R\right\rangle d \vartheta\right) \\
& -d \bar{z}_{j} \wedge\left(\left\langle\Sigma_{3} \xi_{j}^{*}, \partial_{\omega} R\right\rangle d \omega+\mathrm{i}\left\langle\Sigma_{3} \xi_{j}^{*}, \Sigma_{3} R\right\rangle d \vartheta\right)+ \\
& +\left\langle P_{c}(\omega) P_{c}\left(\omega_{0}\right) f^{\prime} \cdot, \mathrm{i} \beta \alpha_{2} \Sigma_{3} \Sigma_{1} P_{c}(\omega) P_{c}\left(\omega_{0}\right) f^{\prime} \cdot\right\rangle+ \\
& +\left\langle P_{c}(\omega) P_{c}\left(\omega_{0}\right) f^{\prime} \cdot, \mathrm{i} \beta \alpha_{2} \Sigma_{3} \Sigma_{1} P_{c}(\omega) \partial_{\omega} R\right\rangle \wedge d \omega+ \\
& +\mathrm{i}\left\langle P_{c}(\omega) P_{c}\left(\omega_{0}\right) f^{\prime} \cdot, \mathrm{i} \beta \alpha_{2} \Sigma_{3} \Sigma_{1} P_{c}(\omega) \Sigma_{3} R\right\rangle \wedge d \vartheta .
\end{aligned}
$$

At points $U=e^{\mathrm{i} \Sigma_{3} \vartheta} \Phi_{\omega}$, that is for $R=0$, we have

$$
\Omega=\mathrm{i} d \vartheta \wedge d q+\varepsilon_{j} d z_{j} \wedge d \bar{z}_{j}+\left\langle P_{c}(\omega) P_{c}\left(\omega_{0}\right) f^{\prime} \cdot, \mathrm{i} \beta \alpha_{2} \Sigma_{3} \Sigma_{1} P_{c}(\omega) P_{c}\left(\omega_{0}\right) f^{\prime} \cdot\right\rangle .
$$

which at $\omega=\omega_{0}$ gives $\Omega=\Omega_{0}$.

Since $\Omega_{\tau}=\Omega_{0}+\tau\left(\Omega-\Omega_{0}\right)$ with $\tau \in[0,1]$ and $\Omega=\Omega_{0}$ at $e^{\mathrm{i} \Sigma_{3} \vartheta} \Phi_{\omega_{0}}$, and since $\Omega_{0}$ is a non degenerate 2 -form, $\Omega_{\tau}$ is also non degenerate in a neighborhood of $e^{\mathrm{i} \Sigma_{3} \vartheta} \Phi_{\omega_{0}}$. Thus the map $X \mapsto i_{X} \Omega_{\tau}$ from vector fields to 1 -forms is bijective at any point in the neighborhood of $e^{\mathrm{i} \Sigma_{3} \vartheta} \Phi_{\omega_{0}}$. Notice that Lemma 7.1 is claimed at $\omega_{0}$ and not at different standing waves, and that the $e^{\mathrm{i} \Sigma_{3} \vartheta} \Phi_{\omega_{0}}$ are the only stationary solutions preserved by our changes of coordinates.

The next lemma suggests as candidate for the 1 form $\gamma$ the choice $\gamma=\alpha$, for $\alpha$ see below. This is not yet the final choice of $\gamma$.

Lemma 7.2. Consider the forms, summing on repeated indexes,

$$
\begin{aligned}
& \varpi(U) Y:=\frac{1}{2}\left\langle\mathrm{i} \beta \alpha_{2} \Sigma_{3} \Sigma_{1} U, Y\right\rangle \\
& \varpi_{0}(U):=-\mathrm{i} q d \vartheta-\varepsilon_{j} \frac{\bar{z}_{j} d z_{j}-z_{j} d \bar{z}_{j}}{2}+\frac{1}{2}\left\langle f(U), \mathrm{i} \beta \alpha_{2} \Sigma_{3} \Sigma_{1} f^{\prime}(U) \quad\right\rangle .
\end{aligned}
$$

Then $d \varpi_{0}=\Omega_{0}, d \varpi=\Omega$. Set

$$
\alpha(U):=\varpi(U)-\varpi_{0}(U)+d \psi(U) \text { where } \psi(U):=\frac{1}{2}\left\langle\Sigma_{3} \Phi^{*}, R\right\rangle .
$$

We have $\alpha=\alpha^{\vartheta} d \vartheta+\alpha^{\omega} d \omega+\left\langle\alpha^{f}, f^{\prime}\right\rangle$ with

$$
\begin{aligned}
\alpha^{\vartheta}+\frac{\mathrm{i}}{2}\|f\|_{2}^{2}= & -\frac{\mathrm{i}}{2}\left\|z \cdot \xi+\bar{z} \cdot \Sigma_{1} C \xi\right\|_{2}^{2}-\mathrm{i} \Re\left\langle z \cdot \xi+\bar{z} \cdot \Sigma_{1} C \xi,\left(P_{c}(\omega) f\right)^{*}\right\rangle \\
& -\mathrm{i} \Re\left\langle\left(P_{c}(\omega)-P_{c}\left(\omega_{0}\right)\right) f,\left(P_{c}(\omega) f\right)^{*}\right\rangle, \\
\alpha^{\omega}= & -\frac{1}{2}\left\langle R^{*}, \Sigma_{3} \partial_{\omega} R\right\rangle, \\
\alpha^{f}= & \frac{1}{2} \mathrm{i} \beta \alpha_{2} \Sigma_{1} \Sigma_{3} P_{c}\left(\mathcal{H}_{\omega_{0}}\right)\left(P_{c}\left(\mathcal{H}_{\omega}\right)-P_{c}\left(\mathcal{H}_{\omega_{0}}\right)\right) f .
\end{aligned}
$$

Proof. Here the proof is almost the same of [26, Lemma 7.2 ]. We focus on (7.8), the only nontrivial statement. We will sum over repeated indexes. We have

$$
\begin{aligned}
& \varpi=\frac{1}{2}\left\langle e^{-\mathrm{i} \Sigma_{3} \vartheta} \mathrm{i} \beta \alpha_{2} \Sigma_{1} \Sigma_{3} \Phi, \cdot\right\rangle+\frac{1}{2}\left\langle e^{-\mathrm{i} \Sigma_{3} \vartheta} \mathrm{i} \beta \alpha_{2} \Sigma_{1} \Sigma_{3} P_{c}(\omega) f, \cdot\right\rangle \\
& +\frac{1}{2} z_{j}\left\langle e^{-\mathrm{i} \Sigma_{3} \vartheta} \mathrm{i} \beta \alpha_{2} \Sigma_{1} \Sigma_{3} \xi_{j}, \cdot\right\rangle-\frac{1}{2} \bar{z}_{j}\left\langle e^{-\mathrm{i} \Sigma_{3} \vartheta} \mathrm{i} \beta \alpha_{2} \Sigma_{3} C \xi_{j}, \cdot\right\rangle .
\end{aligned}
$$

By Lemma 3.5 and summing on repeated indexes we obtain

$$
\begin{aligned}
& \frac{1}{2}\left\langle e^{-\mathrm{i} \Sigma_{3} \vartheta} \mathrm{i} \beta \alpha_{2} \Sigma_{1} \Sigma_{3} \Phi, \cdot\right\rangle=\frac{\left\langle\frac{1}{2} \mathrm{i} \beta \alpha_{2} \Sigma_{1} \Sigma_{3} \Phi, \partial_{\omega} \Phi\right\rangle}{q^{\prime}(\omega)}\left\langle e^{-\mathrm{i} \Sigma_{3} \vartheta} \Phi^{*}, \cdot\right\rangle \\
& +\frac{\left\langle\frac{1}{2} \mathrm{i} \beta \alpha_{2} \Sigma_{1} \Sigma_{3} \Phi, \Sigma_{3} \Phi\right\rangle}{q^{\prime}(\omega)}\left\langle e^{-\mathrm{i} \Sigma_{3} \vartheta} \Sigma_{3} \partial_{\omega} \Phi^{*}, \cdot\right\rangle+\varepsilon_{j}\left\langle\frac{1}{2} \mathrm{i} \beta \alpha_{2} \Sigma_{1} \Sigma_{3} \Phi, \xi_{j}\right\rangle\left\langle e^{-\mathrm{i} \Sigma_{3} \vartheta} \Sigma_{3} \xi_{j}^{*}, \cdot\right\rangle \\
& -\varepsilon_{j}\left\langle\frac{1}{2} \mathrm{i} \beta \alpha_{2} \Sigma_{1} \Sigma_{3} \Phi, \Sigma_{1} C \xi_{j}\right\rangle\left\langle e^{-\mathrm{i} \Sigma_{3} \vartheta} \Sigma_{3} \Sigma_{1}\left(C \xi_{j}\right)^{*}, \cdot\right\rangle+\left\langle e^{-\mathrm{i} \Sigma_{3} \vartheta}\left(P_{c}\left(\mathcal{H}_{\omega}^{*}\right) \frac{1}{2} \mathrm{i} \beta \alpha_{2} \Sigma_{1} \Sigma_{3} \Phi^{*}\right)^{*}, \cdot\right\rangle .
\end{aligned}
$$


By i $\beta \alpha_{2} \Sigma_{1} \Phi=\mathrm{i} \beta \alpha_{2} C \Phi=\left(\mathrm{i} \beta \alpha_{2}\right)^{2} \Phi^{*}=\Phi^{*}$ we have

$$
\left\langle\mathrm{i} \beta \alpha_{2} \Sigma_{3} \Sigma_{1} \Phi, \partial_{\omega} \Phi\right\rangle=\left\langle\phi^{*}, \partial_{\omega} \phi\right\rangle-\left\langle\phi, \partial_{\omega} \phi^{*}\right\rangle=0,
$$

by $\left\langle\phi, \partial_{\omega} \phi^{*}\right\rangle=\int_{\mathbb{R}^{3}}\left(a \partial_{\omega} a+b \partial_{\omega} b\right) d x=\left\langle\phi^{*}, \partial_{\omega} \phi\right\rangle$, see (H:2). Then

$$
\begin{aligned}
& \frac{1}{2}\left\langle e^{-\mathrm{i} \Sigma_{3} \vartheta} \mathrm{i} \beta \alpha_{2} \Sigma_{1} \Sigma_{3} \Phi, \cdot\right\rangle= \\
& -\frac{q}{q^{\prime}}\left\langle e^{-\mathrm{i} \Sigma_{3} \vartheta} \Sigma_{3} \partial_{\omega} \Phi^{*}, \cdot\right\rangle+\varepsilon_{j}\left\langle\frac{1}{2} \mathrm{i} \beta \alpha_{2} \Sigma_{1} \Sigma_{3} \Phi, \xi_{j}\right\rangle\left\langle e^{-\mathrm{i} \Sigma_{3} \vartheta} \Sigma_{3} \xi_{j}^{*}, \cdot\right\rangle \\
& -\varepsilon_{j}\left\langle\frac{1}{2} \mathrm{i} \beta \alpha_{2} \Sigma_{1} \Sigma_{3} \Phi, \Sigma_{1} C \xi_{j}\right\rangle\left\langle e^{-\mathrm{i} \Sigma_{3} \vartheta} \Sigma_{3} \Sigma_{1}\left(C \xi_{j}\right)^{*}, \cdot\right\rangle+\left\langle e^{-\mathrm{i} \Sigma_{3} \vartheta}\left(P_{c}\left(\mathcal{H}_{\omega}^{*}\right) \frac{1}{2} \mathrm{i} \beta \alpha_{2} \Sigma_{1} \Sigma_{3} \Phi^{*}\right)^{*}, \cdot\right\rangle .
\end{aligned}
$$

with by (4.4)

$$
-\frac{q}{q^{\prime}}\left\langle e^{-\mathrm{i} \Sigma_{3} \vartheta} \Sigma_{3} \partial_{\omega} \Phi^{*}, \cdot\right\rangle=\frac{q}{q^{\prime}}\left\langle R, \Sigma_{3} \partial_{\omega}^{2} \Phi^{*}\right\rangle d \omega-\mathrm{i} \frac{q}{q^{\prime}}\left(q^{\prime}+\left\langle R, \partial_{\omega} \Phi^{*}\right\rangle\right) d \vartheta .
$$

Applying Lemma 4.3. we get (by i $\beta \alpha_{2} \Sigma_{1} f=f^{*}$ which follows from $\Sigma_{1} U=C U$ )

$$
\begin{aligned}
& \varpi_{0}=-\mathrm{i} q d \vartheta-\varepsilon_{j} \frac{\bar{z}_{j} d z_{j}-z_{j} d \bar{z}_{j}}{2}+\frac{1}{2}\left\langle f(U), \mathrm{i} \beta \alpha_{2} \Sigma_{3} \Sigma_{1} f^{\prime}(U) \cdot\right\rangle \\
& =\mathrm{i}\left(-q+\frac{1}{2}\|R\|_{L^{2}}^{2}\right) d \vartheta+\frac{1}{2}\left\langle\Sigma_{3} R^{*}, \partial_{\omega} R\right\rangle d \omega++\frac{1}{2}\left\langle\mathrm{i} \beta \alpha_{2} \Sigma_{1} \Sigma_{3}\left(1-P_{c}\left(\omega_{0}\right) P_{c}(\omega)\right) f, f^{\prime}\right\rangle+ \\
& +\frac{1}{2} z_{j}\left\langle e^{-\mathrm{i} \Sigma_{3} \vartheta} \Sigma_{1} \Sigma_{3}\left(C \xi_{j}\right)^{*}, \cdot\right\rangle-\frac{1}{2} \bar{z}_{j}\left\langle e^{-\mathrm{i} \Sigma_{3} \vartheta} \Sigma_{3} \xi_{j}^{*}, \cdot\right\rangle++\frac{1}{2}\left\langle e^{-\mathrm{i} \Sigma_{3} \vartheta} \mathrm{i} \beta \alpha_{2} \Sigma_{1} \Sigma_{3} P_{c}(\omega) f, \cdot\right\rangle .
\end{aligned}
$$

By (4.6) we have

$$
d \psi=\frac{1}{2}\left\langle\Sigma_{3} \Phi^{*}, \partial_{\omega} R\right\rangle d \omega+\frac{1}{2}\left\langle\Sigma_{3} \Phi^{*}, \xi_{j}\right\rangle d z_{j}+\frac{1}{2}\left\langle\Sigma_{3} \Phi^{*}, \Sigma_{1} C \xi_{j}\right\rangle d \bar{z}_{j}+\frac{1}{2}\left\langle\Sigma_{3} \Phi^{*}, P_{c}(\omega) f^{\prime} \cdot\right\rangle
$$

Applying to (7.15) Lemma 4.3 and the identities (7) below, we get $d \psi=$

$$
\begin{aligned}
d \psi & =\frac{1}{2}\left\langle\Sigma_{3} \Phi^{*}, \xi_{j}\right\rangle\left\langle e^{-\mathrm{i} \Sigma_{3} \vartheta} \Sigma_{3} \xi_{j}^{*}, \cdot\right\rangle+\frac{1}{2}\left\langle\Sigma_{3} \Phi^{*}, \Sigma_{1} C \xi_{j}\right\rangle\left\langle e^{-\mathrm{i} \Sigma_{3} \vartheta} \Sigma_{1} \Sigma_{3}\left(C \xi_{j}\right)^{*}, \cdot\right\rangle \\
& +\frac{1}{2}\left\langle e^{-\mathrm{i} \Sigma_{3} \vartheta}\left(P_{c}\left(\mathcal{H}_{\omega}^{*}\right) \Sigma_{3} \Phi\right)^{*}, \cdot\right\rangle \\
& +\frac{q}{q^{\prime}}\left\langle\Sigma_{3} \partial_{\omega} \Phi^{*}, \partial_{\omega} R\right\rangle d \omega \\
& -\frac{\mathrm{i}}{2}\langle\underbrace{\left\langle\Sigma_{3} \Phi^{*}, \xi_{j}\right\rangle \Sigma_{3} \xi_{j}^{*}+\left\langle\Sigma_{3} \Phi^{*}, \Sigma_{1} C \xi_{j}\right\rangle \Sigma_{1} \Sigma_{3}\left(C \xi_{j}\right)^{*}+\left(P_{c}\left(\mathcal{H}_{\omega}^{*}\right) \Sigma_{3} \Phi\right)^{*}}_{\left.P_{N_{g}(\mathcal{H}}\right)^{\Sigma_{3} \Phi^{*}}}, \Sigma_{3} R\rangle d \vartheta .
\end{aligned}
$$

To get the third line of (7.16) we have used:

$$
\begin{aligned}
& \frac{1}{2}\left\langle\Sigma_{3} \Phi^{*}, \partial_{\omega} R\right\rangle-\frac{1}{2}\left\langle\Sigma_{3} \Phi^{*}, \xi_{j}\right\rangle\left\langle\Sigma_{3} \xi_{j}^{*}, \partial_{\omega} R\right\rangle- \\
& \frac{1}{2}\left\langle\Sigma_{3} \Phi^{*}, \Sigma_{1} C \xi_{j}\right\rangle\left\langle\Sigma_{1} \Sigma_{3}\left(C \xi_{j}\right)^{*}, \partial_{\omega} R\right\rangle-\frac{1}{2}\left\langle\left(P_{c}\left(\mathcal{H}_{\omega}^{*}\right) \Sigma_{3} \Phi\right)^{*}, \partial_{\omega} R\right\rangle=\frac{1}{2}\left\langle\Sigma_{3} \Phi^{*}, \partial_{\omega} R\right\rangle ; \\
& -\frac{1}{2}\left[\left\langle\Sigma_{3} \Phi^{*}, \partial_{\omega} R\right\rangle-\frac{1}{q^{\prime}}\left\langle\Sigma_{3} \Phi^{*}, \Sigma_{3} \Phi\right\rangle\left\langle\Sigma_{3} \partial_{\omega} \Phi^{*}, \partial_{\omega} R\right\rangle\right]=\frac{2 q}{2 q^{\prime}}\left\langle\Sigma_{3} \partial_{\omega} \Phi^{*}, \partial_{\omega} R\right\rangle .
\end{aligned}
$$

Let us consider the sum (7.7). There are various cancelations. The first and second (resp. the first term of the third) line of (7.16) cancel with the second and third lines of (7.12) (resp. the first term of the rhs

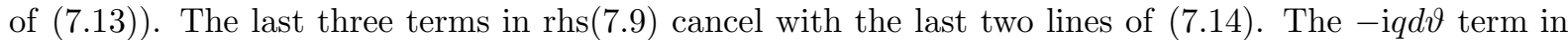
the rhs of (7.14) ) cancels with the $-\mathrm{i} q d \vartheta$ term in (7.13). Adding the fourth line of (7.16) with the last term of rhs(7.13) we get the product of $\mathrm{i}$ times the following quantities: 


$$
\begin{aligned}
& -\frac{1}{2}\left\langle P_{N_{g}^{\perp}\left(\mathcal{H}_{\omega}\right)} \Sigma_{3} \Phi^{*}, \Sigma_{3} R\right\rangle-\frac{q}{q^{\prime}}\left\langle R, \partial_{\omega} \Phi^{*}\right\rangle=-\frac{1}{2}\left\langle\Phi^{*}, R\right\rangle \\
& +\frac{1}{2}\left\langle P_{N_{g}\left(\mathcal{H}_{\omega}^{*}\right)} \Sigma_{3} \Phi^{*}, \Sigma_{3} R\right\rangle-\frac{q}{q^{\prime}}\left\langle R, \partial_{\omega} \Phi^{*}\right\rangle \\
& =-\frac{1}{2}\left\langle\Phi^{*}, R\right\rangle+\frac{1}{2 q^{\prime}}\left\langle\Phi^{*}, \Sigma_{3} R\right\rangle\left\langle\partial_{\omega} \Phi, \Sigma_{3} \Phi^{*}\right\rangle \\
& +\frac{1}{2 q^{\prime}}\left\langle\Sigma_{3} \partial_{\omega} \Phi^{*}, \Sigma_{3} R\right\rangle\left\langle\Sigma_{3} \Phi^{*}, \Sigma_{3} \Phi\right\rangle-\frac{q}{q^{\prime}}\left\langle R, \partial_{\omega} \Phi^{*}\right\rangle=0,
\end{aligned}
$$

where for the second equality we have used

$$
P_{N_{g}\left(\mathcal{H}_{\omega}^{*}\right)}=\frac{1}{q^{\prime}} \Phi^{*}\left\langle\partial_{\omega} \Phi, \cdot\right\rangle+\frac{1}{q^{\prime}} \Sigma_{3} \partial_{\omega} \Phi^{*}\left\langle\Sigma_{3} \Phi, \cdot\right\rangle .
$$

The last equality in (7.17) can be seen as follows. The two terms in the third line in (7.17) are both equal to 0 . Indeed, $\left\langle\Sigma_{3} \Phi^{*}, \partial_{\omega} \Phi\right\rangle=0$ by (7.11) and, by $R \in N_{g}^{\perp}\left(\mathcal{H}_{\omega}^{*}\right)$ and $\Phi^{*} \in N_{g}\left(\mathcal{H}_{\omega}^{*}\right),\left\langle R, \Phi^{*}\right\rangle=0$. The two terms in the fourth line in (7.17) cancel each other. Then we get formulas for $\alpha^{\omega}$ and $\alpha^{f}$. We get $\alpha^{\vartheta}$ also by $\left\|P_{c}(\omega) f\right\|_{2}^{2}=\|f\|_{2}^{2}+2 \Re\left\langle\left(P_{c}(\omega)-P_{c}\left(\omega_{0}\right)\right) f,\left(P_{c}(\omega) f\right)^{*}\right\rangle$.

Lemma 7.3. We have, summing over repeated indexes (also on $j$ and $\bar{j}$ ):

$$
i_{Y} \Omega_{0}=\mathrm{i} q^{\prime} Y_{\vartheta} d \omega-\mathrm{i} q^{\prime} Y_{\omega} d \vartheta+\varepsilon_{j}\left(Y_{j} d \bar{z}_{j}-Y_{\bar{j}} d z_{j}\right)+\left\langle\mathrm{i} \beta \alpha_{2} \Sigma_{1} \Sigma_{3} Y_{f}, f^{\prime} \cdot\right\rangle .
$$

For the $a_{1}$ in (7.4), and for $\Gamma=i_{Y} \widetilde{\Omega}$, we have

$$
\begin{aligned}
\Gamma_{\omega} & =a_{1} Y_{\vartheta}+Y_{j}\left\langle\Sigma_{1} \Sigma_{3}\left(C \xi_{j}\right)^{*}, \partial_{\omega} R\right\rangle-Y_{\bar{j}}\left\langle\Sigma_{3} \xi_{j}^{*}, \partial_{\omega} R\right\rangle+\left\langle Y_{f}, \mathrm{i} \beta \alpha_{2} \Sigma_{3} \Sigma_{1} P_{c} \partial_{\omega} R\right\rangle ; \\
-\Gamma_{\vartheta} & =a_{1} Y_{\omega}-\mathrm{i} Y_{j}\left\langle\Sigma_{1} \Sigma_{3}\left(C \xi_{j}\right)^{*}, \Sigma_{3} R\right\rangle+\mathrm{i} Y_{\bar{j}}\left\langle\Sigma_{3} \xi_{j}^{*}, \Sigma_{3} R\right\rangle-\mathrm{i}\left\langle Y_{f}, \mathrm{i} \beta \alpha_{2} \Sigma_{3} \Sigma_{1} P_{c} \Sigma_{3} R\right\rangle ; \\
-\Gamma_{j} & =\left\langle\Sigma_{1} \Sigma_{3}\left(C \xi_{j}\right)^{*}, \partial_{\omega} R\right\rangle Y_{\omega}+\mathrm{i}\left\langle\Sigma_{1} \Sigma_{3}\left(C \xi_{j}\right)^{*}, \Sigma_{3} R\right\rangle Y_{\vartheta} ; \\
\Gamma_{\bar{j}} & =\left\langle\Sigma_{3} \xi_{j}^{*}, \partial_{\omega} R\right\rangle Y_{\omega}+\mathrm{i}\left\langle\Sigma_{3} \xi_{j}^{*}, \Sigma_{3} R\right\rangle Y_{\vartheta} ; \\
\mathrm{i} \beta \alpha_{2} \Sigma_{3} \Sigma_{1} \Gamma_{f} & =\left(P_{c}\left(\omega_{0}\right) P_{c}(\omega)-1\right) Y_{f}+Y_{\omega} P_{c}\left(\omega_{0}\right) P_{c}(\omega) \partial_{\omega} R+\mathrm{i} Y_{\vartheta} P_{c}\left(\omega_{0}\right) P_{c}(\omega) \Sigma_{3} R .
\end{aligned}
$$

In particular, for $\gamma=i_{Y^{\tau}} \Omega_{\tau}=i_{Y^{\tau}} \Omega_{0}+\tau i_{Y^{\tau}} \widetilde{\Omega}$ we have

$$
\begin{aligned}
\gamma_{\omega}= & \left(\mathrm{i} q^{\prime}+\tau a_{1}\right) Y_{\vartheta}^{\tau}+\tau Y_{j}^{\tau}\left\langle\Sigma_{1} \Sigma_{3}\left(C \xi_{j}\right)^{*}, \partial_{\omega} R\right\rangle-\tau Y_{\bar{j}}^{\tau}\left\langle\Sigma_{3} \xi_{j}^{*}, \partial_{\omega} R\right\rangle \\
& +\tau\left\langle Y_{f}^{\tau}, \mathrm{i} \beta \alpha_{2} \Sigma_{3} \Sigma_{1} P_{c} \partial_{\omega} R\right\rangle ; \\
-\gamma_{\vartheta}= & \left(\mathrm{i} q^{\prime}+\tau a_{1}\right) Y_{\omega}^{\tau}-\tau \mathrm{i} Y_{j}^{\tau}\left\langle\Sigma_{1} \Sigma_{3}\left(C \xi_{j}\right)^{*}, \Sigma_{3} R\right\rangle+\tau \mathrm{i} Y_{j}^{\tau}\left\langle\Sigma_{3} \xi_{j}^{*}, \Sigma_{3} R\right\rangle \\
& -\mathrm{i} \tau\left\langle Y_{f}^{\tau}, \mathrm{i} \beta \alpha_{2} \Sigma_{3} \Sigma_{1} P_{c} \Sigma_{3} R\right\rangle ; \\
-\gamma_{j}= & \varepsilon_{j}\left(Y^{\tau}\right)_{\bar{j}}+\tau\left\langle\Sigma_{1} \Sigma_{3}\left(C \xi_{j}\right)^{*}, \partial_{\omega} R\right\rangle Y_{\omega}^{\tau}+\mathrm{i} \tau\left\langle\Sigma_{1} \Sigma_{3}\left(C \xi_{j}\right)^{*}, \Sigma_{3} R\right\rangle Y_{\vartheta}^{\tau} ; \\
\gamma_{\bar{j}}= & \varepsilon_{j}\left(Y^{\tau}\right)_{j}+\tau\left\langle\Sigma_{3} \xi_{j}^{*}, \partial_{\omega} R\right\rangle Y_{\omega}^{\tau}+\mathrm{i} \tau\left\langle\Sigma_{3} \xi_{j}^{*}, \Sigma_{3} R\right\rangle Y_{\vartheta} ; \\
\mathrm{i} \beta \alpha_{2} \Sigma_{3} \Sigma_{1} \gamma_{f}= & \left(Y^{\tau}\right)_{f}+\tau\left(P_{c}\left(\omega_{0}\right) P_{c}(\omega)-1\right) Y_{f}^{\tau} \\
+ & \tau Y_{\omega}^{\tau} P_{c}\left(\omega_{0}\right) P_{c}(\omega) \partial_{\omega} R+\mathrm{i} \tau Y_{\vartheta}^{\tau} P_{c}\left(\omega_{0}\right) P_{c}(\omega) \Sigma_{3} R .
\end{aligned}
$$

Proof. Identity (7.18) is straightforward. Identity (7.20) follows immediately from (7.18)-(7.19). Finally, (7.19) is elementary linear algebra, and basically the same of [26, Lemma 7.3].

Remark 7.4. Choosing $\gamma=\alpha$ in Lemma 7.3 with $\mathcal{F}_{\tau}$ the flow of $Y^{\tau}$, then $\left(Y^{\tau}\right)_{\vartheta} \not \equiv 0$ is an obstruction to the fact that $K \circ \mathcal{F}_{1}$ is a $\vartheta$ invariant Hamiltonian yielding a semilinear Dirac equation. So we want $\left(Y^{\tau}\right)_{\vartheta}=0$ or $d \vartheta\left(Y^{\tau}\right)=\mathrm{i} \Omega_{\tau}\left(X_{\vartheta}^{\tau}, Y^{\tau}\right)=0$, with $X_{\vartheta}^{\tau}$ the Hamiltonian fields of $\vartheta$. To this effect we add a correction to $\alpha$ and define $Y^{\tau}$ from $\alpha+\mathrm{i} d F$ where $(\alpha+\mathrm{i} d F)\left(X_{\vartheta}^{\tau}\right)=0$.

Lemma 7.5. Consider the vector field $X_{\vartheta}^{\tau}$ (resp. $\left.X_{\omega}^{\tau}\right)$ defined by $i_{X_{\vartheta}^{\tau}} \Omega_{\tau}=-\mathrm{i} d \vartheta$ (resp. $\left.i_{X_{\omega}^{\tau}} \Omega_{\tau}=-\mathrm{i} d \omega\right)$. Then we have (here $P_{c}=P_{c}\left(\mathcal{H}_{\omega}\right)$ and $\left.P_{c}^{0}=P_{c}\left(\mathcal{H}_{\omega_{0}}\right)\right)$ :

$$
\begin{aligned}
X_{\vartheta}^{\tau}= & \left(X_{\vartheta}^{\tau}\right)_{\omega}\left[\frac{\partial}{\partial \omega}-\tau\left\langle\Sigma_{3} \xi_{j}^{*}, \partial_{\omega} R\right\rangle \frac{\partial}{\partial z_{j}}-\tau\left\langle\Sigma_{1} \Sigma_{3}\left(C \xi_{j}\right)^{*}, \partial_{\omega} R\right\rangle \frac{\partial}{\partial \bar{z}_{j}}\right. \\
& \left.-\tau P_{c}^{0}\left(1+\tau P_{c}-\tau P_{c}^{0}\right)^{-1} P_{c}^{0} P_{c} \partial_{\omega} R\right],
\end{aligned}
$$


where $\left(X_{\vartheta}^{\tau}\right)_{\omega}$ is real valued and given by (for the $a_{1}$ in (7.4))

$$
\begin{gathered}
\left(X_{\vartheta}^{\tau}\right)_{\omega}=\frac{\mathrm{i}}{\mathrm{i} q^{\prime}+\tau a_{1}+\tau a_{2}}=-\left(X_{\omega}^{\tau}\right)_{\vartheta} \\
a_{2}:=\mathrm{i} \tau\left\langle\Sigma_{3} \xi_{j}^{*}, \partial_{\omega} R\right\rangle\left\langle\Sigma_{1} \Sigma_{3}\left(C \xi_{j}\right)^{*}, \Sigma_{3} R\right\rangle-\mathrm{i} \tau\left\langle\Sigma_{1} \Sigma_{3}\left(C \xi_{j}\right)^{*}, \partial_{\omega} R\right\rangle\left\langle\xi_{j}^{*}, R\right\rangle+ \\
+\mathrm{i} \tau\left\langle P_{c}^{0}\left(1+\tau P_{c}-\tau P_{c}^{0}\right)^{-1} P_{c}^{0} P_{c} \partial_{\omega} R, \mathrm{i} \beta \alpha_{2} \Sigma_{3} \Sigma_{1} P_{c} \Sigma_{3} R\right\rangle .
\end{gathered}
$$

Proof. The proof is almost the same of [26, Lemma 7.5]. By (7.20) for $\gamma=-\mathrm{i} d \vartheta, X_{\vartheta}^{\tau}$ satisfies

$$
\begin{aligned}
& \left(X_{\vartheta}^{\tau}\right)_{\vartheta}=0 ; \\
& \mathrm{i}=\left(\mathrm{i} q^{\prime}+\tau a_{1}\right)\left(X_{\vartheta}^{\tau}\right)_{\omega}-\mathrm{i} \tau\left\langle\Sigma_{1} \Sigma_{3}\left(C \xi_{j}\right)^{*}, \Sigma_{3} R\right\rangle\left(X_{\vartheta}^{\tau}\right)_{j}+ \\
& +\mathrm{i} \tau\left\langle\Sigma_{3} \xi_{j}^{*}, \Sigma_{3} R\right\rangle\left(X_{\vartheta}^{\tau}\right)_{j}-\mathrm{i} \tau\left\langle\left(X_{\vartheta}^{\tau}\right)_{f}, \mathrm{i} \beta \alpha_{2} \Sigma_{3} \Sigma_{1} P_{c} \Sigma_{3} R\right\rangle ; \\
& \left(X_{\vartheta}^{\tau}\right)_{f}=\tau\left(1-P_{c}^{0} P_{c}\right)\left(X_{\vartheta}^{\tau}\right)_{f}-\tau\left(X_{\vartheta}^{\tau}\right)_{\omega} P_{c}^{0} P_{c} \partial_{\omega} R ; \\
& \left(X_{\vartheta}^{\tau}\right)_{\bar{j}}=-\tau\left(X_{\vartheta}^{\tau}\right)_{\omega}\left\langle\Sigma_{1} \Sigma_{3}\left(C \xi_{j}\right)^{*}, \partial_{\omega} R\right\rangle ;\left(X_{\vartheta}^{\tau}\right)_{j}=-\tau\left(X_{\vartheta}^{\tau}\right)_{\omega}\left\langle\Sigma_{3} \xi_{j}^{*}, \partial_{\omega} R\right\rangle .
\end{aligned}
$$

This yields (7.21) for $X_{\vartheta}^{\tau}$ and the first equality in (7.22). The fact that $\left(X_{\vartheta}^{\tau}\right)_{\omega}$ is real valued follows from (7.22) and the fact that $a_{1}$ and $a_{2}$ are imaginary valued, which can be checked by the definitions.

The following lemma is an immediate consequence of the formulas in Lemma 7.5 and of (7.5).

Lemma 7.6. For any $\left(K^{\prime}, S^{\prime}, K, S\right)$ we have

$$
\begin{aligned}
& \left|1-\left(X_{\vartheta}^{\tau}\right)_{\omega} q^{\prime}\right| \lesssim\|R\|_{H^{-K^{\prime},-S^{\prime}}}^{2} \\
& \left|\left(X_{\vartheta}^{\tau}\right)_{j}\right|+\left|\left(X_{\vartheta}^{\tau}\right)_{\bar{j}}\right|+\left\|\left(X_{\vartheta}^{\tau}\right)_{f}\right\|_{H^{K, S}} \lesssim\|R\|_{H^{-K^{\prime},-S^{\prime}}}
\end{aligned}
$$

Definition 7.7. Set $H_{c}^{K, S}(\omega)=P_{c}(\omega) H^{K, S}$ and denote

$$
\widetilde{\mathcal{P}}^{K, S}=\mathbb{C}^{n} \times H_{c}^{K, S}\left(\omega_{0}\right), \quad \mathcal{P}^{K, S}=\mathbb{R}^{2} \times \widetilde{\mathcal{P}}^{K, S}
$$

with elements $(\vartheta, \omega, z, f) \in \mathcal{P}^{K, S}$ and $(z, f) \in \widetilde{\mathcal{P}}^{K, S}$.

Lemma 7.8. We consider $\forall \tau \in[0,1]$ the hamiltonian field $X_{\vartheta}^{\tau}$ and the flow

$$
\frac{d}{d s} \Phi_{s}(\tau, U)=X_{\vartheta}^{\tau}\left(\Phi_{s}(\tau, U)\right), \Phi_{0}(\tau, U)=U
$$

(1) For any $\left(K^{\prime}, S^{\prime}\right)$ there is a $s_{0}>0$ and a neighborhood $\mathcal{U}$ of $\mathbb{R} \times\left\{\left(\omega_{0}, 0,0\right)\right\}$ in $\mathcal{P}^{-K^{\prime},-S^{\prime}}$ such that the map $(s, \tau, U) \rightarrow \Phi_{s}(\tau, U)$ is smooth

$$
\left(-s_{0}, s_{0}\right) \times[0,1] \times\left(\mathcal{U} \cap\left\{\omega=\omega_{0}\right\}\right) \rightarrow \mathcal{P}^{-K^{\prime},-S^{\prime}} .
$$

(2) $\mathcal{U}$ can be chosen so that for any $\tau \in[0,1]$ there is another neighborhood $\mathcal{V}_{\tau}$ of $\mathbb{R} \times\left\{\left(\omega_{0}, 0,0\right)\right\}$ in $\mathcal{P}^{-K^{\prime},-S^{\prime}}$ s.t. the above map establishes a diffeomorphism

$$
\left(-s_{0}, s_{0}\right) \times\left(\mathcal{U} \cap\left\{\omega=\omega_{0}\right\}\right) \rightarrow \mathcal{V}_{t}
$$

(3) $f\left(\Phi_{s}(\tau, U)\right)-f(U)=G(t, s, z, f)$ is a smooth map for all $(K, S)$

$$
\left(-s_{0}, s_{0}\right) \times[0,1] \times\left(\mathcal{U} \cap\left\{\omega=\omega_{0}\right\}\right) \rightarrow H^{K, S}
$$

with $\|G(t, s, z, f)\|_{H^{K, S}} \leq C|s|\left(|z|+\|f\|_{H^{-K^{\prime},-S^{\prime}}}\right)$.

Proof. The proof is exactly the same of Lemma 7.7 [26]. We only remark, that the field $X_{\vartheta}^{\tau}$, the flow $\Phi_{s}(\tau, U)$ and the function $F(\tau, U)$ in Lemma 7.9 are defined intrinsically, and so are periodic in $\vartheta$. This is because $X_{\vartheta}^{\tau}$ satisfies these properties, since $i_{X_{\vartheta}^{\tau}} \Omega_{\tau}=-\mathrm{i} d \vartheta$ with both $\Omega_{\tau}$ and $d \vartheta$ intrinsically defined and periodic in $\vartheta$.

Lemma 7.9. We consider a scalar function $F(\tau, U)$ defined as follows:

$$
F\left(\tau, \Phi_{s}(\tau, U)\right)=\mathrm{i} \int_{0}^{s} \alpha_{\Phi_{s^{\prime}}(t, U)}\left(X_{\vartheta}^{\tau}\left(\Phi_{s^{\prime}}(t, U)\right)\right) d s^{\prime}, \text { where } \omega(U)=\omega_{0}
$$

We have $F \in C^{\infty}([0,1] \times \mathcal{U}, \mathbb{R})$ for a neighborhood $\mathcal{U}$ of $\mathbb{R} \times\left\{\left(\omega_{0}, 0,0\right)\right\}$ in $\mathcal{P}^{-K^{\prime}},-S^{\prime}$. We have

$$
|F(t, U)| \leq C\left(K^{\prime}, S^{\prime}\right)\left|\omega-\omega_{0}\right|\left(|z|+\|f\|_{H^{-K^{\prime},-S^{\prime}}}\right)^{2}
$$


We have (exterior differentiation only in $U$ )

$$
(\alpha+\mathrm{i} d F)\left(X_{\vartheta}^{\tau}\right)=0 .
$$

Proof. The proof is elementary and is exactly the same of Lemma 7.8 [26].

We now have the desired correction for $\alpha$ and below we introduce the vector field whose flow yields the wanted change of coordinates.

Lemma 7.10. Denote by $\mathcal{X}^{\tau}$ the vector field which solves $i_{\mathcal{X} \tau} \Omega_{\tau}=-\alpha-\mathrm{i} d F(\tau)$. Then the following properties hold.

(1) There is a neighborhood $\mathcal{U}$ of $\mathbb{R} \times\left\{\left(\omega_{0}, 0,0\right)\right\}$ in $\mathcal{P}^{1,0}$ such that $\mathcal{X}^{\tau}(U) \in C^{\infty}\left([0,1] \times \mathcal{U}, \mathcal{P}^{1,0}\right)$.

(2) We have $\left(\mathcal{X}^{\tau}\right)_{\vartheta} \equiv 0$.

(3) For constants $C\left(K, S, K^{\prime}, S^{\prime}\right)$

$$
\begin{aligned}
& \left|\left(\mathcal{X}^{\tau}\right)_{\omega}+\frac{\|f\|_{2}^{2}}{2 q^{\prime}(\omega)}\right| \lesssim\left(|z|+\|f\|_{H^{-K^{\prime},-S^{\prime}}}\right)^{2} ; \\
& \left|\left(\mathcal{X}^{\tau}\right)_{j}\right|+\left|\left(\mathcal{X}^{\tau}\right)_{\bar{j}}\right|+\left\|\left(\mathcal{X}^{\tau}\right)_{f}\right\|_{H^{K, S}} \lesssim\left(|z|+\|f\|_{H^{-K^{\prime},-S^{\prime}}}\right) \times \\
& \times\left(\left|\omega-\omega_{0}\right|+|z|+\|f\|_{H^{-K^{\prime},-S^{\prime}}}+\|f\|_{L^{2}}^{2}\right) .
\end{aligned}
$$

(4) We have $L_{\mathcal{X}^{\tau}} \frac{\partial}{\partial \vartheta}:=\left[\mathcal{X}^{\tau}, \frac{\partial}{\partial \vartheta}\right]=0$.

(5) We have $\left(\mathcal{X}^{\tau}\right)_{\bar{j}}=\overline{\left(\mathcal{X}^{\tau}\right)_{j}},\left(\mathcal{X}^{\tau}\right)_{f}=C \Sigma_{1}\left(\mathcal{X}^{\tau}\right)_{f} \cdot\left(\mathcal{X}^{\tau}\right)_{\omega}$ is real valued.

Proof. The proof is almost the same of [26, Lemma 7.9 ]. Claim (1) follows from the regularity properties of $\alpha, F$ and $\Omega_{\tau}$ and from equations (7.31) and (7.33) below. (7.29) implies (2) by

$$
\mathrm{i}\left(\mathcal{X}^{\tau}\right)_{\vartheta}=\mathrm{i} d \vartheta\left(\mathcal{X}^{\tau}\right)=-i_{X_{\vartheta}^{\tau}} \Omega_{\tau}\left(\mathcal{X}^{\tau}\right)=i_{\mathcal{X}^{\tau}} \Omega_{\tau}\left(X_{\vartheta}^{\tau}\right)=-(\alpha+\mathrm{i} d F)\left(X_{\vartheta}^{\tau}\right)=0 .
$$

We have $\mathrm{i}\left(\mathcal{X}^{\tau}\right)_{\omega}=\mathrm{i} d \omega\left(\mathcal{X}^{\tau}\right)=-i_{X_{\omega}^{\tau}} \Omega_{\tau}\left(\mathcal{X}^{\tau}\right)$, so

$$
\begin{aligned}
& \mathrm{i}\left(\mathcal{X}^{\tau}\right)_{\omega}=i_{\mathcal{X} \tau} \Omega_{\tau}\left(X_{\omega}^{\tau}\right)=-\left(X_{\omega}^{\tau}\right)_{\vartheta}\left[\alpha^{\vartheta}+\tau \partial_{j} F\left\langle\xi_{j}^{*}, R\right\rangle-\tau \partial_{\bar{j}} F\left\langle\Sigma_{1}\left(C \xi_{j}\right)^{*}, R\right\rangle\right. \\
& \left.+\tau\left\langle\nabla_{f} F+\mathrm{i} \alpha^{f}, P_{c}^{0}\left(1+\tau P_{c}-\tau P_{c}^{0}\right)^{-1} P_{c}^{0} P_{c} \Sigma_{3} R\right\rangle\right] .
\end{aligned}
$$

Then by (7.8), (7.22) and (7.23), we get the first inequality in (7.30):

$$
\left|\left(\mathcal{X}^{\tau}\right)_{\omega}+\frac{\|f\|_{2}^{2}}{2 q^{\prime}(\omega)}\right| \leq C\left(|z|+\|f\|_{H^{-K^{\prime},-S^{\prime}}}\right)^{2} .
$$

By (7.20) we have the following equations

$$
\begin{aligned}
\mathrm{i} \partial_{j} F & =\varepsilon_{j}\left(\mathcal{X}^{\tau}\right)_{j}+\tau\left\langle\Sigma_{1} \Sigma_{3}\left(C \xi_{j}\right)^{*}, \partial_{\omega} R\right\rangle\left(\mathcal{X}^{\tau}\right)_{\omega} \\
-\mathrm{i} \partial_{\bar{j}} F & =\varepsilon_{j}\left(\mathcal{X}^{\tau}\right)_{j}+\tau\left\langle\Sigma_{3} \xi_{j}^{*}, \partial_{\omega} R\right\rangle\left(\mathcal{X}^{\tau}\right)_{\omega} \\
\mathrm{i} \beta \alpha_{2} \Sigma_{3} \Sigma_{1}\left(\alpha^{f}+\mathrm{i} \nabla_{f} F\right) & =-\left(\mathcal{X}^{\tau}\right)_{f}-\tau\left(P_{c}^{0} P_{c}-1\right)\left(\mathcal{X}^{\tau}\right)_{f}-\tau\left(\mathcal{X}^{\tau}\right)_{\omega} P_{c}^{0} P_{c} \partial_{\omega} R .
\end{aligned}
$$

Formulas (7.33) imply

$$
\begin{aligned}
& \left|\left(\mathcal{X}_{\omega}^{\tau}\right)_{\bar{j}}\right| \leq\left|\partial_{j} F\right|+C\left(|z|+\|f\|_{H^{-K^{\prime},-S^{\prime}}}\right)\left|\left(\mathcal{X}^{\tau}\right)_{\omega}\right| \\
& \left|\left(\mathcal{X}_{\omega}^{\tau}\right)_{j}\right| \leq\left|\partial_{\bar{j}} F\right|+C\left(|z|+\|f\|_{H^{-K^{\prime},-S^{\prime}}}\right)\left|\left(\mathcal{X}^{\tau}\right)_{\omega}\right| \\
& \left\|\left(\mathcal{X}_{\omega}^{\tau}\right)_{f}\right\|_{H^{K, S}} \leq\left\|\alpha^{f}\right\|_{H^{K, S}}+\left\|\nabla_{f} F\right\|_{H^{K, S}}+C\left(|z|+\|f\|_{H^{-K^{\prime},-S^{\prime}}}\right)\left|\left(\mathcal{X}^{\tau}\right)_{\omega}\right|
\end{aligned}
$$

which with (7.32), (7.8) and Lemma (17.28) imply (7.30). Claim (4) follows by $L_{\frac{\partial}{\partial \vartheta}}(\alpha+\mathrm{i} d F)=0$ and by the product rule for the Lie derivative,

$$
L_{\frac{\partial}{\partial \vartheta}}\left(i_{\mathcal{X} \tau} \Omega_{\tau}\right)=i_{\left[\frac{\partial}{\partial \vartheta}, \mathcal{X}^{\tau}\right]} \Omega_{\tau}+i_{\mathcal{X} \tau} L_{\frac{\partial}{\partial \vartheta}} \Omega_{\tau}=i_{\left[\frac{\partial}{\partial \vartheta}, \mathcal{X}^{\tau}\right]} \Omega_{\tau} .
$$

It is elementary to check that (7.31) and (7.33) imply Claim (6), when we use the fact that $\left(X_{\omega}^{\tau}\right)_{\vartheta}$ is real valued, we consider (17.8), the fact that $F$ is real valued.

The following lemma gathers some properties of the change of coordinates. 
Lemma 7.11. Consider the vectorfield $\mathcal{X}^{\tau}$ in Lemma 7.9 and denote by $\mathcal{F}_{\tau}(U)$ the corresponding flow. Then the flow $\mathcal{F}_{\tau}(U)$ for $U$ near $e^{\mathrm{i} \Sigma_{3} \vartheta} \Phi_{\omega_{0}}$ is defined for all $\tau \in[0,1]$. We have $\vartheta \circ \mathcal{F}_{1}=\vartheta$. We have

$$
\begin{aligned}
& q\left(\omega\left(\mathcal{F}_{1}(U)\right)\right)=q(\omega(U))-\frac{\|f\|_{2}^{2}}{2}+\mathcal{E}_{\omega}(U) \\
& z_{j}\left(\mathcal{F}_{1}(U)\right)=z_{j}(U)+\mathcal{E}_{j}(U) \\
& f\left(\mathcal{F}_{1}(U)\right)=f(U)+\mathcal{E}_{f}(U)
\end{aligned}
$$

with

$$
\begin{gathered}
\left|\mathcal{E}_{\omega}(U)\right| \lesssim\left(\left|\omega-\omega_{0}\right|+|z|+\|f\|_{H^{-K^{\prime},-S^{\prime}}}\right)^{2}, \\
\left|\mathcal{E}_{j}(U)\right|+\left\|\mathcal{E}_{f}(U)\right\|_{H^{K, S}} \lesssim\left(\left|\omega-\omega_{0}\right|+|z|+\|f\|_{H^{-K^{\prime},-S^{\prime}}}+\|f\|_{L^{2}}^{2}\right) \\
\times\left(\left|\omega-\omega_{0}\right|+|z|+\|f\|_{H^{-K^{\prime},-S^{\prime}}}\right) .
\end{gathered}
$$

For each $\zeta=\omega, z_{j}, f$ we have $\mathcal{E}_{\zeta}(U)=\mathcal{E}_{\zeta}\left(\|f\|_{L^{2}}^{2}, \omega, z, f\right)$ with, for a neighborhood $\mathcal{U}^{-K^{\prime},-S^{\prime}}$ of $\left\{\left(\omega_{0}, 0,0\right)\right\}$ in $\mathcal{P}^{-K^{\prime},-S^{\prime}} \cap\{\vartheta=0\}$ and for some fixed $a_{0}>0$

$$
\begin{gathered}
\mathcal{E}_{\zeta}(\varrho, \omega, z, f) \in C^{\infty}\left(\left(-a_{0}, a_{0}\right) \times \mathcal{U}^{-K^{\prime},-S^{\prime}}, \mathbb{C}\right) \text { for } \zeta=\omega, z_{j} \\
\mathcal{E}_{f}(\varrho, \omega, z, f) \in C^{\infty}\left(\left(-a_{0}, a_{0}\right) \times \mathcal{U}^{-K^{\prime},-S^{\prime}}, H^{K, S} \cap \mathbf{X}\right) .
\end{gathered}
$$

Proof. The argument is the same of Lemma 7.10 [26], but we review it for the sake of the reader. We add a new variable $\varrho$. We define a new field by

$$
\begin{aligned}
& \mathrm{i}\left(Y^{\tau}\right)_{\omega}=-\left(X_{\omega}^{\tau}\right)_{\vartheta}\left[\alpha^{\vartheta}+\mathrm{i} \frac{\|f\|_{2}^{2}-\rho}{2}+\tau \partial_{j} F\left\langle\xi_{j}^{*}, R\right\rangle-\tau \partial_{\bar{j}} F\left\langle\Sigma_{1}\left(C \xi_{j}\right)^{*}, R\right\rangle\right. \\
& \left.+\tau\left\langle\nabla_{f} F+\mathrm{i} \alpha^{f}, P_{c}^{0}\left(1+\tau P_{c}-\tau P_{c}^{0}\right)^{-1} P_{c}^{0} P_{c} \Sigma_{3} R\right\rangle\right],
\end{aligned}
$$

which implies that $\left(Y^{\tau}\right)_{\omega}$ is real valued, by

$$
\begin{aligned}
\mathrm{i} \partial_{j} F & =\varepsilon_{j}\left(Y^{\tau}\right)_{\bar{j}}+\tau\left\langle\Sigma_{1} \Sigma_{3}\left(C \xi_{j}\right)^{*}, \partial_{\omega} R\right\rangle\left(Y^{\tau}\right)_{\omega} \\
-\mathrm{i} \partial_{j} F & =\varepsilon_{j}\left(Y^{\tau}\right)_{j}+\tau\left\langle\Sigma_{3} \xi_{j}^{*}, \partial_{\omega} R\right\rangle\left(Y^{\tau}\right)_{\omega} \\
\mathrm{i} \beta \alpha_{2} \Sigma_{3} \Sigma_{1}\left(\alpha^{f}+\mathrm{i} \nabla_{f} F\right) & =-\left(Y^{\tau}\right)_{f}-\tau\left(P_{c}^{0} P_{c}-1\right)\left(Y^{\tau}\right)_{f}-\tau\left(Y^{\tau}\right)_{\omega} P_{c}^{0} P_{c} \partial_{\omega} R
\end{aligned}
$$

where we see $\left(Y^{\tau}\right)_{\bar{j}}={\overline{\left(Y^{\tau}\right)}}_{j}, C \Sigma_{1}\left(Y^{\tau}\right)_{f}=\left(Y^{\tau}\right)_{f}$ and $\left(Y^{\tau}\right)_{f} \in X_{c}\left(\mathcal{H}_{\omega_{0}}\right)$, and by $Y_{\rho}^{\tau}=2\left\langle\left(Y^{\tau}\right)_{f}, \mathrm{i} \beta \alpha_{2} \Sigma_{1} f\right\rangle$. Then $Y^{\tau}=Y^{\tau}(\omega, \rho, z, f)$ defines a new flow $\mathcal{G}_{\tau}(\rho, U)$, which reduces to $\mathcal{F}_{\tau}(U)$ in the invariant manifold defined by $\rho=\|f\|_{2}^{2}$. Notice that by $\rho(t)=\rho(0)+\int_{0}^{t} Y_{\rho}^{s} d s$ it is easy to conclude $\rho\left(\mathcal{G}_{1}(\rho, U)\right)=\rho(U)+$ $O(\operatorname{rhs}(7.35))$. Using (7.8), (7.22), (7.25) and (7.39) it is then easy to get

$$
q(\omega(t))=q(\omega(0))+\int_{0}^{t} q^{\prime}(\omega(s)) Y_{\omega}^{s} d s=q(\omega(0))-\int_{0}^{t} \frac{\rho(s)}{2} d s+O(\operatorname{rhs}(\overline{7.35}) .
$$

By standard arguments, see for example the proof of Lemma 4.3 [2], we get

$$
\begin{aligned}
& q\left(\omega\left(\mathcal{G}_{1}(\rho, U)\right)\right)=q(\omega(U))-\frac{\rho}{2}+\mathcal{E}_{\omega}(\rho, U) \\
& z_{\ell}\left(\mathcal{G}_{1}(\rho, U)\right)=z_{\ell}(U)+\mathcal{E}_{\ell}(\rho, U) \\
& f\left(\mathcal{G}_{1}(\rho, U)\right)=f(U)+\mathcal{E}_{f}(\rho, U)
\end{aligned}
$$

with $\mathcal{E}_{\zeta}(\rho, U)$ satisfying (7.37) for $\zeta=\omega, z_{\ell}$, (7.38) for $\zeta=f$ and such that $C \Sigma_{1} \mathcal{E}_{f}(\rho, U)=\mathcal{E}_{f}(\rho, U)$. We have $\mathcal{E}_{\zeta}(U)=\mathcal{E}_{\zeta}\left(\|f\|_{2}, U\right)$ satisfying (7.35) for $\zeta=\omega$ and (7.36) for $\zeta=z_{\ell}, f$.

Eventually we have the desired Darboux type result:

Lemma 7.12. (Darboux Theorem) Consider the flow $\mathcal{F}_{\tau}$ of Lemma 7.11. Then we have $\mathcal{F}_{\tau}^{*} \Omega_{\tau}=\Omega_{0}$. We have $Q \circ \mathcal{F}_{1}=q$. If $\chi$ is a function with $\partial_{\vartheta} \chi \equiv 0$, then $\partial_{\vartheta}\left(\chi \circ \mathcal{F}_{t}\right) \equiv 0$.

Proof. The proof is the same of Lemma 7.11 [26]. 


\section{ReFormulation of (6.6) IN THE NEW COORDinAtes}

We set $H:=K \circ \mathcal{F}_{1}$. In the new coordinates (6.6) becomes

$$
\begin{gathered}
q^{\prime} \dot{\omega}=\frac{\partial H}{\partial \vartheta} \equiv 0, \quad q^{\prime} \dot{\vartheta}=-\frac{\partial H}{\partial \omega}, \\
\mathrm{i} \dot{z}_{j}=\varepsilon_{j} \frac{\partial H}{\partial \bar{z}_{j}}, \quad \mathrm{i} \dot{f}=\mathrm{i} \beta \alpha_{2} \Sigma_{3} \Sigma_{1} \nabla_{f} H .
\end{gathered}
$$

Recall that we are solving the initial value problem (1.1) and that we have chosen $\omega_{0}$ with $q\left(\omega_{0}\right)=\left\|u_{0}\right\|_{L_{x}^{2}}^{2}$. Correspondingly it is enough to focus on (8.2) with $\omega=\omega_{0}$. Consider the notation of Theorem 1.3. Let us focus for the moment on the case $\varepsilon_{j} \equiv 1$ in system (8.2). Then we prove :

Theorem 8.1. Assume $\left(\mathbf{H : 1 )}(\mathbf{H : 1 2})\right.$. Then for any integer $k_{0}>3$ there exist $\epsilon_{0}>0$ and $C>0$ such that for $|z(0)|+\|f(0)\|_{H^{k_{0}}} \leq \epsilon<\epsilon_{0}$ the corresponding solution of (8.2) is globally defined and there are $f_{ \pm} \in H^{k_{0}}$ with $\left\|f_{ \pm}\right\|_{H^{k_{0}}} \leq C \epsilon$ such that

$$
\lim _{t \rightarrow \pm \infty}\left\|e^{\mathrm{i} \vartheta(t) \Sigma_{3}} f(t)-e^{-\mathrm{i} t D_{m}} f_{ \pm}\right\|_{H^{k_{0}}}=0
$$

and $\lim _{t \rightarrow \infty} z(t)=0$, for $\vartheta(t)$ the exponent in (4.1). Fix $p_{0}>2$ and $\tau_{0}>1$. Let $\frac{1}{p}=\frac{1}{2}-\frac{1}{q}$ and $\alpha(q)=\frac{3}{p}$. Then, we can choose $\epsilon_{0}$ small enough such that $f(t, x)=A(t, x)+\widetilde{f}(t, x)$ with

$$
\forall n \in \mathbb{N}, C_{n}(t):=\sup _{x \in \mathbb{R}^{3}}\langle x\rangle^{n}|A(t, x)| \rightarrow 0 \text { as } t \rightarrow \infty
$$

and for some fixed $C$

$$
\|\tilde{f}\|_{L_{t}^{p}\left([0, \infty), B_{q, 2}^{k_{0}-\frac{3}{p}}\right) \cap L_{t}^{2}\left([0, \infty), H_{x}^{k_{0},-\tau_{0}}\right) \cap L_{t}^{2}\left([0, \infty), L_{x}^{\infty}\right)} \leq C \epsilon .
$$

There exist $\omega_{+}$such that $\left|\omega_{+}-\omega_{0}\right|=O\left(\left\|f_{+}\right\|_{2}^{2}\right)$ such that $\lim _{t \rightarrow+\infty} \omega(t)=\omega_{+}$.

Proof that Theorem [8.1 implies Theorem 1.3. If we denote $\left(\omega, z^{\prime}, f^{\prime}\right)$ the initial coordinates, and $\left(\omega_{0}, z, f\right)$ the coordinates in (8.2), we have from Lemma 7.11 :

$$
\left|z^{\prime}-z\right|=O\left(|z|+\|f\|_{L_{x}^{2,-2}}\right) \text { and }\left\|f^{\prime}-f\right\|_{H^{K, S}}=O\left(|z|+\|f\|_{L_{x}^{2,-2}}\right)
$$

for any $(K, S) \in\left(\mathbb{R}^{+}\right)^{2}$. The two error terms $O$ converge to 0 as $t \rightarrow \infty$. Hence the asymptotic behavior of $\left(z^{\prime}, f^{\prime}\right)$ and of $(z, f)$ is the same. We also have, from Lemma 7.11 $q(\omega(t))=q\left(\omega_{0}\right)-\frac{\|f(t)\|_{2}^{2}}{2}+O(|z(t)|+$ $\|f(t)\|_{\left.L_{x}^{2,-2}\right)}$ which implies, say at $+\infty$

$$
\lim _{t \rightarrow+\infty} q(\omega(t))=\lim _{t \rightarrow+\infty}\left(q\left(\omega_{0}\right)-\frac{\left\|e^{-\mathrm{i} t \mathcal{H}_{\omega_{0}, 0}} f_{+}\right\|_{2}^{2}}{2}\right)=q\left(\omega_{0}\right)-\frac{\left\|f_{+}\right\|_{2}^{2}}{2}=q\left(\omega_{+}\right)
$$

for $\omega_{+}$the unique element near $\omega_{0}$ for which the last inequality holds. So $\lim _{t \rightarrow+\infty} \omega(t)=\omega_{+}$.

In the case $\varepsilon_{j} \in\{1,-1\}$ with $\varepsilon_{j} \not \equiv 1$, using the same argument of Theorem 8.1 we prove that solutions which remain close to the standing wave, actually have remainder which scatters. We state this in terms of the system (8.2) and the coordinates after Darboux, but of course it can be stated also in terms of the original coordinates, as in Theorems 1.3 and 1.10

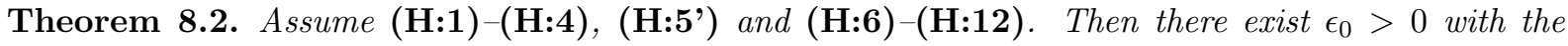
following property. Suppose that $(z(t), f(t))$ is a solution of (18.2) such that $|z(t)|+\|f(0)\|_{H^{k_{0}}} \leq \epsilon<\epsilon_{0}$ for all $t \geq 0$. Suppose furthermore that there exists a fixed $C>0$ such that $\|f(t)\|_{H^{k_{0}}} \leq C \epsilon$ for all $t \geq 0$. Then there exist $f_{+} \in H^{k_{0}}$ such that (8.3) holds (case + ) and we have $\lim _{t \rightarrow+\infty} z(t)=0$. Furthermore, we can write $f(t, x)=A(t, x)+\widetilde{f}(t, x)$ as in Theorem 8.1 in such a way that the same conclusions of Theorem 8.1 regarding $A(t, x)$ and $\tilde{f}$ hold.

Remark 8.3. Theorem 8.2 is analogous to an observation in 43 . regarding the fact that solutions remaining for all times close to a standing wave, stable or unstable, converge to it. Among other references see also [5, 44].

Finally, Theorem 1.10, that is orbital instability, is a consequence of the following theorem.

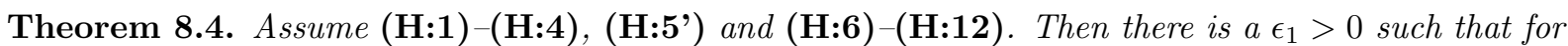
any $\delta>0$ there is a solution $(z(t), f(t))$ of (8.2) such that $|z(0)|+\|f(0)\|_{H^{k_{0}}} \leq \delta$ but there exists $t \geq 0$ such that $|z(t)| \geq \epsilon_{1}$. 
8.1. Taylor expansions. We recall that $\varepsilon_{j}=\left\langle\xi_{j}, \Sigma_{3} \xi_{j}\right\rangle \in\{1,-1\}$ is the signature of the eigenvalues of $\mathcal{H}_{\omega}$. We set $d(\omega):=E\left(\Phi_{\omega}\right)+\omega Q\left(\Phi_{\omega}\right)$. We recall that $\omega_{0}$ is the unique element such that $q\left(\omega_{0}\right)=\left\|u_{0}\right\|_{2}^{2}$ and $G$ is the primitive of the non-linearity $g$ vanishing at 0 .

Lemma 8.5. The following statements hold.

$$
\begin{aligned}
& K=d(\omega)-\omega\left\|u_{0}\right\|_{2}^{2}+K_{2}+K_{P} \text { with } \\
& K_{2}:=\sum_{j} \varepsilon_{j} \lambda_{j}(\omega)\left|z_{j}\right|^{2}+\frac{1}{2}\left\langle i \beta \alpha_{2} \Sigma_{1} \Sigma_{3} \mathcal{H}_{\omega} f, f\right\rangle \text { and } \\
& K_{P}=\left\langle G_{6}(\omega, f(x)), 1\right\rangle+\sum_{|\mu+\nu|=3}\left\langle k_{\mu \nu}(\omega, z), 1\right\rangle z^{\mu} \bar{z}^{\nu}+\sum_{|\mu+\nu|=2} z^{\mu} \bar{z}^{\nu}\left\langle K_{\mu \nu}(\omega, z), \mathrm{i} \beta \alpha_{2} \Sigma_{3} \Sigma_{1} P_{c}(\omega) f\right\rangle \\
& +\sum_{d=2}^{4}\left\langle G_{d}(\omega, z),\left(P_{c}(\omega) f\right)^{\otimes d}\right\rangle+\int_{\mathbb{R}^{3}}\left\langle G_{5}(x, \omega, z, f(x)), f^{\otimes 5}(x)\right\rangle d x,
\end{aligned}
$$

where for a small neighborhood $\mathcal{U}$ of $\left(\omega_{0}, 0\right)$ in $\mathcal{O} \times \mathbb{C}^{n}$, we have what follows.

(1) $G_{6}(x, \omega, f)=G\left(\frac{1}{2}\left(P_{c}(\omega) f(x)\right) \cdot i \alpha_{2} \Sigma_{3} \Sigma_{1}\left(P_{c}(\omega) f(x)\right)\right)$,

(2) $k_{\mu \nu}(\cdot, \omega, z) \in C^{\infty}\left(\mathcal{U}, H_{x}^{K, S}\left(\mathbb{R}^{3}, \mathbb{C}^{8}\right)\right.$,

(3) $K_{\mu \nu}(\cdot, \omega, z) \in C^{\infty}\left(\mathcal{U}, H_{x}^{K, S}\left(\mathbb{R}^{3}, \mathbb{C}^{8}\right) \cap \mathbf{X}\right)$,

(4) $G_{d}(\cdot, \omega, z) \in C^{\infty}\left(\mathcal{U}, H_{x}^{K, S}\left(\mathbb{R}^{3}, B\left(\left(\mathbb{C}^{8}\right)^{\otimes d}, \mathbb{C}\right)\right)\right)$, for $2 \leq d \leq 4$ and $G_{2}(\cdot, \omega, 0) \equiv 0$.

(5) Let $^{t} \eta=(\zeta, C \zeta)$ for $\zeta \in \mathbb{C}^{4}$. Then for $G_{5}(\cdot, \omega, z, \eta)$ we have

$$
\forall l \in \mathbb{N} \cup\{0\},\left\|\nabla_{\omega, z, \bar{z}, \zeta, C \zeta}^{l} G_{5}(\omega, z, \eta)\right\|_{H_{x}^{K, S}\left(\mathbb{R}^{3}, B\left(\left(\mathbb{C}^{8}\right)^{\otimes 5}, \mathbb{C}\right)\right.} \leq C_{l} .
$$

(6) We have $k_{\mu \nu}=k_{\nu \mu}^{*}, K_{\mu \nu}=-C \Sigma_{1} K_{\nu \mu}$.

Proof. Consider $U=e^{\mathrm{i} \Sigma_{3} \vartheta}\left(\Phi_{\omega}+R\right)$ as in (4.1). Decompose $R$ as in (4.5). Set $U=\varphi(\omega, z)+P_{c}(\omega) f$. Let $K_{p}(U)=\int h(U(x)) d x$, see Lemma 2.4, then after first a Taylor integral expansion around $f$ at first order and a Taylor integral expansion around $\phi$ at fourth order, we have

$$
\begin{aligned}
h(U) & =h\left(P_{c}(\omega) f\right)+\int_{0}^{1} d h\left(t \varphi+P_{c}(\omega) f\right) \varphi d t \\
& =h\left(P_{c}(\omega) f\right)+\int_{0}^{1} \sum_{i \leq 4} \frac{1}{i !} d^{i+1} h(t \varphi)\left(P_{c}(\omega) f\right)^{i} \varphi d t+ \\
& +5 \int_{[0,1]^{2}}(1-s)^{4} \frac{1}{5 !} d^{6} h\left(t \varphi+s P_{c}(\omega) f\right)\left(P_{c}(\omega) f\right)^{4} \varphi d t d s
\end{aligned}
$$

Since $\Phi_{\omega}$ is a critical point of $K$ as it is in the kernel of $\mathcal{H}_{\omega} \Sigma_{3}$, so in the Taylor expansion of $K$ around $\Phi_{\omega}$ there is no first order term. The second derivative of $K$ is the bilinear form $\frac{1}{2}\left\langle\mathrm{i} \beta \alpha_{2} \Sigma_{1} \Sigma_{3} \mathcal{H}_{\omega} \cdot, \cdot\right\rangle$. This gives $K_{2}$.

The term $K_{P}$ contains all terms of order higher than 2 in $f$ and $z$. Thus coincides with the term of order higher than 2 in $f$ and $z$ in the above expansion after integration in $x$.

The Hamiltonian $K$ is a real quantity and considering its conjugate will exchange $\bar{z}$ and $z$ and lead by a straightforward calculation to the last assertion. The fact that $K_{\mu \nu}(\omega, z) \in \mathbf{X}$ follows from $\left\langle\mathrm{i} \beta \alpha_{2} \Sigma_{1} \Sigma_{3} \mathbf{A} X, Y\right\rangle=\left\langle\mathrm{i} \beta \alpha_{2} \Sigma_{1} \Sigma_{3} X, \mathbf{A} Y\right\rangle,\left\langle\mathrm{i} \beta \alpha_{2} \Sigma_{1} \Sigma_{3} \mathbf{B} X, Y\right\rangle=\left\langle\mathrm{i} \beta \alpha_{2} \Sigma_{1} \Sigma_{3} X, \mathbf{B} Y\right\rangle$ and $f \in \mathbf{X}$, see Lemma 2.3 .

The following lemma is a reformulation with some rearrangements of the above one in the canonical coordinates provided by Lemma 7.11 . We set $\delta_{j}$ be for $j \in\{1, \ldots n\}$ the multi index $\delta_{j}=\left(\delta_{1 j}, \ldots, \delta_{n j}\right)$. Let $\lambda_{j}^{0}=\lambda_{j}\left(\omega_{0}\right)$ and $\lambda^{0}=\left(\lambda_{1}^{0}, \cdots, \lambda_{n}^{0}\right)$.

Lemma 8.6. Let $H:=K \circ \mathcal{F}_{1}$. Then, around $e^{i \Sigma_{3} \vartheta} \Phi_{\omega_{0}}$ we have the expansion

$$
\begin{gathered}
H=d\left(\omega_{0}\right)-\omega_{0}\left\|u_{0}\right\|_{2}^{2}+\psi\left(\|f\|_{2}^{2}\right)+H_{2}^{(1)}+\mathcal{R}^{(1)}, \text { where } \\
H_{2}^{(1)}=\sum_{\substack{|\mu+\nu|=2 \\
\lambda^{0} \cdot(\mu-\nu)=0}} k_{\mu \nu}^{(1)}\left(\|f\|_{2}^{2}\right) z^{\mu} \bar{z}^{\nu}+\frac{1}{2}\left\langle\mathrm{i} \beta \alpha_{2} \Sigma_{1} \Sigma_{3} \mathcal{H}_{\omega_{0}} f, f\right\rangle .
\end{gathered}
$$


and $\mathcal{R}^{(1)}=\widetilde{\mathcal{R}^{(1)}}+\widetilde{\mathcal{R}^{(2)}}$, with

$(8.8)$

$$
\begin{aligned}
& \widetilde{\mathcal{R}^{(1)}}=\sum_{\substack{|\mu+\nu|=2 \\
\lambda^{0} \cdot(\mu-\nu) \neq 0}} k_{\mu \nu}^{(1)}\left(\|f\|_{2}^{2}\right) z^{\mu} \bar{z}^{\nu}+\sum_{|\mu+\nu|=1} z^{\mu} \bar{z}^{\nu}\left\langle H_{\nu \mu}\left(\|f\|_{2}^{2}\right), \mathrm{i} \beta \alpha_{2} \Sigma_{3} \Sigma_{1} f\right\rangle \\
& \widetilde{\mathcal{R}^{(2)}}=\int_{\mathbb{R}^{3}} G\left(\frac{1}{2}\left(P_{c}\left(\omega_{0}\right) f(x)\right) \cdot i \alpha_{2} \Sigma_{3} \Sigma_{1}\left(P_{c}\left(\omega_{0}\right) f(x)\right)\right) d x+\sum_{|\mu+\nu|=3} z^{\mu} \bar{z}^{\nu} \int_{\mathbb{R}^{3}} k_{\mu \nu}\left(x, z, f, f(x),\|f\|_{2}^{2}\right) d x \\
& +\sum_{|\mu+\nu|=2} z^{\mu} \bar{z}^{\nu} \int_{\mathbb{R}^{3}}\left[\mathrm{i} \beta \alpha_{2} \Sigma_{1} \Sigma_{3} H_{\nu \mu}\left(x, z, f, f(x),\|f\|_{2}^{2}\right)\right]^{T} f(x) d x \\
& +\sum_{j=2}^{5} \mathcal{R}_{j}^{(1)}+\widehat{\mathcal{R}}_{2}^{(1)}\left(z, f,\|f\|_{2}^{2}\right) \\
& \text { and } \mathcal{R}_{j}^{(1)}=\int_{\mathbb{R}^{3}} F_{j}\left(x, z, f, f(x),\|f\|_{2}^{2}\right) f^{\otimes j}(x) d x
\end{aligned}
$$

and where the following holds.

(1) We have $\psi(s)$ is smooth with $\psi(0)=\psi^{\prime}(0)=0$.

(2) At $\|f\|_{2}=0$ we have:

$k_{\mu \nu}^{(1)}(0)=0$ for $|\mu+\nu|=2$ with $(\mu, \nu) \neq\left(\delta_{j}, \delta_{j}\right)$ for all $j ;$

$$
\begin{aligned}
& k_{\delta_{j} \delta_{j}}^{(1)}(0)=\varepsilon_{j} \lambda_{j}\left(\omega_{0}\right), \text { where } \delta_{j}=\left(\delta_{1 j}, \ldots, \delta_{m j}\right) \text { and here we are not summing in } j ; \\
& H_{\nu \mu}(0)=0 \text { for }|\mu+\nu|=1 \text {. }
\end{aligned}
$$

These $k_{\mu \nu}^{(1)}(\varrho)$ and $H_{\nu \mu}(x, \varrho)$ are smooth in all variables with $H_{\nu \mu}(\cdot, \cdot) \in C^{\infty}\left(\mathbb{R}_{\varrho}, H_{x}^{K, S}\left(\mathbb{R}^{3}, \mathbb{C}^{8}\right) \cap \mathbf{X}\right)$ for all $(K, S)$.

(3) We have for all indexes

$$
k_{\mu \nu}^{(1)}=\left(k_{\mu \nu}^{(1)}\right)^{*}, \quad k_{\mu \nu}=k_{\mu \nu}^{*}, \quad H_{\nu \mu}=-C \Sigma_{1} H_{\mu \nu} .
$$

(4) We have $F_{2}(x, 0,0,0,0)=0$.

(5) For all $\left(K, S, K^{\prime}, S^{\prime}\right)$ positives there is a neighborhood $\mathcal{U}^{-K^{\prime},-S^{\prime}}$ of $\{(0,0)\}$ in $\widetilde{\mathcal{P}}^{-K^{\prime},-S^{\prime}}$, see (7.26), such that

(a) for ${ }^{t} \eta=(\zeta, C \zeta)$ where $\zeta \in \mathbb{C}^{4}$. we have, for $k_{\mu \nu}(x, z, f, \eta, \varrho)$ with $(z, f, \zeta, \varrho) \in \mathcal{U}^{-K^{\prime},-S^{\prime}} \times$ $\mathbb{C}^{4} \times \mathbb{R}$

$$
\forall l \in \mathbb{N}^{6}, \quad\left\|\nabla_{z, \bar{z}, \zeta, C \zeta, f, \varrho}^{l} k_{\mu \nu}\right\|_{H_{x}^{K, S}\left(\mathbb{R}^{3}, \mathbb{C}\right)} \leq C_{l} ;
$$

(b) for $H_{\nu \mu}(x, z, f, g, \varrho)$,

$$
\forall l \in \mathbb{N}^{6}, \quad\left\|\nabla_{z, \bar{z}, \zeta, C \zeta, f, \varrho}^{l} H_{\nu \mu}\right\|_{H_{x}^{K, S}\left(\mathbb{R}^{3}, \mathbb{C}^{2}\right)} \leq C_{l} ;
$$

(c) for $F_{j}(x, z, f, g, \varrho)$,

$$
\forall l \in \mathbb{N}^{6}, \quad\left\|\nabla_{z, \bar{z}, \zeta, C \zeta, f, \varrho}^{l} F_{j}\right\|_{H_{x}^{K, S}\left(\mathbb{R}^{3}, B\left(\left(\mathbb{C}^{2}\right)^{\otimes j}, \mathbb{C}\right)\right)} \leq C_{l} ;
$$

(d) we have $\widehat{\mathcal{R}}_{2}^{(1)}(z, f, \varrho) \in C^{\infty}\left(\mathcal{U}^{-K^{\prime},-S^{\prime}} \times \mathbb{R}, \mathbb{R}\right)$ with

$$
\left|\widehat{\mathcal{R}}_{2}^{(1)}(z, f, \varrho)\right| \leq C\left(|z|+|\varrho|+\|f\|_{H^{-K^{\prime},-S^{\prime}}}\right)\|f\|_{H^{-K^{\prime},-S^{\prime}}}^{2} .
$$

Proof. The following proof is a continuation of proof of Lemma 7.11. We thus consider $H=K \circ \mathcal{G}_{1}$ as a function of $(\varrho, U)$. By $\mathcal{G}_{1}\left(0, \Phi_{\omega_{0}}\right)=\mathcal{F}_{1}\left(\Phi_{\omega_{0}}\right)=\Phi_{\omega_{0}}, K^{\prime}\left(\Phi_{\omega_{0}}\right)=0$ and $\left\|\mathcal{F}_{1}(U)-U\right\|_{\mathcal{P}^{K, S}} \lesssim\|R\|_{L^{2}}^{2}$ we conclude $H^{\prime}\left(\Phi_{\omega_{0}}\right)=0$ and $H^{\prime \prime}\left(\Phi_{\omega_{0}}\right)=K^{\prime \prime}\left(\Phi_{\omega_{0}}\right)$. In particular, this yields the formula for $H_{2}^{(1)}+\widetilde{\mathcal{R}^{(1)}}$ for $\varrho=\|f\|_{2}^{2}=0$.

The other terms are obtained by substituting in $K_{P}$ of (8.5) the formula (7.34). The term $\psi(\varrho)$ arises from $d\left(\omega \circ \mathcal{G}_{1}\right)-\omega \circ \mathcal{G}_{1}\left\|u_{0}\right\|_{2}^{2}$. There are no monomials $\|f\|_{2}^{j} z^{\mu} \bar{z}^{\nu}\langle H, f\rangle^{i}$ with $|\mu+\nu|+i=1$, due to (7.35) (applied for $\omega=\omega_{0}$ ). By $\left\langle\mathrm{i} \beta \alpha_{2} \Sigma_{1} \Sigma_{3} f, f\right\rangle=\|f\|_{2}^{2}$, we have $\left\langle\mathrm{i} \beta \alpha_{2} \Sigma_{1} \Sigma_{3} \mathcal{H}_{\omega_{0}+\delta \omega} f, f\right\rangle=$ $\left\langle\mathrm{i} \beta \alpha_{2} \Sigma_{1} \Sigma_{3} \mathcal{H}_{\omega_{0}} f, f\right\rangle+\frac{\|f\|^{2} \varrho}{2}+\widetilde{F}_{2}$ where $\widetilde{F}_{2}$ can be absorbed in $j=2$ in $\widetilde{\mathcal{R}^{(2)}}$ and $\frac{\|f\|^{2} \varrho}{2}$ can be absorbed in $\psi$ when restricted to $\varrho=\|f\|_{2}^{2}$.

Notice that $\widehat{\mathcal{R}}_{2}^{(1)}$ is a remainder term obtained from terms in $\mathcal{E}$ of Lemme [7.11, 


\section{BIRKHOFF NORMAL FORMS}

9.1. Normal form. Here again and in the following sections, we use the notation $\lambda_{j}^{0}=\lambda_{j}\left(\omega_{0}\right)$. Set $\mathcal{H}:=\mathcal{H}_{\omega_{0}} P_{c}\left(\mathcal{H}_{\omega_{0}}\right)$.

Definition 9.1. A function $Z(z, f)$ is in normal form if it is of the form $Z=Z_{0}+Z_{1}$ where we have finite sums of the following types:

$$
Z_{1}=\sum_{\left|\lambda^{0} \cdot(\nu-\mu)\right|>m-\omega_{0}} z^{\mu} \bar{z}^{\nu}\left\langle\mathrm{i} \beta \alpha_{2} \Sigma_{1} \Sigma_{3} G_{\mu \nu}\left(\|f\|_{2}^{2}\right), f\right\rangle
$$

with $H_{\mu \nu}(x, \varrho) \in C^{\infty}\left(\mathbb{R}_{\varrho}, H_{x}^{K, S}\right)$ for all $K, S$;

$$
Z_{0}=\sum_{\lambda^{0} \cdot(\mu-\nu)=0} a_{\mu, \nu}\left(\|f\|_{2}^{2}\right) z^{\mu} \bar{z}^{\nu}
$$

and $a_{\mu, \nu}(\varrho) \in C^{\infty}\left(\mathbb{R}_{\varrho}, \mathbb{C}\right)$. We will always assume the symmetries (8.10).

We consider the coefficients of the type of (8.7) (below it will be those of the $H_{2}^{(r)}$ in Theorem 9.5) and thus let, for $\delta_{j}=\left(\delta_{1 j}, \ldots, \delta_{n j}\right)$,

$$
\lambda_{j}=\lambda_{j}\left(\|f\|_{2}^{2}\right)=\lambda_{j}^{0}+k_{\delta_{j} \delta_{j}}\left(\|f\|_{2}^{2}\right), \quad \lambda=\left(\lambda_{1}, \cdots, \lambda_{m}\right) .
$$

Let

$$
D_{2}=\sum_{j=1}^{n} \varepsilon_{j} \lambda_{j}\left(\|f\|_{2}^{2}\right)\left|z^{j}\right|+\frac{1}{2}\left\langle\mathrm{i} \beta \alpha_{2} \Sigma_{1} \Sigma_{3} \mathcal{H}_{\omega_{0}} f, f\right\rangle .
$$

We have $\left(\lambda_{j}^{\prime}(\varrho)\right.$ is the derivative in $\left.\varrho\right)$ for $F$ a scalar valued function that, summing on repeated indexes,

$$
\begin{aligned}
& \left\{D_{2}, F\right\}:=d D_{2}\left(X_{F}\right)=\partial_{j} D_{2}\left(X_{F}\right)_{j}+\partial_{\bar{j}} D_{2}\left(X_{F}\right)_{\bar{j}}+\left\langle\nabla_{f} D_{2},\left(X_{F}\right)_{f}\right\rangle \\
& =-\mathrm{i} \partial_{j} D_{2} \partial_{j} F+\mathrm{i} \partial_{j} D_{2} \partial_{j} F-\left\langle\nabla_{f} D_{2}, \beta \alpha_{2} \Sigma_{3} \Sigma_{1} \nabla_{f} F\right\rangle= \\
& \mathrm{i} \lambda_{j} z_{j} \partial_{j} F-\mathrm{i} \lambda_{j} \bar{z}_{j} \partial_{\bar{j}} F+\mathrm{i}\left\langle\mathcal{H} f, \nabla_{f} F\right\rangle+2 \mathrm{i} \lambda_{j}^{\prime}\left(\|f\|_{2}^{2}\right)\left|z_{j}\right|^{2}\left\langle f, \Sigma_{3} \nabla_{f} F\right\rangle .
\end{aligned}
$$

In particular, we have, for $G=G(x)$, (we use $\Sigma_{1} \mathrm{i} \Sigma_{2}=\Sigma_{3}$ )

$$
\begin{aligned}
& \left\{D_{2}, z^{\mu} \bar{z}^{\nu}\right\}=\mathrm{i} \lambda \cdot(\mu-\nu) z^{\mu} \bar{z}^{\nu}, \\
& \left\{D_{2},\left\langle\mathrm{i} \beta \alpha_{2} \Sigma_{1} \Sigma_{3} G, f\right\rangle\right\}=\mathrm{i}\left\langle\mathcal{H} f, \mathrm{i} \beta \alpha_{2} \Sigma_{1} \Sigma_{3} G\right\rangle-2 \mathrm{i} \sum_{j=1}^{n} \lambda_{j}^{\prime}\left|z_{j}\right|^{2}\left\langle\mathrm{i} \beta \alpha_{2} \Sigma_{1} f, G\right\rangle \\
& =-\mathrm{i}\left\langle f, \mathrm{i} \beta \alpha_{2} \Sigma_{1} \Sigma_{3} \mathcal{H} G\right\rangle-2 \mathrm{i} \sum_{j=1}^{n} \lambda_{j}^{\prime}\left|z_{j}\right|^{2}\left\langle\mathrm{i} \beta \alpha_{2} \Sigma_{1} f, G\right\rangle, \\
& \left\{D_{2}, \frac{1}{2}\|f\|_{2}^{2}\right\}=\left\{D_{2}, \frac{1}{2}\left\langle f, \mathrm{i} \beta \alpha_{2} \Sigma_{1} f\right\rangle\right\}=-\mathrm{i}\left\langle\mathcal{H} f, \mathrm{i} \beta \alpha_{2} \Sigma_{1} f\right\rangle=-\mathrm{i}\left\langle V_{\omega_{0}} f, \mathrm{i} \beta \alpha_{2} \Sigma_{1} f\right\rangle .
\end{aligned}
$$

In the sequel we will prove that $\|f\|_{2}$ is small.

Remark 9.2. We will consider only $|\mu+\nu| \leq 2 N+3$. Then, $\lambda^{0} \cdot(\mu-\nu) \neq 0$ implies $\left|\lambda^{0} \cdot(\mu-\nu)\right| \geq c>0$ for some fixed $c$, and so we can assume also $|\lambda \cdot(\mu-\nu)| \geq c / 2$. Similarly $\left|\lambda^{0} \cdot(\mu-\nu)\right|<m-\omega_{0}$ (resp. $\left.\left|\lambda^{0} \cdot(\mu-\nu)\right|>m-\omega_{0}\right)$ will be assumed equivalent to $|\lambda \cdot(\mu-\nu)|<m-\omega_{0}\left(\operatorname{resp} .|\lambda \cdot(\mu-\nu)|>m-\omega_{0}\right)$.

Lemma 9.3 (Homological equation). Consider

$$
K=\sum_{|\mu+\nu|=M_{0}+1} k_{\mu \nu}\left(\|f\|_{2}^{2}\right) z^{\mu} \bar{z}^{\nu}+\sum_{|\mu+\nu|=M_{0}} z^{\mu} \bar{z}^{\nu}\left\langle\mathrm{i} \beta \alpha_{2} \Sigma_{1} \Sigma_{3} K_{\mu \nu}\left(\|f\|_{2}^{2}\right), f\right\rangle .
$$

Suppose that all the terms in (9.7) are not in normal form and that the symmetries (8.10) hold. Consider

$$
\begin{aligned}
& \chi=\sum_{|\mu+\nu|=M_{0}+1} \frac{k_{\mu \nu}\left(\|f\|_{2}^{2}\right)}{\mathrm{i} \lambda \cdot(\mu-\nu)} z^{\mu} \bar{z}^{\nu} \\
& +\sum_{|\mu+\nu|=M_{0}} z^{\mu} \bar{z}^{\nu}\left\langle\mathrm{i} \beta \alpha_{2} \Sigma_{1} \Sigma_{3} \frac{1}{\mathrm{i}(\lambda \cdot(\mu-\nu)-\mathcal{H})} K_{\mu \nu}\left(\|f\|_{2}^{2}\right), f\right\rangle .
\end{aligned}
$$


Then we have $\left\{D_{2}, \chi\right\}=K+L$ with, summing on repeated indexes,

$$
\begin{aligned}
& L=-2 \frac{k_{\mu \nu}^{\prime}}{(\mu-\nu) \cdot \lambda} z^{\mu} \bar{z}^{\nu}\left\langle V_{\omega_{0}} f, \mathrm{i} \beta \alpha_{2} \Sigma_{1} f\right\rangle \\
& -2 \lambda_{j}^{\prime} z^{\mu} \bar{z}^{\nu}\left|z_{j}\right|^{2}\left\langle\mathrm{i} \beta \alpha_{2} \Sigma_{1} f, \frac{1}{(\mu-\nu) \cdot \lambda-\mathcal{H}} K_{\mu \nu}\right\rangle \\
& +2 \lambda^{\prime} \cdot(\mu-\nu) z^{\mu} \bar{z}^{\nu}\left|z_{j}\right|^{2}\left\langle f, \mathrm{i} \beta \alpha_{2} \frac{1}{((\mu-\nu) \cdot \lambda-\mathcal{H})^{2}} K_{\mu \nu}\right\rangle\left\langle V_{\omega_{0}} f, i \beta \alpha_{2} \Sigma_{1} f\right\rangle \\
& -2 z^{\mu} \bar{z}^{\nu}\left\langle f, \Sigma_{3} \Sigma_{1} \frac{1}{(\mu-\nu) \cdot \lambda-\mathcal{H}} K_{\mu \nu}^{\prime}\right\rangle\left\langle V_{\omega_{0}} f, i \beta \alpha_{2} \Sigma_{1} f\right\rangle .
\end{aligned}
$$

If the coefficients in (9.7) satisfy (8.10), the same is true for the coefficients in (9.8).

Proof. The proof follows by the tables (9.6), by the product rule for the derivative and by the symmetry properties of $\mathcal{H}$.

9.2. Canonical transformations. First we consider functions

$$
\chi=\sum_{|\mu+\nu|=M_{0}+1} b_{\mu \nu}\left(\|f\|_{2}^{2}\right) z^{\mu} \bar{z}^{\nu}+\sum_{|\mu+\nu|=M_{0}} z^{\mu} \bar{z}^{\nu}\left\langle\mathrm{i} \beta \alpha_{2} \Sigma_{1} \Sigma_{3} B_{\mu \nu}\left(\|f\|_{2}^{2}\right), f\right\rangle
$$

where $b_{\mu \nu}(\varrho) \in C^{\infty}\left(\mathbb{R}_{\varrho}, \mathbb{C}\right)$ and $B_{\mu \nu}(x, \varrho) \in C^{\infty}\left(\mathbb{R}, P_{c}\left(\omega_{0}\right) H_{x}^{k, s}\left(\mathbb{R}^{3}, \mathbb{C}^{8}\right)\right)$ for all $k$ and $s$. Assume

$$
b_{\mu \nu}=\left(b_{\nu \mu}\right)^{*} \text { and } \mathrm{i} \beta \alpha_{2} \Sigma_{1} B_{\mu \nu}=-\left(B_{\nu \mu}\right)^{*} \text { for all indexes. }
$$

The canonical transformations used in the proof of Theorem 9.5 are compositions of the Lie transforms $\phi:=\left.\phi^{\tau}\right|_{\tau=1}$, with $\phi^{\tau}$ the flow of the Hamiltonian vector field $X_{\chi}$ (with respect to $\Omega_{0}$ and only in $(z, f)$ ). Let for $K>0$ and $S>0$ fixed and large

$$
\|\chi\|=\sum\left|b_{\mu \nu}\left(\|f\|_{2}^{2}\right)\right|+\sum\left\|B_{\mu \nu}\left(\|f\|_{2}^{2}\right)\right\|_{H^{K, S}} .
$$

Then, the following lemma can be proved like Lemma 9.2 [26].

Lemma 9.4. Consider the $\chi$ in (9.9) and its Lie transform $\phi$. Set $\left(z^{\prime}, f^{\prime}\right)=\phi(z, f)$. Then there are $\mathcal{G}(z, f, \varrho), \Gamma(z, f, \varrho), \Gamma_{0}(z, f, \rho)$ and $\Gamma_{1}(z, f, \rho)$ with the following properties.

(1) $\Gamma \in C^{\infty}\left(\mathcal{U}^{-K^{\prime},-S^{\prime}}, \mathbb{C}^{n}\right), \Gamma_{0}, \Gamma_{1} \in C^{\infty}\left(\mathcal{U}^{-K^{\prime},-S^{\prime}}, \mathbb{R}\right)$, with $\mathcal{U}^{-K^{\prime},-S^{\prime}} \subset \mathbb{C}^{n} \times H_{c}^{-K^{\prime},-S^{\prime}}\left(\omega_{0}\right) \times \mathbb{R}$ an appropriately small neighborhood of the origin.

(2) $\mathcal{G} \in C^{\infty}\left(\mathcal{U}^{-K^{\prime},-S^{\prime}}, H_{c}^{K, S}\left(\omega_{0}\right)\right)$ for any $K, S$.

(3) The transformation $\phi$ is of the following form:

$$
\begin{gathered}
z^{\prime}=z+\Gamma\left(z, f,\|f\|_{2}^{2}\right), \\
f^{\prime}=e^{\mathrm{i} \Gamma_{0}\left(z, f,\|f\|_{2}^{2}\right) P_{c}\left(\omega_{0}\right) \Sigma_{3}} f+\mathcal{G}\left(z, f,\|f\|_{2}^{2}\right) .
\end{gathered}
$$

(4) There are constants $c_{K^{\prime}, S^{\prime}}$ and $c_{K, S, K^{\prime}, S^{\prime}}$ such that

(5) We have

$$
\begin{aligned}
\left|\Gamma\left(z, f,\|f\|_{2}^{2}\right)\right| & \leq c_{K^{\prime}, S^{\prime}}(\|\chi\|+(\overline{9.18}))|z|^{M_{0}-1}\left(|z|+\|f\|_{H^{-K^{\prime},-S^{\prime}}}\right), \\
\left\|\mathcal{G}\left(z, f,\|f\|_{2}^{2}\right)\right\|_{H^{K, S}} & \leq c_{K, S, K^{\prime}, S^{\prime}}(\|\chi\|+(9.18))|z|^{M_{0}}, \\
\left|\Gamma_{0}\left(z, f,\|f\|_{2}^{2}\right)\right| & \leq c_{K^{\prime}, S^{\prime}}|z|^{M_{0}-1}\left(|z|+\|f\|_{H^{-K^{\prime},-S^{\prime}}}\right)^{2} .
\end{aligned}
$$

$$
\begin{gathered}
\left\|f^{\prime}\right\|_{2}^{2}=\|f\|_{2}^{2}+\Gamma_{1}\left(z, f,\|f\|_{2}^{2}\right), \\
\left|\Gamma_{1}\left(z, f,\|f\|_{2}^{2}\right)\right| \leq C|z|^{M_{0}-1}\left(|z|+\|f\|_{H^{-K^{\prime},-S^{\prime}}}\right)^{2} .
\end{gathered}
$$

(6) We have

$$
e^{\mathrm{i} \Gamma_{0} P_{c}\left(\omega_{0}\right) \Sigma_{3}}=e^{\mathrm{i} \Gamma_{0} \Sigma_{3}}+T\left(\Gamma_{0}\right)
$$

where $T(r) \in C^{\infty}\left(\mathbb{R}, B\left(H^{-K^{\prime},-S^{\prime}}, H^{K, S}\right)\right)$ for all $\left(K, S, K^{\prime}, S^{\prime}\right)$, with norm

$$
\|T(r)\|_{B\left(H^{\left.-K^{\prime},-S^{\prime}, H^{K, S}\right)}\right.} \leq C\left(K, S, K^{\prime}, S^{\prime}\right)|r| .
$$

More specifically, the range of $T(r)$ is a subspace of $\mathbf{X}_{d}(\mathcal{H})+\mathbf{X}_{d}\left(\mathcal{H}^{*}\right)$.

The crux of this section is the following result. 
Theorem 9.5. For any integer $r \geq 2$ there are a neighborhood $\mathcal{U}^{1,0}$ of $\{(0,0)\}$ in $\widetilde{\mathcal{P}}^{1,0}$, see (7.26), and a smooth canonical transformation $\mathcal{T}_{r}: \mathcal{U}^{1,0} \rightarrow \widetilde{\mathcal{P}}^{1,0}$ s.t.

$$
H^{(r)}:=H \circ \mathcal{T}_{r}=d\left(\omega_{0}\right)-\omega_{0}\left\|u_{0}\right\|_{2}^{2}+\psi\left(\|f\|_{2}^{2}\right)+H_{2}^{(r)}+Z^{(r)}+\mathcal{R}^{(r)} .
$$

where:

(i) $H_{2}^{(r)}=H_{2}^{(2)}$ for $r \geq 2$, is of the form (8.7) where $k_{\mu \nu}^{(r)}\left(\|f\|_{2}\right)$ satisfy (8.9)- (8.10);

(ii) $Z^{(r)}$ is in normal form, in the sense of Definition 9.1 above, with monomials of degree $\leq r$ whose coefficients satisfy (8.10);

(iii) the transformation $\mathcal{T}_{r}$ is of the form (9.12) - (9.13) and satisfies (9.14) - (9.16) for $M_{0}=1$;

(iv) we have $\mathcal{R}^{(r)}=\sum_{d=0}^{6} \mathcal{R}_{d}^{(r)}$ and for all $\left(K, S, K^{\prime}, S^{\prime}\right)$ positives there is a neighbourhood $\mathcal{U}^{-K^{\prime},-S^{\prime}}$ of $\{(0,0)\}$ in $\widetilde{\mathcal{P}}^{-K^{\prime},-S^{\prime}}$ such that (iv.0)

$$
\mathcal{R}_{0}^{(r)}=\sum_{|\mu+\nu|=r+1} z^{\mu} \bar{z}^{\nu} \int_{\mathbb{R}^{3}} k_{\mu \nu}^{(r)}\left(x, z, f, f(x),\|f\|_{2}^{2}\right) d x
$$

and for $k_{\mu \nu}^{(r)}(z, f, \eta, \varrho)$ with ${ }^{t} \eta=(\zeta, C \zeta), \zeta \in \mathbb{C}^{4}$ we have for $(z, f) \in \mathcal{U}^{-K^{\prime},-S^{\prime}}$ and $|\varrho| \leq 1$

(iv.1)

$$
\left\|\nabla_{z, \bar{z}, \zeta, C \zeta, f, \varrho}^{l} \varrho_{\mu \nu}^{(r)}(\cdot, z, f, \eta, \varrho)\right\|_{H^{K, S}\left(\mathbb{R}^{3}, \mathbb{C}\right)} \leq C_{l} \text { for all } l
$$

$$
\begin{gathered}
\mathcal{R}_{1}^{(r)}=\sum_{|\mu+\nu|=r} z^{\mu} \bar{z}^{\nu} \int_{\mathbb{R}^{3}}\left[\mathrm{i} \beta \alpha_{2} \Sigma_{1} \Sigma_{3} H_{\mu \nu}^{(r)}\left(x, z, f, f(x),\|f\|_{2}^{2}\right)\right]^{T} f(x) d x \\
\text { with }\left\|\nabla_{z, \bar{z}, \zeta, C \zeta, f, \varrho}^{l} H_{\nu \mu}^{(r)}(\cdot, z, f, \eta, \varrho)\right\|_{H^{K, S}\left(\mathbb{R}^{3}, \mathbb{C}^{8}\right)} \leq C_{l} \text { for all } l ;
\end{gathered}
$$

(iv.2-5) for $2 \leq d \leq 5$,

$$
\mathcal{R}_{d}^{(r)}=\int_{\mathbb{R}^{3}} F_{d}^{(r)}\left(x, z, f, f(x),\|f\|_{2}^{2}\right) f^{\otimes d}(x) d x+\widehat{\mathcal{R}}_{d}^{(r)},
$$

with for any $l$

$$
\begin{gathered}
\left\|\nabla_{z, \bar{z}, \zeta, C \zeta, f, \varrho}^{l} F_{d}^{(r)}(\cdot, z, f, \eta, \varrho)\right\|_{H^{K, S}\left(\mathbb{R}^{3}, B\left(\left(\mathbb{C}^{8}\right) \otimes d, \mathbb{C}\right)\right.} \leq C_{l}, \\
\text { with } F_{2}^{(r)}(x, 0,0,0,0)=0 \text { and with } \widetilde{\mathcal{R}}_{d}^{(r)}\left(z, f,\|f\|_{2}^{2}\right) \text { s.t. } \\
\widehat{\mathcal{R}}_{d}^{(r)}(z, f, \varrho) \in C^{\infty}\left(\mathcal{U}^{-K^{\prime},-S^{\prime}} \times \mathbb{R}, \mathbb{R}\right), \\
\left|\widehat{\mathcal{R}}_{d}^{(r)}(z, f, \varrho)\right| \leq C\|f\|_{H^{-K^{\prime},-S^{\prime}}}^{d}, \\
\left|\widehat{\mathcal{R}}_{2}^{(r)}(z, f, \varrho)\right| \leq C\left(|z|+|\varrho|+\|f\|_{\left.H^{-K^{\prime},-S^{\prime}}\right)\|f\|_{H^{-K^{\prime},-S^{\prime}}}^{2}}\right. \\
\text { (iv.6) } \mathcal{R}_{6}^{(r)}=\int_{\mathbb{R}^{3}}
\end{gathered}
$$

The proof of Theorem 9.5] is the same of Theorem 9.1 in [26] and we skip it. The ingredients needed in the proof (in particular the notion of normal form) are described above.

\section{NON LINEAR DYNAMICS}

10.1. Dispersion. $\lambda$ We apply Theorem 9.5 for $r=2 N_{1}+1$ (recall $N_{j} \lambda_{j}<m-\omega_{0}<\left(N_{j}+1\right) \lambda_{j}$ ). In the rest of the article we work with the Hamiltonian $H^{(r)}$. We will drop the upper index. So we will set $H=H^{(r)}, H_{2}=H_{2}^{(r)}, \lambda_{j}=\lambda_{j}^{(r)}, \lambda=\lambda^{(r)}, Z_{a}=Z_{a}^{(r)}$ for $a=0,1$ and $\mathcal{R}=\mathcal{R}^{(r)}$. In particular we will denote by $H_{\mu \nu}$ the coefficients $G_{\mu \nu}^{(r)}$ of $Z_{1}^{(r)}$. We will show: 
Theorem 10.1. Fix $p_{0}>2$ and $\tau_{0}>1$. Let $\frac{2}{p}=\frac{3}{2}\left(1-\frac{2}{q}\right)$ and $\alpha(q)=\frac{2}{p}$, i.e. $\left(1+\frac{\theta}{2}\right)\left(1-\frac{2}{q}\right)=\frac{2}{p}$ with $\theta=1$ in Theorem 5.6. Consider $k_{0} \geq 4, k_{0} \in \mathbb{Z}, \epsilon \in\left(0, \varepsilon_{0}\right)$ and $\varepsilon_{0}>0$ as in Theorem 1.3). Then there is a fixed $C>0$ such that for $\varepsilon_{0}>0$ sufficiently small and for $p \geq p_{0}$ we have the following inequalities:

$$
\begin{gathered}
\|f\|_{L_{t}^{p}\left([0, \infty), B_{q, 2}^{k_{0}-\frac{2}{p}}\right)} \leq C \epsilon ; \\
\|f\|_{L_{t}^{2}\left([0, \infty), H_{x}^{k_{0},-\tau_{0}}\right)} \leq C \epsilon \\
\|f\|_{L_{t}^{2}\left([0, \infty), L_{x}^{\infty}\right)} \leq C \epsilon \\
\left\|z^{\mu}\right\|_{L_{t}^{2}([0, \infty))} \leq C \epsilon \text { for all multi indexes } \mu \text { with } \lambda \cdot \mu>m-\omega_{0} \\
\left\|z_{j}\right\|_{W_{t}^{1, \infty}([0, \infty))} \leq C \epsilon \text { for all } j \in\{1, \ldots, n\} .
\end{gathered}
$$

Due to time reversibility, it is easy to conclude that (10.1)-10.5) are true over the whole real line.

The proof of Theorem 10.1 involves a standard continuation argument following [51, End of proof of Theorem II.2.1]. We assume

$$
\begin{gathered}
\|f\|_{L_{t}^{p}\left([0, T], B_{q, 2}^{k_{0}-\frac{2}{p}}\right)}+\|f\|_{L_{t}^{2}\left([0, T], H_{x}^{\left.k_{0},-\tau_{0}\right)}\right.}+\|f\|_{L_{t}^{2}\left([0, T], L_{x}^{\infty}\right)} \leq C_{1} \epsilon \\
\left\|z^{\mu}\right\|_{L_{t}^{2}([0, T])} \leq C_{2} \epsilon \text { for all multi indexes } \mu \text { with } \omega \cdot \mu>m-\omega_{0} \\
\left\|z_{j}\right\|_{W_{t}^{1, \infty}([0, T])} \leq C_{3} \epsilon \text { for all } j \in\{1, \ldots, n\}
\end{gathered}
$$

for fixed sufficiently large constants $C_{1}-C_{3}$. Notice that there is an $\varepsilon_{1}>0$ such that this assumption is true for all $|z(0)|+\|f(0)\|_{H^{k_{0}}}<\varepsilon_{1}$ if say $T \in(0,1]$. We then prove that there exists a fixed $\varepsilon_{0} \in\left(0, \varepsilon_{1}\right)$, with $\varepsilon_{0}=\varepsilon_{0}\left(C_{1}, C_{2}, C_{3}\right)$, such that for $\epsilon \in\left(0, \varepsilon_{0}\right)$, (10.6) -(10.8) imply the same estimate but with $C_{1}-C_{3}$ replaced by $C_{1} / 2-C_{3} / 2$. This implies that the set of $T$ such that (10.6)-(10.8) is open in $\mathbb{R}^{+}$. Since it is also closed, it is all $\mathbb{R}^{+}$. Then (10.6)-(10.8) hold with $[0, T]$ replaced by $[0, \infty)$ for all $|z(0)|+\|f(0)\|_{H^{k_{0}}}<$ $\epsilon<\varepsilon_{0}$.

The proof of Theorem 10.1 consists in three main steps.

(i) Estimate $f$ in terms of $z$.

(ii) Substitute the variable $f$ with a new "smaller" variable $g$ and find smoothing estimates for $g$.

(iii) Reduce the system for $z$ to a closed system involving only the $z$ variables, by insulating the part of $f$ which interacts with $z$, and by decoupling the rest (this reminder is $g$ ). Then clarify the nonlinear Fermi golden rule.

Step (i). Using the Proposition 10.2 below, we will choose $C_{1}>2 K_{1}\left(C_{2}\right)$. This tells us that if we get upper bounds on $C_{2}$ and $C_{3}$, and this is done in Sect. 11, then we will have proved Theorem 10.1.

Proposition 10.2. Assume (10.6)-(10.8). Then there exist constants $C=C\left(C_{1}, C_{2}, C_{3}\right), K_{1}\left(C_{2}\right)$, such that, if $C\left(C_{1}, C_{2}, C_{3}\right) \epsilon$ is sufficiently small, then we have

$$
\|f\|_{L_{t}^{p}\left([0, T], B_{q, 2}^{\left.k_{0}-\frac{2}{p}\right)}\right.}+\|f\|_{L_{t}^{2}\left([0, T], H_{x}^{k_{0},-\tau_{0}}\right)}+\|f\|_{L_{t}^{2}\left([0, T], L_{x}^{\infty}\right)} \leq K_{1}\left(C_{2}\right) \epsilon .
$$

Proof. Consider $Z_{1}$ of the form (9.1). Set:

$$
H_{\mu \nu}^{0}=H_{\mu \nu}\left(\|f\|_{2}^{2}\right) \text { for }\|f\|_{2}^{2}=0 ; \lambda_{j}^{0}=\lambda_{j}\left(\omega_{0}\right) .
$$

Then we have (with finite sums)

$$
\begin{aligned}
& \text { if } \dot{f}-\mathcal{H} f-2\left(\partial_{\|f\|_{2}^{2}} H\right) P_{c}\left(\omega_{0}\right) \Sigma_{3} f=\sum_{\substack{\left|\lambda^{0} \cdot(\nu-\mu)\right|>m-\omega_{0},|\mu+\nu| \leq 2 N_{1}+1}} z^{\mu} \bar{z}^{\nu} H_{\mu \nu}^{0} \\
& +\sum_{\substack{\left|\lambda^{0} \cdot(\nu-\mu)\right|>m-\omega_{0},|\mu+\nu| \leq 2 N_{1}+1}} z^{\mu} \bar{z}^{\nu}\left(H_{\mu \nu}-H_{\mu \nu}^{0}\right)+\mathrm{i} \beta \alpha_{2} \Sigma_{3} \Sigma_{1} \nabla_{f} \mathcal{R}-2\left(\partial_{\|f\|_{2}^{2}} \mathcal{R}\right) P_{c}\left(\omega_{0}\right) \Sigma_{3} f .
\end{aligned}
$$

In order to obtain bounds on $f$, we need bounds on the right hand term of the equation especially the last two terms. They are provided by the following lemma.

Lemma 10.3. Assume (10.6)-(10.8) and consider a fixed $\tau_{0}>1$. Then there is a constant $C=$ $C\left(C_{1}, C_{2}, C_{3}\right)$ independent of $\epsilon$ such that the following is true: we have

$$
\beta \alpha_{2} \Sigma_{3} \Sigma_{1} \nabla_{f} \mathcal{R}-2\left(\partial_{\|f\|_{2}^{2}} \mathcal{R}\right) P_{c}\left(\omega_{0}\right) \Sigma_{3} f=R_{1}+R_{2}
$$


with

$$
\begin{aligned}
& \left\|R_{1}\right\|_{H_{x}^{k_{0}}} \leq C\left(C_{1}, C_{2}, C_{3}\right)\left(|z|^{2 N_{1}+2}+\|f\|_{L^{\infty}}^{2}\|f\|_{H_{x}^{k_{0}}}\right) \\
& \left\|R_{2}\right\|_{H_{x}^{k_{0}}, \tau_{0}} \leq C\left(C_{1}, C_{2}, C_{3}\right)\left(|z|+\|f\|_{L_{x}^{2}}^{2}+\|f\|_{H_{x}^{k_{0},-\tau_{0}}}\right)\|f\|_{H_{x}^{k_{0},-\tau_{0}}} .
\end{aligned}
$$

In particular we have for some other fixed constant $C=C\left(C_{1}, C_{2}, C_{3}\right)$,

$$
\left\|R_{1}\right\|_{L_{t}^{1}\left([0, T], H_{x}^{k_{0}}\right)}+\left\|R_{2}\right\|_{L_{t}^{2}\left([0, T], H_{x}^{k_{0}, \tau_{0}}\right)} \leq C\left(C_{1}, C_{2}, C_{3}\right) \epsilon^{2} .
$$

Proof. (10.13) is a consequence of (10.12) and (10.6)-(10.8). We focus on (10.12). For $d \leq 1$ and arbitrary fixed $(S, K)$ we have $\nabla_{f} \mathcal{R}_{d} \in H^{S, K}$. By (iv0-iv1) Theorem 9.5

$$
\left\|\nabla_{f} \mathcal{R}_{0}\right\|_{H^{S, K}}+\left\|\nabla_{f} \mathcal{R}_{1}\right\|_{H^{S, K}} \leq C|z|^{2 N_{1}+2} .
$$

These terms can be absorbed in $R_{1}$. For $2 \leq d \leq 5$ we have

$$
\Sigma_{3} \Sigma_{1} \nabla_{f} \widehat{\mathcal{R}}_{d}-2\left(\partial_{\|f\|_{2}^{2}} \widehat{\mathcal{R}}_{d}\right) P_{c}\left(\omega_{0}\right) \Sigma_{3} f=\Sigma_{3} \Sigma_{1} \nabla_{f} \widehat{\mathcal{R}}_{d}(z, f, \rho),
$$

computed at $\rho=\|f\|_{2}^{2}$. By (9.24) we obtain

$$
\begin{aligned}
& \left\|\nabla_{f} \widehat{\mathcal{R}}_{d}(z, f, \rho)\right\|_{H^{K^{\prime}, S^{\prime}}} \leq C\|f\|_{H^{-K^{\prime},-S^{\prime}}}^{d-1} \text { for } 3 \leq d \leq 5 \text { and } \\
& \left\|\nabla_{f} \widehat{\mathcal{R}}_{2}(z, f, \rho)\right\|_{H^{K^{\prime}, S^{\prime}}} \leq C\|f\|_{H^{-K^{\prime},-S^{\prime}}}^{2}+C|z|\|f\|_{H^{-K^{\prime},-S^{\prime}}}
\end{aligned}
$$

Since $K^{\prime}$ and $S^{\prime}$ are arbitrarily large, we have $\|f\|_{H^{-K^{\prime},-S^{\prime}}} \leq\|f\|_{H^{k_{0},-\tau_{0}}}$. So these terms can be absorbed in $R_{2}$. Other terms are treated as in [2, Lemma 7.5] : For $d=2,3,4,5$ we have schematically

$$
\begin{aligned}
& F_{d}(x, z, f, f(t, \cdot), \rho) f^{\otimes(d-1)}(t, \cdot)+\partial_{w} F_{d}(x, z, f, w, \rho)_{w=f(t, \cdot)} f^{\otimes d}(t, \cdot) \\
& +\nabla_{g}\left(\int_{\mathbb{R}^{3}} F_{d}\left(x, z, g, f(t, x),\|f(t)\|_{L_{x}^{2}}^{2}\right)[f(t, x)]^{\otimes d} d x\right)_{g=f} .
\end{aligned}
$$

The first line of (10.14) has $H_{x}^{k_{0}, \tau_{0}}$ norm bounded, for some fixed sufficiently large $\mathbf{N}$, by

$$
\begin{aligned}
& \widetilde{C}\left\|\langle x\rangle^{\mathbf{N}} F_{d}(x, z, f, f(t, x), \rho)\right\|_{W_{x}^{k_{0}, \infty}}\|f\|_{H_{x}^{k_{0},-\tau_{0}}}^{d-1} \\
& +\widetilde{C}\left\|\langle x\rangle^{\mathbf{N}} \partial_{w} F_{d}(x, z, f, w, \rho)_{w=f(t, x)}\right\|_{W_{x}^{k_{0}, \infty}}\|f\|_{H_{x}^{k_{0},-\tau_{0}}}^{d} \leq C\|f\|_{H_{x}^{k_{0},-\tau_{0}}}^{d-1}+C\|f\|_{H_{x}^{k_{0},-\tau_{0}}}^{d} .
\end{aligned}
$$

When these terms are bounded by $\|f\|_{H_{x}^{k_{0},-\tau_{0}}}^{d_{1}}$ for $d_{1} \geq 2$, we can absorb them in $R_{2}$. Cases $d_{1}=1$ come from terms in the first line of (10.15) with $d=2$. By $F_{2}(x, 0,0,0,0)=0$ these are less than

$$
\left(|z|+\|f\|_{H_{x}^{-K^{\prime},-S^{\prime}}}+\|f\|_{L_{x}^{2}}^{2}\right)\|f\|_{H_{x}^{k_{0},-\tau_{0}}}
$$

and can be absorbed in $R_{2}$. Looking at the second line of (10.14) and for $\mathbf{N}$ sufficiently large, we have

$$
\begin{aligned}
& \left\|\nabla_{g}\left(\int_{\mathbb{R}^{3}} F_{d}\left(x, z, g, f(t, x),\|f(t)\|_{L_{x}^{2}}^{2}\right)[f(t, x)]^{\otimes d} d x\right)_{g=f}\right\|_{H_{x}^{k_{0}}}= \\
& \left|\sup _{\|\psi\|_{H_{x}^{-k_{0}}=1}} \int_{\mathbb{R}^{3}} D_{g} F_{d}\left(x, z, g, f(t, x),\|f(t)\|_{L_{x}^{2}}^{2}\right)_{g=f}[\psi][f(t, x)]^{\otimes d} d x\right| \\
& \leq C \sup _{\|\psi\|_{H_{x}^{-k_{0}}}=1}\left\|D_{g} F_{d}\left(x, z, g, f(t, x),\|f(t)\|_{L_{x}^{2}}^{2}\right)_{g=f}[\psi]\right\|_{L_{x}^{\infty, \mathbf{N}}}\|f\|_{H_{x}^{k_{0},-\tau_{0}}}^{d} \leq C\|f\|_{H_{x}^{k_{0},-\tau_{0}}}^{d} .
\end{aligned}
$$

So the second line of (10.14) can be absorbed in $R_{2}$. Finally we consider $\nabla_{f} \mathcal{R}_{6}=\Sigma_{1} g\left(|f(t, x)|^{2} / 2\right) f(t, x)$. Then for a fixed $C$ we have $\left\|\nabla_{f} \mathcal{R}_{6}\right\|_{H_{x}^{k_{0}}} \leq C\|f\|_{L_{x}^{\infty}}^{2}\|f\|_{H_{x}^{k_{0}}}$.

Denote by $F$ the rhs of (10.11) and set $\varphi=2 \partial_{\|f\|_{2}^{2}} H$.

Lemma 10.4. Consider i $\dot{\psi}-\mathcal{H} \psi-\varphi(t) \Sigma_{3} P_{c} \psi=F$ where $P_{c}=P_{c}\left(\omega_{0}\right)$ and $\psi=P_{c} \psi$. Let $k \in \mathbb{Z}$ with $k \geq 0$ and $\tau_{0}>1$. Then there exist $c_{0}>0$ and $C>0$ such that if $\|\varphi\|_{L_{t}^{\infty}[0, T]}<c_{0}$ then for $p \geq p_{0}>2$ and for $(p, q)$ as in Theorem 10.1 we have

$$
\|\psi\|_{L_{t}^{p}\left([0, T], B_{q, 2}^{k-\frac{2}{p}}\right) \cap L_{t}^{2}\left([0, T], H_{x}^{k,-\tau_{0}}\right)} \leq C\|\psi(0)\|_{H^{k}}+C\|F\|_{L_{t}^{1}\left([0, T], H_{x}^{k}\right)+L_{t}^{2}\left([0, T], H_{x}^{k, \tau}\right)}
$$


Proof. We apply the argument for the NLS in Lemma B.2 [44, see also Theorem 1.5 [5]. A more precise statement than Lemma B.2 44 is in 13, 23, but the proof does not seem easy to reproduce for Dirac. We fix any $\delta>0$. Let $P_{d}=P_{d}\left(\omega_{0}\right)$ and $\mathcal{H}_{0}=\mathcal{H}_{\omega_{0}, 0}$. Consider

$$
\mathrm{i} \dot{Z}-\mathcal{H} P_{c} Z+\mathrm{i} \delta P_{d} Z-\varphi \Sigma_{3} P_{c} Z=F \text {. }
$$

Then notice that for $Z(0)=\psi(0)$ the solution of (10.17) satisfies $Z(t) \equiv \psi(t)$. We rewrite (10.17) as

$$
\mathrm{i} \dot{Z}-\mathcal{H}_{0} Z-\varphi \Sigma_{3} Z=F+\left(V-\mathcal{H} P_{d}-\mathrm{i} \delta P_{d}\right) Z-\varphi \Sigma_{3} P_{d} Z \text {. }
$$

Let $\left(V-\mathcal{H} P_{d}-\mathrm{i} \delta P_{d}\right)=V_{1} V_{2}$ with $V_{2}(x)$ a smooth exponentially decaying and invertible matrix, and with $V_{1}$ bounded from $H^{k, s^{\prime}} \rightarrow H^{k, s}$ for all $k, s$ and $s^{\prime}$. For $\mathcal{U}(t)=e^{-\mathrm{i} \Sigma_{3} \int_{0}^{t} \varphi\left(t^{\prime}\right) d t^{\prime}}$ we have

$$
Z(t)=\mathcal{U}(t) e^{-\mathrm{i} \mathcal{H}_{0} t} Z(0)-\mathrm{i} \int_{0}^{t} e^{\mathrm{i} \mathcal{H}_{0}\left(t^{\prime}-t\right)} \mathcal{U}(t) \mathcal{U}^{-1}\left(t^{\prime}\right)\left[F\left(t^{\prime}\right)+V_{1} V_{2} Z\left(t^{\prime}\right)-\varphi\left(t^{\prime}\right) \Sigma_{3} P_{d} Z\left(t^{\prime}\right)\right] d t^{\prime} .
$$

$c_{0} P_{d} V_{2}^{-1}$ maps $H^{-K^{\prime},-S^{\prime}} \rightarrow H^{K, S}$ for arbitrarily fixed pairs $(K, S)$ and $\left(K^{\prime}, S^{\prime}\right)$. By picking $c_{0}$ small enough, we can assume that the related operator norms are small. By Theorems 5.4 and 5.6

$$
\begin{aligned}
& \|Z\|_{L_{t}^{p} B_{q, 2}^{k-\frac{2}{p}} \cap L_{t}^{2} H_{x}^{k,-\tau_{0}}} \leq C\|Z(0)\|_{H^{k}}+C\|F\|_{L_{t}^{1} H_{x}^{k}+L_{t}^{2} H_{x}^{k, \tau_{0}}} \\
& +\left\|V_{1}-\varphi(t) \Sigma_{3} P_{d} V_{2}^{-1}\right\|_{L_{t}^{\infty} B\left(H_{x}^{k}, H_{x}^{k, \tau_{0}}\right)}\left\|V_{2} Z(t)\right\|_{L_{t}^{2} H_{x}^{k} .}
\end{aligned}
$$

For $\widetilde{T}_{0} f(t)=V_{2} \int_{0}^{t} e^{\mathrm{i} \mathcal{H}_{0}\left(t^{\prime}-t\right)} \mathcal{U}(t) \mathcal{U}^{-1}\left(t^{\prime}\right) V_{1} f\left(t^{\prime}\right) d t^{\prime}$, by (10.18) we obtain

$$
\left(I+\mathrm{i} \widetilde{T}_{0}\right) V_{2} Z(t)=V_{2} \mathcal{U}(t) e^{-\mathrm{i} \mathcal{H}_{0} t} Z(0)-\mathrm{i} V_{2} \int_{0}^{t} e^{\mathrm{i} \mathcal{H}_{0}\left(t^{\prime}-t\right)} \mathcal{U}(t) \mathcal{U}^{-1}\left(t^{\prime}\right)\left[F\left(t^{\prime}\right)-\varphi\left(t^{\prime}\right) \Sigma_{3} P_{d} Z\left(t^{\prime}\right)\right] d t^{\prime}
$$

We then obtain (10.16) if we can show that

$$
\left\|\left(I+\mathrm{i} \widetilde{T}_{0}\right)^{-1}: L_{t}^{2}\left([0, T), H^{k}\left(\mathbb{R}^{3}\right)\right) \rightarrow L_{t}^{2}\left([0, T), H^{k}\left(\mathbb{R}^{3}\right)\right)\right\|<C_{1},
$$

for $c_{0} C_{1}$ smaller than a fixed number. It is enough to prove (10.19) with $\widetilde{T}_{0}$ replaced by

Indeed by Theorem 5.5 we have

$$
T_{0} f(t)=V_{2} \int_{0}^{t} e^{\mathrm{i} \mathcal{H}_{0}\left(t^{\prime}-t\right)} V_{1} f\left(t^{\prime}\right) d t^{\prime} .
$$

$$
\begin{aligned}
& \left\|\left(\widetilde{T}_{0}-T_{0}\right) f\right\|_{L_{t}^{2} H_{x}^{k}} \leq\left\|\int_{0}^{t}\right\| V_{2} e^{\mathrm{i} \mathcal{H}_{0}\left(t^{\prime}-t\right)}\left(e^{\mathrm{i} \Sigma_{3} \int_{t}^{t^{\prime}} \varphi\left(t^{\prime \prime}\right) d t^{\prime \prime}}-1\right) V_{1} f\left(t^{\prime}\right)\left\|_{H_{x}^{k}} d t^{\prime}\right\|_{L_{t}^{2}} \\
& \leq \widetilde{C} c_{0}^{\frac{1}{4}}\left\|\int_{0}^{t}\left\langle t^{\prime}-t\right\rangle^{-\frac{5}{4}}\right\| f\left(t^{\prime}\right)\left\|_{H_{x}^{k}} d t^{\prime}\right\|_{L_{t}^{2}} \leq C c_{0}^{\frac{1}{4}}\left\|f\left(t^{\prime}\right)\right\|_{L_{t}^{2} H_{x}^{k}} .
\end{aligned}
$$

Set

$$
T_{1} f(t)=V_{2} \int_{0}^{t} e^{\left(\mathrm{i} \mathcal{H} P_{c}+\delta P_{d}\right)\left(t^{\prime}-t\right)} V_{1} f\left(t^{\prime}\right) d t^{\prime}=V_{2} \int_{0}^{t}\left(e^{\left(\mathrm{i} \mathcal{H}\left(t^{\prime}-t\right)\right.} P_{c}+e^{-\delta\left|t^{\prime}-t\right|} P_{d}\right) V_{1} f\left(t^{\prime}\right) d t^{\prime} .
$$

By Lemma 5.9 we have $\left\|T_{1}: L_{t}^{2}\left([0, T), H^{k}\left(\mathbb{R}^{3}\right)\right) \rightarrow L_{t}^{2}\left([0, T), H^{k}\left(\mathbb{R}^{3}\right)\right)\right\|<C_{2}$ for a fixed $C_{2}$. For exactly the same reasons of [4] we have

$$
\left(I+\mathrm{i} T_{0}\right)\left(I-\mathrm{i} T_{1}\right)=\left(I-\mathrm{i} T_{1}\right)\left(I+\mathrm{i} T_{0}\right)=I .
$$

This yields (10.19) with $\widetilde{T}_{0}$ replaced by $T_{0}$ and with $C_{1}=1+C_{2}$.

Lemma 10.5. Using the notation of Lemma 10.4. but this time picking $\tau_{0}>3 / 2$, we have

$$
\|\psi\|_{L_{t}^{2}\left([0, T], L^{\infty}\right)} \leq C\|\psi(0)\|_{H^{k_{0}}}+C\|F\|_{L_{t}^{1}\left([0, T], H_{x}^{k_{0}}\right)+L_{t}^{2}\left([0, T], H_{x}^{k_{0}, \tau_{0}}\right)}
$$

Proof. We proceed as above until (10.18). We claim we have

$$
\begin{aligned}
& \|Z\|_{L_{t}^{2} L_{x}^{\infty}} \leq C\|Z(0)\|_{H^{k_{0}}}+C\|F\|_{L_{t}^{1} H_{x}^{k_{0}}+L_{t}^{2} H_{x}^{k_{0}, \tau_{0}}} \\
& +\left\|V_{1}-\varphi(t) \Sigma_{3} P_{d} V_{2}^{-1}\right\|_{L_{t}^{\infty} B\left(H_{x}^{k_{0}}, H_{x}^{k_{0}, \tau_{0}}\right)}\left\|V_{2} Z(t)\right\|_{L_{t}^{2} H_{x}^{k_{0}}} .
\end{aligned}
$$

(10.21) will yield (10.20) by the argument in Lemma 10.4. So now we prove (10.21). We have for $k>1 / 2$

$$
\left\|e^{-\mathrm{i} \mathcal{H}_{0} t} Z(0)\right\|_{L_{t}^{2} L_{x}^{\infty}} \leq C\left\|e^{-\mathrm{i} \mathcal{H}_{0} t} Z(0)\right\|_{L_{t}^{2} B_{6,2}^{k}} \leq C^{\prime}\|Z(0)\|_{H^{k+1}} \leq C^{\prime}\|Z(0)\|_{H^{k_{0}}}
$$

by Theorem 5.6. Similarly, splitting $F=F_{1}+F_{2}$, we have 


$$
\begin{aligned}
& \left\|\int_{0}^{t} e^{\mathrm{i} \mathcal{H}_{0}\left(t^{\prime}-t\right)} \mathcal{U}^{-1}\left(t^{\prime}\right) F_{1}\left(t^{\prime}\right) d t^{\prime}\right\|_{L_{t}^{2} L_{x}^{\infty}} \leq C\left\|\int_{0}^{t} e^{\mathrm{i} \mathcal{H}_{0}\left(t^{\prime}-t\right)} \mathcal{U}^{-1}\left(t^{\prime}\right) F_{1}\left(t^{\prime}\right) d t^{\prime}\right\|_{L_{t}^{2} B_{6,2}^{k}} \\
& \leq C^{\prime}\left\|F_{1}\right\|_{L_{t}^{1} H^{k+1}} \leq C^{\prime}\left\|F_{1}\right\|_{L_{t}^{1} H^{k_{0}}} .
\end{aligned}
$$

Using $B_{\infty, 2}^{k} \subset L^{\infty}$ for $k>0$, by Theorem $3.1[9]$ we have for $k_{0}>3$

$$
\begin{aligned}
& \left\|\int_{0}^{t} e^{i \mathcal{H} \mathcal{H}_{0}\left(t^{\prime}-t\right)} \mathcal{U}^{-1}\left(t^{\prime}\right) F_{2}\left(t^{\prime}\right) d t^{\prime}\right\|_{L_{t}^{2} L_{x}^{\infty}} \leq C\left\|\int_{0}^{t} \min \left\{\left|t-t^{\prime}\right|^{-\frac{1}{2}},\left|t-t^{\prime}\right|^{-\frac{3}{2}}\right\}\right\| F_{2}\left(t^{\prime}\right)\left\|_{B_{1,2}^{k_{0}}} d t^{\prime}\right\|_{L_{t}^{2}} \\
& \leq C^{\prime}\left\|F_{2}\right\|_{L_{t}^{2} B_{1,2}^{k_{0}}} \leq C^{\prime \prime}\left\|\langle x\rangle^{\tau_{0}} F_{2}\right\|_{L_{t}^{2} B_{2,2}^{k_{0}}}=C^{\prime \prime}\left\|F_{2}\right\|_{L_{t}^{2} H^{k_{0}, \tau_{0}}},
\end{aligned}
$$

where we have used $\left\|\varphi_{j} * F_{2}\right\|_{L_{x}^{1}} \leq\left\|\langle x\rangle^{-\tau_{0}}\right\|_{L_{x}^{2}}\left\|\langle x\rangle^{\tau_{0}} \varphi_{j} * F_{2}\right\|_{L_{x}^{2}} \leq C^{\prime \prime \prime}\left\|\varphi_{j} *\left(\langle\cdot\rangle^{\tau_{0}} F_{2}\right)\right\|_{L_{x}^{2}}$ for fixed $C^{\prime \prime \prime}>0$ and fixed $\tau_{0}>3 / 2$. With $F_{2}$ replaced by $\left(V_{1} V_{2}-\varphi \Sigma_{3} P_{d}\right) Z$ we get a similar estimate. This yields inequality (10.21).

Continuation of the proof of Proposition 10.1. By (10.11) we can apply to $f$ Lemmas 10.4 and 10.5 by taking $\varphi(t)=2\left(\partial_{\|f\|_{2}^{2}} H\right)$ and $F=\operatorname{rhs}(10.11)-\varphi(t)\left[\Sigma_{3}, P_{d}\right] f$. Then

$$
\|f\|_{L_{t}^{p}\left([0, T], B_{q, 2}^{k_{0}-\frac{2}{p}}\right) \cap L_{t}^{2}\left([0, T], H_{x}^{k_{0},-\tau_{0}}\right) \cap L_{t}^{2}\left([0, T], L_{x}^{\infty}\right)} \leq C\|f(0)\|_{H^{k_{0}}}+C\|F\|_{L_{t}^{1}\left([0, T], H_{x}^{k_{0}}\right)+L_{t}^{2}\left([0, T], H_{x}^{k_{0}, s}\right)} .
$$

We have

$$
\|F\|_{L_{t}^{1} H_{x}^{k_{0}}+L_{t}^{2} H_{x}^{k_{0}, \tau_{0}}} \lesssim \sum_{\lambda \cdot \mu>m-\omega_{0}}\left\|z^{\mu}\right\|_{L_{t}^{2}}^{2}+\left\|R_{1}\right\|_{L_{t}^{1} H_{x}^{k_{0}}}+\left\|R_{2}\right\|_{L_{t}^{2} H_{x}^{k_{0}, \tau_{0}}}+\epsilon\|f\|_{L_{t}^{2} H_{x}^{-k_{0},-\tau_{0}}}
$$

For $\epsilon$ small this yields Proposition 10.1 by Lemma 10.4 and by (10.7).

Lemma 10.6. Assume the conclusions of Theorem 10.1. Then there exists a fixed $C>0$ and $f_{+}^{\prime} \in H^{k_{0}}$ with $\left\|f_{+}^{\prime}\right\|_{H^{k_{0}}}<C \epsilon$ such that for for $\vartheta(t)$ the phase in the ansatz (4.1) we have

$$
\lim _{t \rightarrow+\infty}\left\|e^{\mathrm{i} \vartheta(t) \Sigma_{3}} f(t)-e^{-\mathrm{i} t D_{m}} f_{+}^{\prime}\right\|_{H^{k_{0}}}=0 .
$$

Proof. For $\psi(t)=f(t)$, for $F=\operatorname{rhs}(10.11)-\varphi(t)\left[\Sigma_{3}, P_{d}\right] f$ and for $t_{1}<t_{2}$, we have

$$
\begin{aligned}
& \left\|\mathcal{U}^{-1}\left(t_{2}\right) e^{\mathrm{i} \mathcal{H}_{0} t_{2}} f\left(t_{2}\right)-\mathcal{U}^{-1}\left(t_{1}\right) e^{\mathrm{i} \mathcal{H}_{0} t_{1}} f\left(t_{1}\right)\right\|_{H^{k_{0}}} \\
& \leq\left\|\int_{t_{1}}^{t_{2}} e^{\mathrm{i} \mathcal{H}_{0} t^{\prime}} \mathcal{U}^{-1}\left(t^{\prime}\right)\left[F\left(t^{\prime}\right)+V f\left(t^{\prime}\right)-\varphi\left(t^{\prime}\right) \mathcal{U}^{-1} \Sigma_{3} P_{d} f\left(t^{\prime}\right)\right] d t^{\prime}\right\|_{H^{k_{0}}} \leq \\
& C\left(\sum_{\left|\lambda^{0} \cdot \mu\right|>m-\omega_{0}}\left\|z^{\mu}\right\|_{L^{2}\left(t_{1}, t_{2}\right)}+\left\|R_{1}\right\|_{L_{t}^{1}\left(\left[t_{1}, t_{2}\right], H_{x}^{k_{0}}\right)}+\left\|R_{2}\right\|_{L_{t}^{2}\left(\left[t_{1}, t_{2}\right], H_{x}^{k_{0}, s}\right)}+\|f\|_{L_{t}^{2}\left(\left[t_{1}, t_{2}\right], H_{x}^{k_{0},-\tau_{0}}\right)}\right) .
\end{aligned}
$$

Since the latter has limit 0 as $t_{1} \rightarrow+\infty$, there exists $f_{+}^{\prime} \in H^{k_{0}}$ such that

$$
\lim _{t \rightarrow+\infty}\left\|\mathcal{U}^{-1}(t) f(t)-e^{-\mathrm{i} \mathcal{H}_{0} t} f_{+}^{\prime}\right\|_{H^{k_{0}}}=0 .
$$

From $\mathcal{H}_{0}=D_{m}-\omega_{0} \Sigma_{3}$ and $\mathcal{U}^{-1}(t)=e^{\mathrm{i} \Sigma_{3} \int_{0}^{t} \varphi\left(t^{\prime}\right) d t^{\prime}}$ we have for $\theta(t)=-t \omega_{0}+\int_{0}^{t} \varphi\left(t^{\prime}\right) d t^{\prime}$

$$
\lim _{t \rightarrow+\infty}\left\|e^{\mathrm{i} \theta(t) \Sigma_{3}} f(t)-e^{-\mathrm{i} t D_{m}} f_{+}\right\|_{H^{k_{0}}}=0 .
$$

(10.22) follows from (10.23) if we can prove $\theta(t)=\vartheta(t)-\vartheta(0)+o(1)$ with $o(1) \rightarrow 0$ as $t \rightarrow+\infty$. To prove this claim we substitute $R$ in (2.4) using (4.6) and then replace $(z, f)$ with the last coordinate system obtained from Theorem 9.5. Then we get

$$
\mathrm{i} \dot{f}-\mathcal{H} f-\left(\dot{\vartheta}+\omega_{0}-\sum_{j=2}^{2 N+1} \frac{d}{d t} \Gamma_{0}^{(j)}\right) P_{c}\left(\omega_{0}\right) \Sigma_{3} f=G
$$


where $G$ is a functional with values in $L^{\infty}\left(\mathbb{R}, L_{x}^{1}\right) ; \Gamma_{0}^{(j)}$ are the functions in the exponent of (9.13) for each of the transformations in Theorem 9.5 . Set now

$$
\begin{aligned}
& \chi(t)=\left(\dot{\vartheta}-\omega_{0}-\sum_{j=2}^{2 N+1} \frac{d}{d t} \Gamma_{0}^{(j)}\right)-2\left(\partial_{\|f\|_{2}^{2}} H\right), \\
& \mathbf{G}=-G+\operatorname{rhs} \text { (10.11). }
\end{aligned}
$$

Then taking the difference of the two equations (10.11) and (10.24) we have

$$
\chi(t) f=\chi(t) \Sigma_{3} P_{d}\left(\omega_{0}\right) \Sigma_{3} f+\Sigma_{3} \mathbf{G} .
$$

$\mathbf{G}($ resp.$\chi)$ is a functional from a neighborhood of the origin in $L^{\infty}\left(\mathbb{R}, H^{k_{0}}\left(\mathbb{R}^{3}\right)\right)$ to $L^{\infty}\left(\mathbb{R}, L^{1}\left(\mathbb{R}^{3}\right)\right)$ $\left(\right.$ resp . $\left.L^{\infty}(\mathbb{R})\right)$. If $\chi\left(t_{0}\right) \neq 0$ for a given solution, we can find solutions for which $f_{n}(t, x)$ such that $f_{n}\left(t_{0}, \cdot\right) \rightarrow f\left(t_{0}, \cdot\right)$ in $H^{k_{0}}\left(\mathbb{R}^{3}\right),\left\|f_{n}\left(t_{0}\right)\right\|_{L^{1}\left(\mathbb{R}^{3}\right)} \nearrow \infty, \mathbf{G}_{n}\left(t_{0}\right) \rightarrow \mathbf{G}\left(t_{0}\right)$ and $\chi_{n}\left(t_{0}\right) \rightarrow \chi_{0}\left(t_{0}\right)$. This yields a contradiction. So $\chi \equiv 0$ and $\mathbf{G}=0$. This implies $\dot{\vartheta}-\omega_{0}-\sum_{j=2}^{2 N+1} \frac{d}{d t} \Gamma_{0}^{(j)}=2 \partial_{\|f\|_{2}^{2}} H$. This and the last inequality in (9.14) yield the claim $\theta(t)=\vartheta(t)-\vartheta(0)+o(1)$.

Step (ii). In the proof of Theorem 10.1 consists in introducing the variable

$$
g=f+Y, \quad Y:=\sum_{\left|\lambda^{0} \cdot(\mu-\nu)\right|>m-\omega_{0}} z^{\mu} \bar{z}^{\nu} R_{\mathcal{H}}^{+}\left(\lambda^{0} \cdot(\mu-\nu)\right) H_{\mu \nu}^{0} .
$$

Substituting the new variable $g$ in (10.11), the first line on the rhs of (10.11) cancels out. We have

$$
\begin{aligned}
& \mathrm{i} \dot{g}-\mathcal{H} g-2 \partial_{\|f\|_{2}^{2}} H P_{c}\left(\omega_{0}\right) \Sigma_{3} g=\text { second line of }(10.11)+ \\
& 2 \partial_{\|f\|_{2}^{2}} H P_{c}\left(\omega_{0}\right) \Sigma_{3} Y+\sum_{k=1}^{n}\left[\partial_{z_{k}} Y \partial_{\bar{z}_{k}}(Z+\mathcal{R})-\partial_{\bar{z}_{k}} Y \partial_{z_{k}}(Z+\mathcal{R})\right] .
\end{aligned}
$$

Lemma 10.7. For $\epsilon$ sufficiently small, $\tau_{1}>1$ and $C_{0}=C_{0}(\mathcal{H})$ a fixed constant, we have

$$
\|g\|_{L_{t}^{2}\left([0, T], L_{x}^{\left.2,-\tau_{1}\right)}\right.} \leq C_{0} \epsilon+O\left(\epsilon^{2}\right) .
$$

Proof. Set $F=\left(\right.$ second line of (10.11) $\left.-\varphi(t)\left[\Sigma_{3}, P_{d}\right] g\right)$. Then, proceeding as in (10.18), we have

$$
\begin{aligned}
& \|g\|_{L_{t}^{2} L_{x}^{2,-\tau_{1}}} \leq\left\|e^{-\mathrm{i} t \mathcal{H}_{0}} Y(0)\right\|_{L_{t}^{2} L_{x}^{2,-\tau_{1}}}+\left\|e^{-\mathrm{i} t \mathcal{H}_{0}} f(0)\right\|_{L_{t}^{2} L_{x}^{2,-\tau_{1}}}+C\|F\|_{L_{t}^{1} H_{x}^{k}+L_{t}^{2} H_{x}^{k, \tau_{1}}} \\
& +\| \int_{0}^{t} e^{\mathrm{i}\left(t^{\prime}-t\right) \mathcal{H}_{0}} \text { second line of }\left(\underline{10.25)}\left(t^{\prime}\right) d t^{\prime} \|_{L_{t}^{2} L_{x}^{2,-\tau_{1}}}\right. \\
& +\left\|V_{1}-\varphi(t) \Sigma_{3} P_{d} V_{2}^{-1}\right\|_{L_{t}^{\infty} B\left(L_{x}^{2}, L_{x}^{2,-\tau}\right)}\left\|V_{2} g(t)\right\|_{L_{t x}^{2}} .
\end{aligned}
$$

We have $\left\|e^{-\mathrm{i} t \mathcal{H}_{0}} f(0)\right\|_{L_{t}^{2} L_{x}^{2,-\tau_{1}}} \lesssim\|f(0)\|_{L_{t x}^{2}} \lesssim \epsilon$. We have by Lemma 5.8

$$
\left\|e^{-\mathrm{i} t \mathcal{H}_{0}} Y(0)\right\|_{L_{t}^{2} L_{x}^{2,-\tau_{1}}} \leq C \sum_{\left|\lambda^{0} \cdot(\mu-\nu)\right|>m-\omega_{0}} \epsilon^{|\mu+\nu|} .
$$

We have $\|$ second line of (10.11) $\|_{L_{t}^{1} L_{x}^{2}+L_{t}^{2} L_{x}^{2, \tau_{1}}} \leq O\left(\epsilon^{2}\right)$. Similarly $\left\|\varphi(t)\left[\Sigma_{3}, P_{d}\right] g\right\|_{L_{t}^{2} L_{x}^{2,-\tau_{1}}} \leq C \epsilon\|g\|_{L_{t}^{2} L_{x}^{2, \tau_{1}}}$. Hence $\|F\|_{L_{t}^{1} L_{x}^{2} \cap L_{t}^{2} L_{x}^{2, \tau_{1}}} \leq C \epsilon\|g\|_{L_{t}^{2} L_{x}^{2,-\tau_{1}}}+O\left(\epsilon^{2}\right)$. Now we sketch a bound for the second line of (10.26).

$$
\begin{aligned}
& \sum_{\left|\lambda^{0} \cdot(\mu-\nu)\right|>m-\omega_{0}}\left\|\int_{0}^{t} e^{\mathrm{i}\left(t^{\prime}-t\right) \mathcal{H}_{0}} \partial_{\|f\|_{2}^{2}} H\left(t^{\prime}\right) z^{\mu}\left(t^{\prime}\right) \bar{z}^{\nu}\left(t^{\prime}\right) P_{c}\left(\omega_{0}\right) R_{\mathcal{H}^{*}}^{+}\left(\lambda^{0} \cdot(\mu-\nu)\right) \Sigma_{3} H_{\mu \nu}^{0} d t^{\prime}\right\|_{L_{t}^{2} L_{x}^{2,-\tau_{1}}} \\
& \leq \sum_{\left|\lambda^{0} \cdot(\mu-\nu)\right|>m-\omega_{0}}\left\|\int_{0}^{t}\left\langle t-t^{\prime}\right\rangle^{-\frac{3}{2}}\left|\partial_{\|f\|_{2}^{2}} H\left(t^{\prime}\right) z^{\mu}\left(t^{\prime}\right) \bar{z}^{\nu}\left(t^{\prime}\right)\right| d t^{\prime}\right\|_{L_{t}^{2}} \lesssim C_{2} \epsilon^{2},
\end{aligned}
$$

where we used Lemma 5.8 with $\mathcal{H}$ replaced by $\mathcal{H}^{*}$. Of the other contributions to the second line of (10.26) we focus on the main ones. Specifically we consider for $\mu_{j} \neq 0$

$$
\left\|\int_{0}^{t} e^{\mathrm{i}\left(t^{\prime}-t\right) \mathcal{H}_{0}} P_{c}\left(\omega_{0}\right) \frac{z^{\mu} \bar{z}^{\nu}}{z_{j}} \partial_{\bar{z}_{j}} Z_{0} R_{\mathcal{H}}^{+}\left(\lambda^{0} \cdot(\mu-\nu)\right) H_{\mu \nu}^{0} d t^{\prime}\right\|_{L_{t}^{2} L_{x}^{2,-\tau_{1}}} \leq C\left\|\frac{z^{\mu} \bar{z}^{\nu}}{z_{j}} \partial_{\bar{z}_{j}} Z_{0}\right\|_{L_{t}^{2}}
$$

for $\lambda\left(\omega_{0}\right) \cdot(\mu-\nu)>m-\omega_{0}$. We need to show

$$
\left\|\frac{z^{\mu} \bar{z}^{\nu}}{z_{j}} \partial_{\bar{z}_{j}} Z_{0}\right\|_{L_{t}^{2}}=O\left(\epsilon^{2}\right)
$$


Let $z^{\alpha} \bar{z}^{\beta}$ be a generic monomial of $Z_{0}$. Then $\partial_{\bar{z}_{j}}\left(z^{\alpha} \bar{z}^{\beta}\right)=\beta_{j} \frac{z^{\alpha} \bar{z}^{\beta}}{\bar{z}_{j}}$, with the nontrivial case for $\beta_{j} \neq 0$. By Definition 9.1 we have $\lambda\left(\omega_{0}\right) \cdot(\alpha-\beta)=0$. (H:11) can be applied and implies $|\alpha|=|\beta| \geq 2$. Thus in particular one has

$$
\lambda\left(\omega_{0}\right) \cdot \alpha \geq \lambda_{j}\left(\omega_{0}\right) \Rightarrow \lambda\left(\omega_{0}\right) \cdot(\mu+\alpha)-\lambda_{j}\left(\omega_{0}\right)>m-\omega_{0} .
$$

So the following holds:

$$
\left\|\frac{z^{\mu} \bar{z}^{\nu}}{z_{j}} \frac{z^{\alpha} \bar{z}^{\beta}}{\bar{z}_{j}}\right\|_{L_{t}^{2}} \leq\left\|\frac{z^{\nu} z^{\beta}}{\bar{z}_{j}}\right\|_{L_{t}^{\infty}}\left\|\frac{z^{\mu} z^{\alpha}}{z_{j}}\right\|_{L_{t}^{2}} \leq C C_{2} C_{3} \epsilon^{|\nu|+|\beta|} \leq C C_{2} C_{3} \epsilon^{2} .
$$

We conclude that the second line in (10.26) is $O\left(\epsilon^{2}\right)$. The estimates omitted are easier than (10.27) and (10.28). $\left\|V_{2} g\right\|_{L_{t x}^{2}}$ can be bounded as in Lemma 10.4.

\section{The Fermi gOlden Rule}

Step (iii). We proceed as in [26]. We recall Remark 9.2, In particular we will only consider finite sums $|\mu+\nu|<2 N+3$. We will have $\lambda_{j}^{0}=\lambda_{j}\left(\omega_{0}\right)$ and $\lambda_{j}=\lambda_{j}\left(\|f\|_{2}^{2}\right)$ as in Section $9.1\left|\lambda_{j}^{0}-\lambda_{j}\right| \lesssim C_{1}^{2} \epsilon^{2}$ by (10.6), so in the sequel we can assume that $\lambda^{0}$ satisfies the same inequalities of $\lambda$. Set $R_{\mu \nu}^{+}=R_{\mathcal{H}}^{+}\left(\lambda^{0} \cdot(\mu-\nu)\right)$. We substitute (10.11) in $\mathrm{i} \dot{z}_{j}=\frac{\partial}{\partial \bar{z}_{j}} H^{(r)}$ obtaining

$$
\begin{aligned}
& \mathrm{i} \dot{z}_{j}=\partial_{\bar{z}_{j}}\left(H_{2}+Z_{0}\right)+\sum_{|\lambda \cdot(\mu-\nu)|>m-\omega_{0}} \nu_{j} \frac{z^{\mu} \bar{z}^{\nu}}{\bar{z}_{j}}\left\langle g, \mathrm{i} \beta \alpha_{2} \Sigma_{1} \Sigma_{3} H_{\mu \nu}\right\rangle+\partial_{\bar{z}_{j}} \mathcal{R} \\
& -\sum_{\substack{|\lambda \cdot(\alpha-\beta)|>m-\omega_{0} \\
|\lambda \cdot(\mu-\nu)|>m-\omega_{0}}} \nu_{j} \frac{z^{\mu+\alpha} \bar{z}^{\nu+\beta}}{\bar{z}_{j}}\left\langle R_{\alpha \beta}^{+} H_{\alpha \beta}^{0}, \mathrm{i} \beta \alpha_{2} \Sigma_{1} \Sigma_{3} H_{\mu \nu}\right\rangle .
\end{aligned}
$$

We rewrite this as

$$
\begin{aligned}
& \mathrm{i} \dot{z}_{j}=\partial_{\bar{z}_{j}}\left(H_{2}+Z_{0}\right)+\mathcal{E}_{j} \\
& -\sum \quad \begin{array}{l}
\lambda \cdot \beta>m-\omega_{0} \\
\lambda \cdot \nu>m-\omega_{0}
\end{array} \quad \nu_{j} \frac{\bar{z}^{\nu+\beta}}{\bar{z}_{j}}\left\langle R_{0 \beta}^{+} H_{0 \beta}^{0}, \mathrm{i} \beta \alpha_{2} \Sigma_{1} \Sigma_{3} H_{0 \nu}^{0}\right\rangle \\
& \lambda \cdot \beta-\lambda_{k}<m-\omega_{0} \forall k \text { s.t. } \beta_{k} \neq 0 \\
& -\sum \begin{array}{c}
\lambda \cdot \alpha>m-\omega_{0} \\
\lambda>\nu>m-\omega_{0}
\end{array} \nu_{j} \frac{z^{\alpha} \bar{z}^{\nu}}{\bar{z}_{j}}\left\langle R_{\alpha 0}^{+} H_{\alpha 0}^{0}, \mathrm{i} \beta \alpha_{2} \Sigma_{1} \Sigma_{3} H_{0 \nu}^{0}\right\rangle .
\end{aligned}
$$

Here the elements in (11.3) will be eliminated through a new change of variables. $\mathcal{E}_{j}$ is a reminder term defined by

$$
\mathcal{E}_{j}:=\operatorname{rhs}(11.1)-(11.3)-(11.4)
$$

Set

$$
\begin{aligned}
& \zeta_{j}=z_{j}-\sum_{(\beta, \nu) \text { as in [11.3) }} \frac{\nu_{j}}{\lambda^{0} \cdot(\beta+\nu)} \frac{\bar{z}^{\nu+\beta}}{\bar{z}_{j}}\left\langle R_{0 \beta}^{+} H_{0 \beta}^{0}, \mathrm{i} \beta \alpha_{2} \Sigma_{1} \Sigma_{3} H_{0 \nu}^{0}\right\rangle \\
& +\sum_{(\alpha, \nu) \text { as in }[11.4)} \frac{\nu_{j}}{\lambda^{0} \cdot(\alpha-\nu)} \frac{z^{\alpha} \bar{z}^{\nu}}{\bar{z}_{j}}\left\langle R_{\alpha 0}^{+} H_{\alpha 0}^{0}, \mathrm{i} \beta \alpha_{2} \Sigma_{1} \Sigma_{3} H_{0 \nu}^{0}\right\rangle
\end{aligned}
$$

Notice that in (11.5), by $\lambda \cdot \nu>\omega_{0}-m$, we have $|\nu|>1$. Then by (10.7)

$$
\begin{aligned}
& \|\zeta-z\|_{L_{t}^{2}} \leq C \epsilon \sum_{\substack{\lambda \cdot \alpha>m-\omega_{0} \\
\lambda \cdot \alpha-\lambda_{k}<m-\omega_{0} \forall k \text { s.t. } \alpha_{k} \neq 0}}\left\|z^{\alpha}\right\|_{L_{t}^{2}} \leq C C_{2} M \epsilon^{2} \\
& \|\zeta-z\|_{L_{t}^{\infty}} \leq C^{3} \epsilon^{3}
\end{aligned}
$$

with $C$ the constant in (10.5) and $M$ the number of terms in the rhs. In the new variables (11.2) is of the form 


$$
\begin{aligned}
& \mathrm{i} \dot{\zeta}_{j}=\partial_{\bar{\zeta}_{j}} H_{2}(\zeta, f)+\partial_{\bar{\zeta}_{j}} Z_{0}(\zeta, f)+\mathcal{D}_{j} \\
& -\sum_{\substack{\lambda^{0} \cdot \alpha=\lambda^{0} \cdot \nu>m-\omega_{0} \\
\lambda \cdot \alpha-\lambda_{k}<m-\omega_{0} \forall k \text { s.t. } \alpha_{k} \neq 0 \\
\lambda \cdot \nu-\lambda_{k}<m-\omega_{0} \forall k \text { s.t. } \nu_{k} \neq 0}} \nu_{j} \frac{\zeta^{\alpha} \bar{\zeta}^{\nu}}{\bar{\zeta}_{j}}\left\langle R_{\alpha 0}^{+} H_{\alpha 0}^{0}, \mathrm{i} \beta \alpha_{2} \Sigma_{1} \Sigma_{3} H_{0 \nu}^{0}\right\rangle .
\end{aligned}
$$

From these equations by $\sum_{j} \lambda_{j}^{0}\left(\bar{\zeta}_{j} \partial_{\bar{\zeta}_{j}}\left(H_{2}+Z_{0}\right)-\zeta_{j} \partial_{\zeta_{j}}\left(H_{2}+Z_{0}\right)\right)=0$ we get

$$
\begin{aligned}
& \partial_{t} \sum_{j=1}^{n} \lambda_{j}^{0}\left|\zeta_{j}\right|^{2}=2 \sum_{j=1}^{n} \lambda_{j}^{0} \Im\left(\mathcal{D}_{j} \bar{\zeta}_{j}\right)- \\
& -2 \sum_{(\alpha, \nu) \text { as in }} \lambda^{0} \cdot \nu \Im\left(\zeta^{\alpha} \bar{\zeta}^{\nu}\left\langle R_{\alpha 0}^{+} H_{\alpha 0}^{0}, \mathrm{i} \beta \alpha_{2} \Sigma_{1} \Sigma_{3} H_{0 \nu}^{0}\right\rangle\right) .
\end{aligned}
$$

We have the following lemma, whose proof (we skip) is similar to Lemma 4.7 [25]:

Lemma 11.1. Assume inequalities (10.7). Then for a fixed constant $c_{0}$ we have

$$
\sum_{j}\left\|\mathcal{D}_{j} \bar{\zeta}_{j}\right\|_{L^{1}[0, T]} \leq\left(1+C_{2}\right) c_{0} \epsilon^{2}
$$

For the sum in the second line of (11.8) we get

$$
\begin{aligned}
& 2 \sum_{r>m-\omega_{0}} r \Im\left\langle R_{\mathcal{H}}^{+}(r) \sum_{\lambda^{0} \cdot \alpha=r} \zeta^{\alpha} H_{\alpha 0}^{0}, \mathrm{i} \beta \alpha_{2} \Sigma_{1} \Sigma_{3} \sum_{\lambda^{0} \cdot \nu=r}\left(\zeta^{\nu}\right)^{*} H_{0 \nu}^{0}\right\rangle= \\
& 2 \sum_{r>m-\omega_{0}} r \Im\left\langle R_{\mathcal{H}}^{+}(r) \sum_{\lambda^{0} \cdot \alpha=r} \zeta^{\alpha} H_{\alpha 0}^{0}, \Sigma_{3}\left[\sum_{\lambda^{0} \cdot \alpha=r} \zeta^{\alpha} H_{\alpha 0}^{0}\right]^{*}\right\rangle=2 \sum_{r>m-\omega_{0}} r \Im\left\langle R_{\mathcal{H}}^{+}(r) \mathbf{H}_{r}, \Sigma_{3} \mathbf{H}_{r}^{*}\right\rangle,
\end{aligned}
$$

where $\mathbf{H}_{r}:=\sum_{\lambda^{0} \cdot \alpha=r} \zeta^{\alpha} H_{\alpha 0}^{0}$ and where we have used $\mathrm{i} \beta \alpha_{2} \Sigma_{1} \Sigma_{3} H_{\mu \nu}^{0}=-\Sigma_{3} \mathrm{i} \beta \alpha_{2} \Sigma_{1} H_{\nu \mu}^{0}=\Sigma_{3} \mathrm{i} \beta \alpha_{2} C H_{\nu \mu}^{0}=$ $\Sigma_{3}\left(H_{\nu \mu}^{0}\right)^{*}$ by (9.10).

Lemma 11.2. Consider $\mathbf{H}_{r}$ in (11.10). Assume $m-\omega_{0}<r<m+\omega_{0}$. Then

$$
\Im\left\langle R_{\mathcal{H}}^{+}(r) \mathbf{H}_{r}, \Sigma_{3} \mathbf{H}_{r}^{*}\right\rangle \geq 0 .
$$

If we assume (H:3), in particular if $m / 3<\omega_{0}<m$, then (11.11) holds for all $\mathbf{H}_{r}$ in (11.10).

Proof. We proceed as in Lemma 10.5 [26]. Set $\mathbf{F}_{r}=\mathcal{Z}_{+} \mathbf{H}_{r}$, where for $\mathcal{Z}_{+}$with $\omega=\omega_{0}$, see Theorem A.3 in the Appendix. Set $\mathbf{F}_{r}=\left(\begin{array}{l}a \\ b\end{array}\right)$. Then

$$
\begin{aligned}
& \Im\left\langle R_{\mathcal{H}}^{+}(r) \mathbf{H}_{r}, \Sigma_{3} \mathbf{H}_{r}^{*}\right\rangle=\lim _{\varepsilon \searrow 0} \Im\left\langle R_{\mathcal{H}}(r+\mathrm{i} \varepsilon) \mathbf{H}_{r}, \Sigma_{3} \mathbf{H}_{r}^{*}\right\rangle=\lim _{\varepsilon \searrow 0} \Im\left\langle R_{\mathcal{H}_{\omega_{0}, 0}}(r+\mathrm{i} \varepsilon) \mathbf{F}_{r}, \Sigma_{3} \mathbf{F}_{r}^{*}\right\rangle \\
& =\lim _{\varepsilon \searrow 0} \Im\left\langle R_{D_{m}}(r+\omega+\mathrm{i} \varepsilon) a, a^{*}\right\rangle-\lim _{\varepsilon \searrow 0} \Im\left\langle R_{D_{m}}(r-\omega+\mathrm{i} \varepsilon) b, b^{*}\right\rangle \\
& =\frac{1}{2} \lim _{\varepsilon \searrow 0} \varepsilon\left\|R_{D_{m}}(r+\omega+\mathrm{i} \varepsilon) a\right\|_{L^{2}}^{2}-\Im\left\langle R_{D_{m}}(r-\omega) b, b^{*}\right\rangle=\frac{1}{2} \lim _{\varepsilon \searrow 0} \varepsilon\left\|R_{D_{m}}(r+\omega+\mathrm{i} \varepsilon) a\right\|_{L^{2}}^{2} \geq 0 .
\end{aligned}
$$

Here we exploited that $a, b \in L^{2}\left(\mathbb{R}^{3}\right)$, that $r-\omega<m$ and so $R_{D_{m}}(r-\omega)$ is a well defined selfadjoint operator in $L^{2}\left(\mathbb{R}^{3}\right)$, that $R_{D_{m}}(z)-R_{D_{m}}\left(z^{*}\right)=2 \mathrm{i} R_{D_{m}}(z) R_{D_{m}}\left(z^{*}\right) \Im z$ and that $R_{D_{m}}\left(z^{*}\right)=\left(R_{D_{m}}(z)\right)^{*}$.

Let us consider $r=\lambda \cdot \mu$ with $\mu \in \mathbb{N}_{0}^{n}, \lambda \cdot \mu>m-\omega_{0}$ and $\lambda \cdot \mu-\lambda_{k}<m-\omega_{0}$ for all $k$ s.t. $\mu_{k} \neq 0$. Suppose $\lambda \cdot \mu>m+\omega_{0}$. Then we get $m-\omega_{0}+\lambda_{k}>m+\omega_{0} \Rightarrow \lambda_{k}>2 \omega_{0}$. Let $N_{k} \in \mathbb{N}$ such that $N_{k} \lambda_{k}<m-\omega_{0}<\left(N_{k}+1\right) \lambda_{k}$ as in (H:9) Then $\left(2 N_{k}+1\right) \omega_{0}<m$. So, if we assume as in (H:3) that $\omega_{0}>m / 3$, we obtain $\lambda \cdot \mu<m+\omega_{0}$. This shows that the assumption $\lambda \cdot \mu>m+\omega_{0}$ is absurd.

Remark 11.3. Notice that to get the conclusions of Lemma 11.2 we can ease the constraint $3 \omega>m$ to $\left(2 N_{k}+1\right) \omega>m$ for all $k=1, \ldots, n$.

Now we will assume the following hypothesis. 
(H:12') We assume that for some fixed constant $C>0$, for any vector $\zeta \in \mathbb{C}^{n}$ we have:

$$
\begin{aligned}
& \sum_{(\alpha, \nu) \text { as in } 11.7} \lambda^{0} \cdot \nu \Im\left(\zeta^{\alpha} \bar{\zeta}^{\nu}\left\langle R_{\alpha 0}^{+} H_{\alpha 0}^{0}, \mathrm{i} \beta \alpha_{2} \Sigma_{1} \Sigma_{3} H_{0 \nu}^{0}\right\rangle\right) \\
& \geq C \sum_{\substack{\lambda^{0} \cdot \alpha>m-\omega_{0} \\
\lambda^{0} \cdot \alpha-\lambda_{k}^{0}<m-\omega_{0} \forall k \text { s.t. } \alpha_{k} \neq 0}}\left|\zeta^{\alpha}\right|^{2} .
\end{aligned}
$$

Remark 11.4. By Lemma 11.2 we have lhs(11.12) $\geq 0$. It is likely then that (H:12') is true generically in the class of non linearities we consider. But we do not try to prove this point.

By (H:12') we have

$$
2 \sum_{j=1}^{n} \lambda_{j}^{0} \Im\left(\mathcal{D}_{j} \bar{\zeta}_{j}\right) \gtrsim \partial_{t} \sum_{j=1}^{n} \lambda_{j}^{0}\left|\zeta_{j}\right|^{2}+\sum_{\substack{\lambda^{0} \cdot \alpha>m-\omega_{0} \\ \lambda^{0} \cdot \alpha-\lambda_{k}^{0}<m-\omega_{0} \forall k \text { s.t. } \alpha_{k} \neq 0}}\left|\zeta^{\alpha}\right|^{2} .
$$

Then, for $t \in[0, T]$ and assuming Lemma 11.1 we have

$$
\sum_{j=1}^{n} \lambda_{j}^{0}\left|\zeta_{j}(t)\right|^{2}+\sum_{\alpha \text { as in (11.13) }}\left\|\zeta^{\alpha}\right\|_{L^{2}(0, t)}^{2} \lesssim \epsilon^{2}+C_{2} \epsilon^{2} .
$$

By (11.6) this implies $\left\|z^{\alpha}\right\|_{L^{2}(0, t)}^{2} \lesssim \epsilon^{2}+C_{2} \epsilon^{2}$ for all the above multi indexes. So, from $\left\|z^{\alpha}\right\|_{L^{2}(0, t)}^{2} \lesssim C_{2}^{2} \epsilon^{2}$ we conclude $\left\|z^{\alpha}\right\|_{L^{2}(0, t)}^{2} \lesssim C_{2} \epsilon^{2}$.

Note that as the condition $|\lambda \cdot(\mu-\nu)|>m-\omega$ implies that $|\mu+\nu| \geq 2$, (11.1) implies that $\dot{z}$ is integrable so that it has a limit at infinity which is necessarily 0 . This yields Theorem 10.1 and completes the proof of Theorem 1.3

11.1. Proof of Theorem 8.2. We only sketch the proof, which is similar to that of Theorem 8.1, For a particular solution satisfying the hypotheses of Theorem 8.2 we need to prove the conclusions of Theorem 10.1. The argument is exactly the same of Section 10.1 until we reach subsection 11, that is the task of estimating $z$. Instead of (11.7) we have

$$
\begin{aligned}
& \mathrm{i} \dot{\zeta}_{j}=\varepsilon_{j} \partial_{\bar{\zeta}_{j}} H_{2}(\zeta, f)+\varepsilon_{j} \partial_{\bar{\zeta}_{j}} Z_{0}(\zeta, f)+\varepsilon_{j} \mathcal{D}_{j} \\
& -\varepsilon_{j} \sum_{(\alpha, \nu) \text { as in } 11.7} \nu_{j} \frac{\zeta^{\alpha} \bar{\zeta}^{\nu}}{\bar{\zeta}_{j}}\left\langle R_{\alpha 0}^{+} H_{\alpha 0}^{0}, \mathrm{i} \beta \alpha_{2} \Sigma_{1} \Sigma_{3} H_{0 \nu}^{0}\right\rangle .
\end{aligned}
$$

From these equations by $\sum_{j} \lambda_{j}^{0}\left(\bar{\zeta}_{j} \partial_{\bar{\zeta}_{j}}\left(H_{2}+Z_{0}\right)-\zeta_{j} \partial_{\zeta_{j}}\left(H_{2}+Z_{0}\right)\right)=0$ we get

$$
\begin{aligned}
& \partial_{t} \sum_{j=1}^{n} \varepsilon_{j} \lambda_{j}^{0}\left|\zeta_{j}\right|^{2}=2 \sum_{j=1}^{n} \lambda_{j}^{0} \Im\left(\mathcal{D}_{j} \bar{\zeta}_{j}\right)- \\
& -2 \sum_{(\alpha, \nu) \text { as in } 11.7} \lambda^{0} \cdot \nu \Im\left(\zeta^{\alpha} \bar{\zeta}^{\nu}\left\langle R_{\alpha 0}^{+} H_{\alpha 0}^{0}, \mathrm{i} \beta \alpha_{2} \Sigma_{1} \Sigma_{3} H_{0 \nu}^{0}\right\rangle\right) .
\end{aligned}
$$

The estimate of the reminder term in Lemma 11.1 continues to hold. The last line of (11.14) is negative by (11.10). We assume it is strictly negative and that in particular (11.12) holds. Then we get

$$
\sum_{\alpha \text { as in }[11.13)}\left|\zeta^{\alpha}\right|^{2} \lesssim-\partial_{t} \sum_{j=1}^{n} \varepsilon_{j} \lambda_{j}^{0}\left|\zeta_{j}\right|^{2}+2 \sum_{j=1}^{n} \lambda_{j}^{0} \Im\left(\mathcal{D}_{j} \bar{\zeta}_{j}\right) .
$$

When we integrate in $(0, t)$ for $t \leq T$ we get

$$
\sum_{\alpha \text { as in }[11.13)}\left\|\zeta^{\alpha}\right\|_{L^{2}(0, t)}^{2} \lesssim \epsilon^{2}+C_{2} \epsilon^{2} .
$$

In the rhs we have used the hypothesis $|z(t)| \leq \epsilon$ for all $t \geq 0$ to bound the first summation in the rhs of (11.15). This yields Theorem 8.2 . 
11.2. Proof of Theorem 8.4. Also here we just sketch the proof, which is similar to [24]. The proof is by contradiction. If the statement of Theorem 8.4 is wrong, then for $|z(0)|+\|f(0)\|_{H^{k_{0}}} \leq \delta$ with $\delta>0$ sufficiently small, we can assume $|z(t)| \leq \epsilon$ for all $t \geq 0$ for any preassigned $\epsilon>0$. This implies that we can apply Theorem 8.2. When get

$$
\sum_{\alpha \text { as in }}\left\|\zeta^{\alpha}\right\|_{L^{2}(0, t)}^{213} \lesssim \sum_{j=1}^{n} \varepsilon_{j} \lambda_{j}^{0}\left(\left|\zeta_{j}(0)\right|^{2}-\left|\zeta_{j}(t)\right|^{2}\right)+2 \int_{0}^{t} \sum_{j=1}^{n} \lambda_{j}^{0} \Im\left(\mathcal{D}_{j} \bar{\zeta}_{j}\right) .
$$

Suppose $\varepsilon_{j_{0}}=-1$. Then take initial datum $z_{j}(0)=0$ for $j \neq j_{0}, z_{j_{0}}=\delta$ and $f(0)=0$. By $f(0)=0$ and Lemma 10.4 for $\psi(0)=0$ we get for $t \in \mathbb{R}^{+}$

$$
\begin{gathered}
\|f\|_{L_{t}^{p} B_{q, 2}^{k_{0}-\frac{3}{p}} \cap L_{t}^{2} H_{x}^{k_{0},-\tau_{0}} \cap L_{t}^{2} L_{x}^{\infty}} \lesssim \mathcal{Y}^{2}+\left\|R_{1}\right\|_{L_{t}^{1} H_{x}^{k_{0}}}+\left\|R_{2}\right\|_{L_{t}^{2} H_{x}^{k_{0}, \tau_{0}}} \\
\mathcal{Y}^{2}:=\sum_{\alpha \text { as in } \overline{11.13}}\left\|z^{\alpha}\right\|_{L_{t}^{2} .}
\end{gathered}
$$

Similarly

$$
\|g\|_{L_{t}^{2} L_{x}^{2,-\tau_{1}}} \lesssim \delta^{2}+\epsilon \mathcal{Y}^{2}+\left\|R_{1}\right\|_{L_{t}^{1} H_{x}^{k_{0}}}+\left\|R_{2}\right\|_{L_{t}^{2} H_{x}^{k_{0}, \tau_{0}}} .
$$

Then, proceeding as in [24, 25] one improves the rhs in (11.9). Indeed, see Lemma 4.9 [25], we have

$$
\sum_{j}\left\|\mathcal{D}_{j} \bar{\zeta}_{j}\right\|_{L^{1}\left(\mathbb{R}^{+}\right)} \leq C \mathcal{Y}\|g\|_{L_{t}^{2} H_{x}^{-4,-s}}+C \epsilon \mathcal{Y}^{2}+C\left\|R_{1}\right\|_{L_{t}^{1} H_{x}^{k_{0}}}+C\left\|R_{2}\right\|_{L_{t}^{2} H_{x}^{k_{0}, \tau_{0}}} .
$$

Then, one can see that $\left\|R_{1}\right\|_{L_{t}^{1} H_{x}^{k_{0}}}+\left\|R_{2}\right\|_{L_{t}^{2} H_{x}^{k_{0}, \tau_{1}}} \lesssim o(1) \delta$, going through Lemma 10.3, where $o(1) \rightarrow 0$ as $\delta \rightarrow 0$. Then from (11.16) we get $\mathcal{Y}^{2} \lesssim-\delta+o(1) \delta$, which is absurd.

\section{Appendix A. Resolvent estimates And Wave operators}

Lemma A.1. We assume $\left(\mathbf{H : 1 )}\right.$ and $\left(\mathbf{H : 6 )}\left(\mathbf{H : 8 )}\right.\right.$, Then for any $\tau>1$ there exists a constant $C_{1}=$ $C_{1}(\tau, \omega)$ upper semicontinuous in $\omega$ s.t. for any $u_{0}(x) \in L^{2}\left(\mathbb{R}^{3}, \mathbb{C}^{8}\right)$ and any $\varepsilon>0$ we have

$$
\left\|\langle x\rangle^{-\tau} R_{\mathcal{H}_{\omega}}(\lambda \pm \mathrm{i} \varepsilon) P_{c}\left(\mathcal{H}_{\omega}\right) u_{0}\right\|_{L_{\lambda, x}^{2}\left(\mathbb{R}^{4}\right)} \leq C_{1}\left\|P_{c}\left(\mathcal{H}_{\omega}\right) u_{0}\right\|_{L^{2}\left(\mathbb{R}^{3}\right)} .
$$

Proof. Notice that by Lemma 5.1 for any $\tau>1$, any $u_{0}(x) \in L^{2}\left(\mathbb{R}^{3}, \mathbb{C}^{8}\right)$ and any $\varepsilon>0$ we have

$$
\left\|\langle x\rangle^{-\tau} R_{\mathcal{H}_{\omega, 0}}(\lambda \pm \mathrm{i} \varepsilon) u_{0}\right\|_{L_{\lambda, x}^{2}\left(\mathbb{R}^{4}\right)} \leq C(\tau)\left\|u_{0}\right\|_{L^{2}\left(\mathbb{R}^{3}\right)} .
$$

Let $u_{0}=P_{c}\left(\mathcal{H}_{\omega}\right) u_{0}, A(x)=\langle x\rangle^{-\tau}$ and $B(x) \in \mathcal{S}\left(\mathbb{R}^{3}, B\left(\mathbb{C}^{8}, \mathbb{C}^{8}\right)\right)$ s.t. $B^{*} A=V_{\omega}$. Then

$$
A R_{\mathcal{H}_{\omega}}(z) u_{0}=\left(1+A R_{\mathcal{H}_{\omega, 0}}(z) B^{*}\right)^{-1} A R_{\mathcal{H}_{\omega, 0}}(z) u_{0} .
$$

The following operators preserve $\mathbf{X}: A, B^{*}, R_{\mathcal{H}_{\omega}}(z)$ and $R_{\mathcal{H}_{\omega 0}}(z)$. Pick $\delta_{0}>0$ sufficiently small so that by (H:6) for any $\lambda_{j}(\omega) \in \sigma_{d}\left(\mathcal{H}_{\omega}\right)$ we have $\left|\lambda_{j}(\omega)\right|<m-\omega-\delta_{0}$. Then by (A.2) and (A.3), Lemma A.1 is a consequence of the Lemma A.2 below.

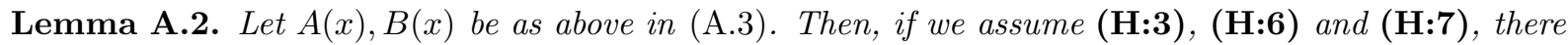
exists a constant $C_{2}=C_{2}(\tau, \omega)$ upper semicontinuous in $\omega$ such that for any $\varepsilon>0$ we have

$$
\sup _{\lambda \in\left(\mathbb{R} \backslash\left[-m+\omega+\delta_{0}, m-\omega-\delta_{0}\right]\right)}\left\|\left(1+A R_{\mathcal{H}_{\omega, 0}}(\lambda \pm \mathrm{i} \varepsilon) B^{*}\right)^{-1}\right\|_{B(\mathbf{X}, \mathbf{X})} \leq C_{2} .
$$

For any $\tau>1$ the limit $R_{\mathcal{H}_{\omega}}^{+}(\lambda)=\lim _{\varepsilon \searrow 0} R_{\mathcal{H}_{\omega}}(\lambda \pm \mathrm{i} \varepsilon)$ exist in $B\left(H_{x}^{1, \tau} \cap \mathbf{X}, L_{x}^{2,-\tau}\right)$ and the convergence is uniform for $\lambda$ in compact sets.

Proof. First of all we prove (A.4) in low energies. We want to prove

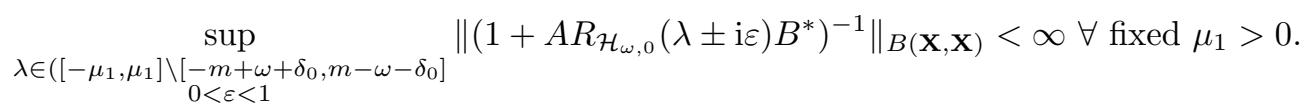

We know: $z \rightarrow A R_{\mathcal{H}_{\omega, 0}}(z) B^{*}$ is a holomorphic map with domain $\mathbb{C} \backslash \mathbb{R}$ and values in $B(\mathbf{X}, \mathbf{X})$; for all $z \in \mathbb{C} \backslash \mathbb{R},\left(1+A R_{\mathcal{H}_{\omega, 0}}(\lambda \pm \mathrm{i} \varepsilon) B^{*}\right)^{-1}$ is defined . Furthermore, $\lim _{\varepsilon \succ 0} A R_{\mathcal{H}_{\omega, 0}}(\lambda \pm \mathrm{i} \varepsilon) B^{*}$, by (ii) Lemma 5.1 exists in $B(\mathbf{X}, \mathbf{X})$ and the convergence is uniform for $\lambda$ in compact sets. Then we apply 
Lemma 7.5 [7] and conclude that, outside closed sets $\Gamma^{ \pm} \subset \mathbb{R}$ with 0 Lebesgue measure in $\mathbb{R}$, the map $z \rightarrow\left(1+A R_{\mathcal{H}_{\omega, 0}}(z) B^{*}\right)^{-1}$ extends in a continuous map defined in $\{z: \Im z>0\} \cup\left(\mathbb{R} \backslash \Gamma^{+}\right)$(resp. $\{z: \Im z<$ $\left.0\} \cup\left(\mathbb{R} \backslash \Gamma^{-}\right)\right)$with values in $B(\mathbf{X}, \mathbf{X})$. Given $\lambda \in \Gamma^{+}$there exists $\psi \in \mathbf{X} \backslash\{0\}$ with $\psi=-A R_{\mathcal{H}_{\omega, 0}}^{+}(\lambda) B^{*} \psi$. But then, by standard arguments $u:=R_{\mathcal{H}_{\omega, 0}}^{+}(\lambda) B^{*} \psi \in L^{2,-\tau}\left(\mathbb{R}^{3}, \mathbb{C}^{8}\right)$ is a nonzero solution of (1.2). By (H7)-(H8) we have $u \in L^{2}\left(\mathbb{R}^{3}, \mathbb{C}^{8}\right)$. Furthermore $\psi \in \mathbf{X}$ implies $u \in \mathbf{X}$. But by (H6) no such $u \in \mathbf{X}$ can exist. So the intersection of $\Gamma^{+}$with $\mathbb{R} \backslash\left(-m+\omega+\delta_{0}, m-\omega-\delta_{0}\right)$ is empty. A similar argument shows that the intersection of $\Gamma^{-}$with $\mathbb{R} \backslash\left(-m+\omega+\delta_{0}, m-\omega-\delta_{0}\right)$ is empty.

Having considered the low energy case (A.5), we consider for $\mu_{1}$ any fixed large real number:

$$
\sup _{|\lambda| \geq \mu_{1}}\left\|\left(1+A R_{\mathcal{H}_{\omega, 0}}^{ \pm}(\lambda) B^{*}\right)^{-1}\right\|_{B\left(L_{x}^{2}, L_{x}^{2}\right)} \leq C_{3}
$$

For definiteness we will consider $\lambda \geq \mu_{1}$. We consider the expansion $\sum_{\ell=0}^{\infty}\left(A R_{\mathcal{H}_{\omega, 0}}^{ \pm}(\lambda) B^{*}\right)^{\ell}$. We start now the implementation of the high energy argument in [30. We have

$$
\begin{aligned}
& R_{\mathcal{H}_{\omega, 0}}^{ \pm}(\lambda)=\left(\begin{array}{cc}
R_{D_{m}}^{ \pm}(\lambda+\omega) & 0 \\
0 & R_{D_{m}}^{ \pm}(\lambda-\omega)
\end{array}\right)=R_{0}^{ \pm}(\lambda) \mathcal{A}(\lambda, \nabla) \\
& R_{0}^{ \pm}(\lambda):=\left(\begin{array}{cc}
R_{-\Delta+m^{2}}^{ \pm}\left((\lambda+\omega)^{2}\right) I_{2} & 0 \\
0 & R_{-\Delta+m^{2}}^{ \pm}\left((\lambda-\omega)^{2}\right) I_{2}
\end{array}\right) \\
& \mathcal{A}(\lambda, \nabla):=\left(\begin{array}{cc}
\mathcal{A}_{1}(\lambda, \nabla) & 0 \\
0 & \mathcal{A}_{2}(\lambda, \nabla)
\end{array}\right), \quad \mathcal{A}_{j}(\lambda, \nabla):=\left(\begin{array}{cc}
\lambda-(-1)^{j} \omega+m & -\mathrm{i} \sigma \cdot \nabla \\
-\mathrm{i} \sigma \cdot \nabla & \lambda-(-1)^{j} \omega-m
\end{array}\right) .
\end{aligned}
$$

For definiteness let us consider $R_{\mathcal{H}_{\omega, 0}}^{+}$. Let now $\chi_{0}, \psi_{0} \in C_{0}^{\infty}(\mathbb{R})$ by cutoffs supported near 0 and let $\chi_{1}:=1-\chi_{0}$ and $\psi_{1}:=1-\psi_{0}$. We can choose them so that

$$
\chi_{1}(|x-y|)=\left(\psi_{0}(|x|) \psi_{1}(|y|)+\psi_{1}(|x|) \psi_{0}(|y|)+\psi_{1}(|x|) \psi_{1}(|y|)\right) \chi_{1}(|x-y|)
$$

We split for a fixed large number $M_{0}>0$

$$
\begin{aligned}
& R_{-\Delta+m^{2}}^{+}\left(\left(\lambda-(-1)^{j} \omega\right)^{2}, x, y\right)=\sum_{\ell=0}^{1} R_{\ell j}(\lambda, x, y), \\
& R_{\ell j}(\lambda, x, y):=\frac{e^{\mathrm{i} \sqrt{\left(\lambda-(-1)^{j} \omega\right)^{2}+m^{2}}|x-y|}}{4 \pi|x-y|} \chi_{\ell}\left(\frac{|x-y|}{M_{0}}\right) .
\end{aligned}
$$

We have a decomposition $R_{\mathcal{H}_{\omega, 0}}^{+}=R_{\mathcal{H}_{\omega, 0}}^{0+}+R_{\mathcal{H}_{\omega, 0}}^{1+}$ with kernels $R_{\mathcal{H}_{\omega, 0}}^{j+}=\chi_{j}\left(\frac{|\cdot|}{M_{0}}\right) R_{\mathcal{H}_{\omega, 0}}^{+}$. By (A.9)- A.10) and by [1] there exists $c_{M_{0}}$ with $\lim _{M_{0} \rightarrow+\infty} c_{M_{0}}=0$ s.t.

$$
\sup _{\lambda \in \mathbb{R}}\left\|A R_{\mathcal{H}_{\omega, 0}}^{1+}(\lambda) B^{*}\right\|_{B\left(L_{x}^{2}, L_{x}^{2}\right)} \leq c_{M_{0}} .
$$

By $\left\|A R_{\mathcal{H}_{\omega, 0}}^{ \pm}(\lambda) B^{*}\right\|_{B\left(L_{x}^{2}, L_{x}^{2}\right)} \leq C$, for fixed $C^{\prime}$ we have

$$
\left\|A R_{\mathcal{H}_{\omega, 0}}^{0 \pm}(\lambda) B^{*}\right\|_{B\left(L_{x}^{2}, L_{x}^{2}\right)} \leq C^{\prime} .
$$

We have

$$
R_{0 j}(\lambda, x, y)=\lambda R_{-\Delta}^{+}\left(\sqrt{\left(1-(-1)^{j} \frac{\omega}{\lambda}\right)^{2}+\frac{m^{2}}{\lambda^{2}}}, \lambda x, \lambda y\right) \chi_{0}\left(\frac{|x-y|}{M_{0}}\right) .
$$

Key to showing that (A.6) follows directly from [30 is the observation that we can write

$$
R_{-\Delta}^{+}\left(\sqrt{\left(1-(-1)^{j} \frac{\omega}{\lambda}\right)^{2}+\frac{m^{2}}{\lambda^{2}}}, x, y\right) \chi_{0}\left(\frac{|x-y|}{\lambda M_{0}}\right)=\frac{e^{\mathrm{i}|x-y|}}{|x-y|} a_{\lambda, j}(|x-y|)+\frac{b_{\lambda, j}(|x-y|)}{|x-y|},
$$

with

$$
\begin{aligned}
& \left|a_{\lambda, j}^{(k)}(r)\right| \leq C\left(M_{0}, k\right) r^{-k} \quad \forall k \geq 0, \quad a_{\lambda, j}^{(k)}(r)=0 \quad \forall 0<r<1 \\
& \left|b_{\lambda, j}^{(k)}(r)\right| \leq C\left(M_{0}, k\right) \quad \forall k \geq 0, \quad b_{\lambda, j}^{(k)}(r)=0 \quad \forall r>2 .
\end{aligned}
$$


Notice that A.14 - A.15) are formulas of the same type of (3.2)-(3.4) [30. As a consequence for any fixed small $\delta_{0}>0$ there are $\ell_{0}=\ell\left(\delta_{0}\right)$ and $\mu_{1}=\mu_{1}\left(\delta_{0}\right)$ such that for $\lambda \geq \mu_{1}$ we have

$$
\left\|\left(A \chi_{0} R_{\mathcal{H}_{\omega, 0}^{0}}^{+}(\lambda) B^{*}\right)^{\ell_{0}}\right\|_{B\left(L_{x}^{2}, L_{x}^{2}\right)} \leq \delta_{0} .
$$

For $\ell$ large and $\delta_{0} \leq c_{M_{0}}$, by (A.11), (A.12) and (A.16) we get

$$
\left\|\left(A R_{\mathcal{H}_{\omega, 0}^{0+}}^{0+}(\lambda) B^{*}+A R_{\mathcal{H}_{\omega, 0}}^{1+}(\lambda) B^{*}\right)^{\ell}\right\|_{B\left(L_{x}^{2}, L_{x}^{2}\right)} \leq 2^{\ell}\left(2 C^{\prime}\right)^{\ell} c_{M_{0}}^{\frac{\ell}{\ell_{0}}}
$$

For $c_{M_{0}}$ sufficiently small, (A.17) implies (A.6).

We finish with the following corollary of Lemma A.1.

Theorem A.3. Assume the hypotheses of Lemma A.1. Pick the A, $B^{*}$ of (A.3). Then there are isomorphisms $\mathcal{W}_{ \pm}: \mathbf{X} \rightarrow \mathbf{X}_{c}\left(\mathcal{H}_{\omega}\right)$ and $\mathcal{Z}_{ \pm}: \mathbf{X}_{c}\left(\mathcal{H}_{\omega}\right) \rightarrow \mathbf{X}$, inverses of each other, defined as follows: for $u \in \mathbf{X}$, $v \in \mathbf{X}_{c}\left(\mathcal{H}_{\omega}\right)$,

$$
\begin{aligned}
& \left\langle\mathcal{W}_{ \pm} u, v^{*}\right\rangle=\left\langle u, v^{*}\right\rangle \mp \lim _{\epsilon \rightarrow 0^{+}} \frac{1}{2 \pi \mathrm{i}} \int_{\mathbb{R}}\left\langle A R_{\mathcal{H}_{\omega, 0}}(\lambda \pm \mathrm{i} \epsilon) u,\left(B R_{\mathcal{H}_{\omega}^{*}}(\lambda \pm \mathrm{i} \epsilon) v\right)^{*}\right\rangle d \lambda \\
& \left\langle\mathcal{Z}_{ \pm} v, u^{*}\right\rangle=\left\langle v, u^{*}\right\rangle \pm \lim _{\epsilon \rightarrow 0^{+}} \frac{1}{2 \pi \mathrm{i}} \int_{\mathbb{R}}\left\langle A R_{\mathcal{H}_{\omega}}(\lambda \pm \mathrm{i} \epsilon) v,\left(B R_{\mathcal{H}_{\omega, 0}^{*}}(\lambda \pm \mathrm{i} \epsilon) u\right)^{*}\right\rangle d \lambda .
\end{aligned}
$$

$\mathcal{W}_{ \pm}\left(\right.$resp. $\left.\mathcal{Z}_{ \pm}\right)$define isomorphisms $H^{k}\left(\mathbb{R}^{3}, \mathbb{C}^{8}\right) \cap \mathbf{X} \rightarrow P_{c}\left(\mathcal{H}_{\omega}\right) H^{k}\left(\mathbb{R}^{3}, \mathbb{C}^{8}\right)$ (resp. and viceversa) for all $k$. We also have

$$
\begin{aligned}
& \mathcal{W}_{ \pm} u=\lim _{t \rightarrow \pm \infty} e^{\mathrm{i} t \mathcal{H}_{\omega}} e^{-\mathrm{i} t \mathcal{H}_{\omega, 0}} u \text { for all } u \in \mathbf{X} \\
& \mathcal{Z}_{ \pm} v=\lim _{t \rightarrow \pm \infty} e^{\mathrm{i} t \mathcal{H}_{\omega, 0}} e^{-\mathrm{i} t \mathcal{H}_{\omega}} v \text { for all } v \in \mathbf{X}_{c}\left(\mathcal{H}_{\omega}\right)
\end{aligned}
$$

Proof. The proof follows by Lemma A.1 by means of the argument for Theorem 1.5 [40. (A.19) follows by Theorem 3.9 [40.

\section{REFERENCES}

[1] S.Agmon. Spectral properties of Schrödinger operators and scattering theory. An. Sc. N. Pisa, 2(2):151-218, 1975.

[2] D.Bambusi and S.Cuccagna. On dispersion of small energy solutions of the nonlinear klein gordon equation with a potential, Amer. Math. Jour., 133(1):1421-1468, 2011.

[3] M.Balabane, T.Cazenave, A.Douady, and F.Merle. Existence of excited states for a nonlinear Dirac field. Com. Math. Phys., 119(1):153-176, 1988.

[4] M.Balabane, T.Cazenave, and L.Vázquez. Existence of standing waves for Dirac fields with singular nonlinearities. Com. Math. Phys., 133(1):53-74, 1990.

[5] M.Beceanu. A centre-stable manifold for the focussing cubic NLS in $\mathbb{R}^{1+3}$. Com. Math. Phys., 280(1):145-205, 2008.

[6] G. Berkolaiko and A. Comech. On spectral stability of solitary waves of nonlinear dirac equation on a line, arXiv:0910.0917

[7] A.-M.Berthier. Spectral theory and wave operators for the Schrödinger equation, vol. 71 of Res. N. in Math. Pitman, Boston, Mass., 1982.

[8] A.-M.Berthier and V.Georgescu. On the point spectrum of Dirac operators. J. Fun. Anal., 71(2):309-338, 1987.

[9] N.Boussaid. Stable directions for small nonlinear Dirac standing waves. Com. Math. Phys., 268(3):757-817, 2006.

[10] N.Boussaid. On the asymptotic stability of small nonlinear Dirac standing waves in a resonant case. SIAM $J$. Math. Anal., 40(4):1621-1670, 2008.

[11] N.Bournaveas. Local well-posedness for a nonlinear Dirac equation in spaces of almost critical dimension. Discr. Cont. Dyn. Syst., 20(3):605-616, 2008.

[12] V.Buslaev and G.Perel'man. Scattering for the nonlinear Schrödinger equation: states that are close to a soliton. Algebra i Analiz, 4(6):63-102, 1992.

[13] V.Buslaev and G.Perel'man. On the stability of solitary waves for nonlinear Schrödinger equations. In Nonlinear evolution equations, vol. 164 Am. Math. Soc. Tran. Ser. 2, pages 75-98, AMS, Providence, RI, 1995.

[14] P.Brenner. On space-time means and everywhere defined scattering operators for nonlinear Klein-Gordon equations. Math. Zeitschrift, 186(3):383-391, 1984.

[15] T.Cazenave and P.-L.Lions. Orbital stability of standing waves for some nonlinear Schrödinger equations. Com. Math. Phys., 85(4):549-561, 1982.

[16] T.Cazenave and L.Vázquez. Existence of localized solutions for a classical nonlinear Dirac field. Com. Math. Phys., 105(1):35-47, 1986.

[17] Shu-Ming Chang, Stephen Gustafson, Kenji Nakanishi, and Tai-Peng Tsai. Spectra of linearized operators for NLS solitary waves. SIAM J. Math. Anal., 39(4):1070-1111, 2007/08. 
[18] M.Christ and A.Kiselev. Maximal functions associated to filtrations. J. Fun. Anal., 179(2):409-425, 2001.

[19] M.Chugunova and D.Pelinovsky. Block-diagonalization of the symmetric first-order coupled-mode system. SIAM J. Appl. Dyn. Syst., 5(1):66-83, 2006.

[20] A.Comech. On the meaning of the Vakhitov-Kolokolov stability criterion for the nonlinear Dirac equation, arXiv:1107.1763 2011.

[21] M.Chugunova and D.Pelinovsky. Block-diagonalization of the symmetric first-order coupled-mode system. SIAM J. Appl. Dyn. Syst., 5(1):66-83 (electronic), 2006.

[22] S.Cuccagna. Stabilization of solutions to nonlinear Schrödinger equations. Com. Pure Appl. Math., 54(9):1110$1145,2001$.

[23] S.Cuccagna. On asymptotic stability in energy space of ground states of NLS in 1D. J. Diff. Eq., 245(3):653-691, 2008.

[24] S.Cuccagna. On instability of excited states of the nonlinear Schrödinger equation. Phys. D, 238(1):38-54, 2009.

[25] S.Cuccagna. On scattering of small energy solutions of non autonomous hamiltonian nonlinear schrödinger equations, J. Diff. Eq., 250(5):2347-2371, 2011.

[26] S.Cuccagna. The hamiltonian structure of the nonlinear Schrödinger equation and the asymptotic stability of its ground states, Com. Math. Phys., 305:279-331, 2011.

[27] S.Cuccagna. On asymptotic stability of moving ground states of the nonlinear Schrodinger equation, arXiv:1107.4954 3, 2011, to appear Tran.AMS.

[28] S.Cuccagna and T.Mizumachi. On asymptotic stability in energy space of ground states for nonlinear Schrödinger equations. Com. Math. Phys., 284(1):51-77, 2008.

[29] S.Cuccagna, D.Pelinovsky, and V. Vougalter. Spectra of positive and negative energies in the linearized NLS problem. Com. Pure Appl. Math., 58(1):1-29, 2005.

[30] M.B.Erdoğan, M.Goldberg, and W.Schlag. Strichartz and smoothing estimates for Schrödinger operators with almost critical magnetic potentials in three and higher dimensions. Forum Math., 21(4):687-722, 2009.

[31] M.J.Esteban, M.Lewin, and E.Séré. Variational methods in relativistic quantum mechanics. Bull. Amer. Math. Soc. (N.S.), 45(4):535-593, 2008.

[32] M.J. Esteban and É. Séré. Stationary states of the nonlinear Dirac equation: a variational approach. Com. Math. Phys., 171(2):323-350, 1995.

[33] Zhou Gang, I.M.Sigal, Relaxation of Solitons in Nonlinear Schrödinger Equations with Potential. dvances in Math., 216: 443-490, 2007.

[34] V.Georgescu and M.Măntoiu. On the spectral theory of singular Dirac type Hamiltonians. J. Oper. Th., 46(2):289-321, 2001.

[35] M.Grillakis, J.Shatah and W.Strauss. Stability theory of solitary waves in the presence of symmetry. I. J. Fun. Anal., 74(1):160-197, 1987.

[36] M. Grillakis, J.Shatah and W. Strauss. Stability theory of solitary waves in the presence of symmetry. II. J. Fun. Anal., 94(2):308-348, 1990.

[37] Meijiao Guan. Solitary wave solutions for the nonlinear Dirac equations, arXiv:0812.2273 2008.

[38] P.D.Hislop. Exponential decay of two-body eigenfunctions: a review. In Proceedings of the Symposium on Mathematical Physics and Quantum Field Theory (Berkeley, CA, 1999), Conf. 4 Electron. J. Differ. Equ. Conf., pages 265-288 (electronic), San Marcos, TX, 2000. Southwest Texas State Univ.

[39] A.Iftimovici and M.Măntoiu. Limiting absorption principle at critical values for the Dirac operator. Lett. Math. Phys., 49(3):235-243, 1999.

[40] T.Kato. Wave operators and similarity for some non-selfadjoint operators. Math. Ann., 162:258-279, 1965/1966.

[41] A.Komech and A.Komech. On global attraction to quantum stationary states. Diracequation with mean field interaction, February 2010.

[42] F.Merle. Sur la non-existence de solutions positives d'équations elliptiques surlinéaires. C. R. Acad. Sci. Paris Sér. I Math., 306(6):313-316, 1988.

[43] Y.Martel and F.Merle. Asymptotic stability of solitons of the gKdV equations with general nonlinearity. Math. Ann., 341(2):391-427, 2008.

[44] K.Nakanishi and W.Schlag. Global dynamics above the ground state energy for the cubic nls equation in 3d, July 2010.

[45] H.Ounaies. Perturbation method for a class of nonlinear Dirac equations. Diff. Int. Eq., 13(4-6):707-720, 2000.

[46] D.Pelinovsky and A.Stefanov. Asymptotic stability of small gap solitons in the nonlinear dirac equations, arXiv:1008.4514, 2010.

[47] A.F.Ranada. Classical nonlinear Dirac field models of extended particles. Quantum theory, groups, fields and particles, 271-291, 1982.

[48] M.Reed and B.Simon. Methods of modern mathematical physics. IV. Analysis of operators. Academic Press [Harcourt Brace Jovanovich Publishers], New York, 1978.

[49] J.Shatah. Stable standing waves of nonlinear Klein-Gordon equations. Com. Math. Phys., 91:313-327, 1983.

[50] J.Shatah and W.Strauss. Instability of nonlinear bound states. Com. Math. Phys., 100:173-190, 1985.

[51] C.D.Sogge. Lectures on nonlinear wave equations. International Press Boston, 1995.

[52] M.Soler. Classical, stable, nonlinear spinor field with positive rest energy. Phys. Rev. D, 1(10):2766-2769, 1970.

[53] H.Smith and C.Sogge. Global Strichartz estimates for nontrapping perturbations of the Laplacian. Com. Part. Diff. Eq., 25(11-12):2171-2183, 2000.

[54] Y.Saitō and T.Umeda. The zero modes and zero resonances of massless Dirac operators. Hokkaido Math. J., $37(2): 363-388,2008$.

[55] W.Strauss and L. Vázquez. Stability under dilations of nonlinear spinor fields. Phys. Rev. D (3), 34(2):641-643, 1986. 
[56] A.Soffer and M.I.Weinstein. Multichannel nonlinear scattering theory for nonintegrable equations. Com. Math. Phys., 133(1):116-146, 1990.

[57] A.Soffer and M.I.Weinstein. Multichannel nonlinear scattering for nonintegrable equations. II. The case of anisotropic potentials and data. J. Diff. Eq., 98(2):376-390, 1992.

[58] M.Taylor. Partial differential equations., volumes 115-117 of Applied Mathematical Sciences. Springer-Verlag, New York, 1996.

[59] B.Thaller. The Dirac equation. Texts and Monographs in Physics. Springer-Verlag, Berlin, 1992.

[60] T.P.Tsai, H.T.Yau, Asymptotic dynamics of nonlinear Schrödinger equations: resonance dominated and radiation dominated solutions. Com. Pure Appl. Math. 55153-216, 2002.

[61] M.I.Weinstein. Modulational stability of ground states of nonlinear Schrödinger equations. SIAM J. Math. Anal., 16(3):472-491, 1985.

[62] M.I.Weinstein. Lyapunov stability of ground states of nonlinear dispersive evolution equations. Com. Pure Appl. Math., 39(1):51-67, 1986.

Laboratoire de mathématiques, UFR Sciences et Technicques, Université de Franche-Comté, 16, route de Gray, 25030 Besançon, France

E-mail Address: nabile.boussaid@univ-fcomte.fr

Department of Mathematics and Geosciences, University of Trieste, Via Valerio 12/1 Trieste, 34127 Italy.

E-mail Address: scuccagna@units.it 